\title{
Axiomatic formulations of modified gravity theories with nonlinear dispersion relations and Finsler-Lagrange-Hamilton geometry
}

\author{
Laurenţiu Bubuianu ${ }^{1,2, a}$, Sergiu I. Vacaru ${ }^{3,4,5, b}$ \\ 1 TVR Iaşi, 33 Lascăr Catargi Street, Iasi, Romania \\ ${ }^{2}$ University Apollonia, 2 Muzicii Street, 700107 Iasi, Romania \\ 3140 Morehampton Rd, Donnybrook, Dublin 04, D04 N2C0, Ireland \\ ${ }^{4}$ Physics Department, California State University Fresno, Fresno, CA 93740, USA \\ ${ }^{5}$ Project IDEI, University “Al. I. Cuza” Iaşi, Iasi, Romania
}

Received: 26 October 2018 / Accepted: 8 November 2018 / Published online: 23 November 2018

(C) The Author(s) 2018

\begin{abstract}
We develop an axiomatic geometric approach and provide an unconventional review of modified/nonlinear gravity theories, MGTs, with modified dispersion relations, MDRs, encoding Lorentz invariance violations, LIVs, classical and quantum random effects, anisotropies etc. There are studied Lorentz-Finsler like theories elaborated as extensions of general relativity, GR, and quantum gravity, QG, models and constructed on (co)tangent Lorentz bundles, i.e. (curved) phase spaces or locally anisotropic spacetimes. An indicator of MDRs is considered as a functional on various type functions depending on phase space coordinates and physical constants. It determines respective generating functions and fundamental physical objects (generalized metrics, connections and nonholonomic frame structures) for relativistic models of Finsler, Lagrange and/or Hamilton spaces. We show that there are canonical almost symplectic differential forms and adapted (non)linear connections which allow us to formulate equivalent almost KählerLagrange/-Hamilton geometries. This way, it is possible to unify geometrically various classes of (non)commutative MGTs with locally anisotropic gravitational, scalar, nonAbelian gauge field, and Higgs interactions. We elaborate on theories with Lagrangian densities containing massive graviton terms and bi-connection and bi-metric modifications which can be modelled as Finsler-Lagrange-Hamilton geometries. An example of short-range locally anisotropic gravity on (co)tangent Lorentz bundles is analysed. We conclude that a large class of such MGTs admits a self-consistent causal axiomatic formulation which is similar to GR but
\end{abstract}

\footnotetext{
a e-mail: laurentiu.bubuianu@tvr.ro

be-mails: sergiu.vacaru@gmail.com; sergiuvacaru@mail.fresnostate.edu
}

involving generalized (non)linear connections, Finsler metrics and adapted frames on phase spaces. Such extensions of the standard model of particle physics and gravity offer a comprehensive guide to classical formulation of MGTs with MDRs, their quantization, applications in modern astrophysics and cosmology, and search for observable phenomena and experimental verifications.

\section{Contents}

1 Introduction ................ 2

2 Phase spaces with MDRs and Finsler-LagrangeHamilton geometry . . . . . . . . . . . . . 5

2.1 Motivations and assumptions . . . . . . . . 5

2.2 Canonical N-connections, metrics and almost symplectic structures . . . . . . . . . 9

2.2.1 MDR, Hamilton-Lagrange generating functions and $\mathrm{N}$-connections . . . . . . . 9

2.2.2 Locally anisotropic metrics on (co)tangent Lorentz bundles . . . . . . . . . . . . . 11

2.3 Almost Kähler Lagrange-Hamilton structures and MDRs . . . . . . . . . . . 12

2.3.1 Canonical almost complex structures and Neijenhuis fields . . . . . . . . . 12

2.3.2 Canonical almost symplectic structures determined by MDRs . . . . . . . . . . . 13

2.4 Lagrange-Hamilton connections and curvatures 14 2.4.1 Distinguished connections, $\mathrm{N}$-adapted distortions and curvatures . . . . . . . . 15

2.4.2 The Ricci and Einstein d-tensors . . . . . 16

2.4.3 Physically important (Filsler like) dconnections on (co)tangent bundles . . . 17 \section{年} 2 2 ?

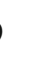

9


2.4.4 Distortion tensors for connections and curvature and Ricci tensors . . . . . . . . 18

2.4.5 On Akbar-Zadeh definition of the Ricci tensor for Finsler like spaces . . . . . . . 20

3 Geometric and physical principles for gravity theories on (co)tangent bundles . . . . . . . . . . . . 20

3.1 Principles for extending GR to Finsler-Lagrange-

Hamilton gravity theories . . . . . . . . . . 21

3.1.1 Modified equivalence principles . . . . . 21

3.1.2 Generalized Mach principles . . . . . . . 22

3.1.3 Principle of general covariance and equivalent geometrization of MGTs . . . . . . 23

3.1.4 Principles of analogy of $\mathrm{N}$-adapted operators defined by d-metrics and d-connections 24

3.2 Lagrange densities and energy-momentum tensors on (co)tangent Lorentz bundles . . . . . . 25

3.2.1 Scalar fields on (co)tangent Lorentz bundles 25

3.2.2 Lagrange densities for Einstein-YangMills-Higgs systems with MDRs . . . . 25

3.2.3 Actions for minimal MDR-extensions of GR and YMH theories . . . . . . . . 26

3.2.4 Actions for MGTs with massive gravitons, bi-metric structures and MDRs . . . 28

3.2.5 Lagrange densities for short-range locally anisotropic gravity with MDRs and LIV . 30

3.2.6 The equations of motion and nonholonomic conservation laws for (effective) sources ............. 31

4 Modified field equations with MDRs and LagrangeHamilton gravity . . . . . . . . . . . . . 32

4.1 Generalized Einstein equations on nonholonomic (co)tangent Lorentz bundles . . . . . . . 32

4.1.1 Nonholonomic Einstein equations with general (effective) sources . . . . . . . 33

4.1.2 Gravitational equations on Lorentz cotangent bundles ........... . 33

4.2 Modified Einstein equations for pseudo Lagrange-

Hamilton spaces . . . . . . . . . . . . . 33

4.2.1 Einstein-Finsler-Lagrange gravity . . . . 34

4.2.2 Einstein-Hamilton gravity . . . . . . . . 34

4.3 MDRs and the Einstein-Yang-Mills-Higgs equa-

tions ................ . . 35

4.3.1 EYMH systems on pseudo Lagrange spaces 35

4.3.2 EYMH systems on pseudo Hamilton spaces 35

4.4 Massive and bi-metric MGTs as Lagrange-

Hamilton geometries . . . . . . . . . . . 35

4.4.1 Generalized Einstein equations with massive graviton on tangent Lorentz bundles . 35

4.4.2 Massive gravitational equations on cotangent Lorentz bundles . . . . . . . . . . 36

4.5 Short-range locally anisotropic gravity . . . . . 36

4.5.1 Field equations for short-range gravity with MDRs . . . . . . . . . . 36
4.5.2 Field equations for short-range co-gravity with MDRs . . . . . . . . . . 36

4.6 Towards axiomatic formulation of MGTs with MDRs on (co)tangent bundles . . . . . . . . . 36

4.6.1 Assumptions on metrics, nonlinear quadratic line elements and d-metrics . . . . . . . 37

4.6.2 Geometric and physical principles for Finsler-Lagrange-Hamilton gravity extensions .............. 38

4.6.3 Conventions on Lagrangians and energymomentum tensors on (co)tangent bundles 39

4.6.4 Principles and main theorems for modified field equations with MDRs . . . . . 40

5 Conclusions, achievements and perspectives . . . . 41

5.1 On geometric models of MGTs with MDRs and generalized relativistic Finsler spaces . . . . . . 41

5.2 The physical picture of Finsler-Lagrange-Hamilton gravity theories . . . . . . . . . . . 44 43

5.3 What has been achieved in mathematical and theoretical physics with Finsler methods? . . . 44

5.4 What is missing in the scheme and further perspectives . . . . . . . . . . . . 45

A Two important corollaries for N-adapted formulas . 46

A.1 Curvatures and torsions of d-connections on (co)tangent bundles . . . . . . . . . . . 46

A.2 The coefficients of canonical Lagrange and Hamilton d-connections . . . . . . . . . . . . 46

References ................ . 47

\section{Introduction}

Over the past two decades, the literature on classical and quantum gravity, QG, and accelerating cosmology has been increased substantially involving spacetime models with non-Riemannian geometries and modified gravity theories, MGTs. Various approaches to commutative and noncommutative theories, when Planck-scale features and deformed classical and quantum symmetries are described by modified dispersion relations, MDRs, and possible (local) Lorentz invariance violations, LIVs, have been developed in $[4,30$, $32,51,71,77,78,90,95,96,142,147-150,155,189,192]$ and references therein. Such works have provided a series of important results on QG and string phenomenology and physics of relativistic particles propagating and interacting in effective media with MDRs. There were elaborated models for doubly-special and/or deformed-special relativity; theories with LIVs, deformed curved phase spacetimes with (non)commutative and/or (non)associative variables etc. A subclass of MGTs was formulated as models of (generalized) Finsler geometry, see Refs. [15,49,50,79,83, 108, 116, $153,161,169,175,183,185,194,208]$ for reviews and critical remarks. 
The Einstein gravity theory (i.e. general relativity, GR) has to be modified into a theory on (co)tangent bundles to Lorentz manifolds if MDRs are stated, for instance, as small deformations of standard quadratic dispersion relations in special relativity theory, SRT, and (locally) in GR, by some indicator functions depending on velocity/momentum variables. Such constructions were elaborated for generalized spinor, gauge and string gravity models with Finsler-Lagrange-Hamilton configurations [133, $134,147,149,150,152]$. There were studied also models on higher order (co)tangent bundles with possible supersymmetric/noncommutative variables and almost symplectic structures. A series of results on MGTs with local anisotropy and Finsler like modifications were reviewed and presented in $[153,161,169,173,185]$. It was shown that such constructions involve MDRs which can be derived for propagation of point mass classical particles and/or models with quantum variables and (non)commutative relations describing quantum fundamental field interactions $[32,59-63,156,157,168$, 174,179,216,217]. Various classes of locally anisotropic (non)commutative/associative classical and quantum field and spacetime theories can be described naturally in terms of nonholonomic variables as some models of commutative and/or noncommutative geometries. For corresponding parameterizations and distributions of geometric/physical objects, we can construct (effective) Lagrange-Finsler, (dual) Hamilton-Cartan, and/or other type phase space and spacetime models. We cite also some results on Hamilton geometry for generalizations of standard physical theories and general relativity, GR, contained in Part I of monograph [161] and papers $[8,208] .{ }^{1}$ Those works contain a number of original results on relativistic models on (co)tangent bun-

\footnotetext{
${ }^{1}$ In our works, we use various terms like pseudo Lagrange/Finsler space/geometry and/or, equivalently, relativistic Lagrange geometry/mechanics. There are also elaborated the concepts of "pseudo, i.e. relativistic" Finsler, Hamilton or other types non-Riemannian geometries. The term pseudo-Lagrange is used for a corresponding Hessian (Lagrange metric) like in pseudo-Euclidean (locally) and/or pseudo-Riemannian geometry. Mathematicians in many cases write semi-Riemannian (instead of pseudo-Riemannian) when such a space is endowed, at least locally and/or effectively, with metric structures of pseudo-Euclidean signature, which in this work are labeled $(+,+,+,-)$, on Lorentz manifolds and $(+,+,+,-;+,+,+,-)$, on (co)tangent Lorentz bundle. This encodes a very important experimental fact that the physical interactions of fields and propagation of particles are described by a (maximal) constant speed of light and possible polarizations in certain effective media. (Pseudo) Finsler-LagrangeHamilton geometries (which will be defined and studied rigorously in next sections) are determined by nonlinear quadratic elements for which the concept of signature cannot be defined in a general form. Nevertheless, we assume and prove that for certain very general conditions and realistic physical models we can always introduce an associated (effective) quadratic form uniquely determined by a fundamental nonlinear quadratic form following certain physically motivated geometric principles. For such effective quadratic forms (metrics), we can consider the concept of signature, classify some small perturbations of physical fields and propagation of particles to be relativistic, or non-relativistic,
}

dles and/or on nonholonomic manifolds, with higher order extensions and, in particular, for constructing new classes exact solutions in Einstein gravity by introducing nonholonomic $2+2$ (co)fibred structures, see $[68,151,158,171$, 182,186,188,198,201,203]. Recently, a model of Hamilton geometry with MDRs was studied in [14].

Planck-scale modifications of dispersion relations are studied in phenomenological particle models, cosmology, and astrophysics. There are some indications that Finsler like generalizations allows us to account for the Planckian structures of relativistic classical and quantum field interactions by extending theories on conventional configuration space. Various ideas and geometric and physical models were analyzed in $[3-7,33,34,37,69,71,77-81,93,94,96$, 119-122,133,134,187,189,192]. In such approaches, the standard-model extensions originate from the idea of spontaneous breaking of the Lorentz and CPT symmetries in the string theory and involved vacuum expectation values of tensor fields with spacetime indices, all resulting in certain models with MDRs and LIVs.

The goal of this work is to formulate a self-consistent geometric approach to MGTs constructed on phase spaces encoding MDRs and LIV when such theories extend GR on (co)tangent Lorentz bundles. We show that such models can be elaborated equivalently as Finsler-Lagrange-Hamilton gravity theories. Applying $\mathrm{N}$-adapted variational and nonholonomic geometric methods, there are proved/formulated generalized Einstein equations. There are derived also dynamical equations and (effective) matter sources for locally anisotropic scalar fields, non-Abelian gauge and Higgs fields and distortions of (non)linear connection structures. This article provides a general theoretical background for a series of future partner papers on exact solutions with Lagrange-Hamilton variables, quantization of such theories, and applications in modern cosmology and astrophysics. Such works will develop the anholonomic frame deformation method, AFDM, for constructing exact solutions in MGTs formulated in explicit Hamilton like variables. Various applications of AFDM and examples of Finsler like generic off-diagonal commutative and/or noncommutative, supersymmetric/brane/string and other types of black ellipsoid/hole, wormhole, solitonic and/or cosmological solutions have been elaborated and reviewed in Refs. $[9,68,69,151,153,158-165,168,169$, 182,183,186,188-190,196,198,199,201-203]. Alternative

\footnotetext{
Footnote 1 continued

ones in an effective media (aether, or vacuum spacetime model). In a number of recent work, the terms "pseudo" and "relativistic" are used for MGTs with local properties described by an associated quadratic form are like in Lorentz geometry. In a more general context, there are considered nonlinear and linear connection structures, nonholonomic constraints and generalized symmetries, which are different from those in (pseudo) Riemannian geometry.
} 
approaches on Finsler gravity theories and attempts to construct physical models and find approximate solutions can be studied in $[25,39,40,53,66,67,84-88,98-100,111,112$, $126,136-139,141]$. It is considered that readers are familiar with standard results on mathematical relativity, geometry of (non)linear connections in fiber bundles, spinor differential geometry, and exact classical solutions in GR (see, for instance, $[48,82,104,213])$. On certain technical topics under consideration, we shall refer to specific monographs and reviews where details and ample biographic information can be found. This article is also oriented to open-minded scholars and young researchers on geometry and physics who are curious about application of advanced geometric methods for solving new extraordinary open problems in modern classical and quantum gravity, cosmology and astrophysics, and standard and nonstandard particle physics.

The paper is organized as follows: In Sect. 2, we formulate a geometric approach to gravity theories with MDRs and LIVs modelled on relativistic Lagrange-Finsler and Hamilton-Cartan phase spaces. There are provided important motivations and main assumptions which are necessary for elaborating such geometric and physical models. There are defined canonical (non)linear connections, metrics and almost symplectic structures determined by MDRs encoding LIVs and possible contributions from QG, massive gravity and bi-metric and bi-connection terms. Corresponding curvature, torsion and nonmetricity tensors (and related Ricci and Einstein tensors, scalar curvatures of various classes of Finsler like connections and their distortions) are constructed in $\mathrm{N}$-adapted forms for (co)tangent Lorentz bundles.

Section 3 is devoted to a study of general principles for formulating MGTs with MDRs on (co)tangent Lorentz bundles. It is proven that such extensions of the Einstein gravity can be performed in canonical forms for Lagrange and Hamilton like variables. There are defined minimal actions and Lagrange densities for Einstein-Yang-Mills-Higgs systems with MDRs and analyzed possible contributions by massive gravitons and theories with bi-metric locally anisotropic structure. Actions and sources are considered for short-range gravity models with LIVs and anisotropic interactions.

Following geometric and $\mathrm{N}$-adapted variational methods, we study (in Sect. 4) possible generalizations of the Einstein equations for theories with MDRs on (co)tangent Lorentz bundles. There are derived gravitational and matter field equations for Lagrange-Hamilton spaces, EYMH systems, massive and bi-metric locally anisotropic MGTs and shortrange phenomenological gravitational theories with LIVs resulting in locally anisotropic configurations. The constructions are provided both in coordinate-free and $\mathrm{N}$-adapted coefficient forms. We speculate on axiomatic approaches to geometrizing MGTs on (co)tangent Lorentz bundles.

In Sect. 5, there are concluded the main results. We speculate on geometrization of MGTs with MDRs and the phys- ical picture of Finsler-Lagrange-Hamilton gravity theories. In brief, we summarize what has been achieved with Finsler modifications of the GR and discuss what is missing. Open problems and further perspectives in study cosmological Einstein-Hamilton and locally anisotropic Einstein-Dirac configurations determined by MDRs, elaborating quantum gravity models on generalized (non)commutative and (non)associative phase spaces are analyzed.

Proofs of theorems are sketched in some forms being accessible both for researchers on mathematical physics and phenomenology of particle physics. We prefer the so-called abstract geometric method for proofs and emphasize the possibility of alternative $\mathrm{N}$-adapted variational approaches. Certain technical details are given in Appendix A. Throughout the main part of this article, there are also provided brief historical comments on "relativistic" Finsler like theories (with effective metrics of local Lorentzian signature) and generalizations of the Einstein gravity with MDRs on Lagrange-Hamilton spaces. We note that a historical review on Finsler geometry and physics and a discussion of conventional 20 main directions of our research activity is presented Appendix B of [208]. ${ }^{2}$ Such surveys of second

\footnotetext{
${ }^{2}$ For reviews on relativistic developments and MGTs, we cite $[8,153$, $161,169,173,175,185,194]$ and references therein. Here we note that for local Euclidean signatures, the main ideas on Lagrange-Hamilton geometries formulated as generalized Finsler spaces on (co)tangent bundles were proposed and studied in a series of works due to J. Kern, M. Matsumoto, S. Ikeda, see [54-58,64,65,75,91,92]. There were elaborated theories on supersymmetric and higher order generalizations, almost Kähler models and applications in particle physics and gravity. Later, similar constructions and some applications in non-relativistic geometric mechanics classical field models were developed in a series of monographs [101-103], see critical remarks in Appendix B of [208]. Those books were based on some hundreds of papers containing as the main author the name Radu Miron (a member of the Romanian Academy of Sciences), who falsified and omitted to stipulate a series of original and important former contributions of a number of researchers from Japan, former USSR, Hungary, Germany, USA, China etc. and his former co-authors like G. Atanasiu, A. Bejancu, V. Oproiu, and tenths others. During last 60 years, more than 250 articles were published (the first papers contained co-authors who "disappeared" in further developments) in "not-accessible" Romanian journals and local preprints. Later those results were re-published by R. Miron (as an individual or first author, and with various modifications) in more than 30 monographs. This can be checked, for instance, tracking the names in MathSciNet. Contributions of Western authors and even from the former USSR were censored in Romania. A series of monographs and collections of works were published by Kluwer, Hadronic Press, Romanian Academy etc. under the names R. Miron and a few other politically and ideologically selected (by communists and Ceauşescu's secret service) co-authors who sworn as doctor-docents an "absolute devote" to Romanian dictator's wife, Elena Ceauşescu. Those works were on developments and mechanical applications of (higher order) Lagrange-FinslerHamilton-Cartan geometries. No matter where those articles and books were published (in Romania, Japan, or in some Western Countries), the content was elaborated in a style of "higher order hidden plagiarism", with falsification of results and politically/ideologically screened teams of authors. Unfortunately, none ideological and communist political lustration of former communist and secrete service leaders, and
} 
author's research (together with his former students and coauthors) performed beginning 1982 in (former) USSR, the R. Moldova, and (later) in Romania and Western Countries, and supported by a number of NATO, DAAD, UNESCO and CERN fellowships and grants, is important because it provides evidences that those results anticipated a series of recent publications on modified Finsler gravity theories and applications in modern cosmology.

\section{Phase spaces with MDRs and Finsler-Lagrange-Hamilton geometry}

This section contains an introduction to the geometry of nonholonomic (co)tangent Lorentz bundles which is used for elaborating relativistic Finsler-Lagrange and HamiltonCartan gravity theories. There are developed a series of concepts and constructions considered in Part I of monograph [161], on relativistic and modified Hamilton spaces, and for MGTs defined by MDRs and LIVs generalizations of GR as generalized Finsler theories. For reviews and critical remarks on Finsler-Lagrange-Hamilton geometry and gravity theories with nonholonomic Lorentzian manifolds and tangent bundles, and applications in modern cosmology and astrophysics, we cite $[8,153,161,169,175,183,185,194]$ and references therein.

\subsection{Motivations and assumptions}

In semi-classical commutative and/or noncommutative models of MGTs and/or QG theories, a MDR can be written locally in a general form

$c^{2} \overrightarrow{\mathbf{p}}^{2}-E^{2}+c^{4} m^{2}=\varpi\left(E, \overrightarrow{\mathbf{p}}, m ; \ell_{P}\right)$.

An indicator of deformations/modifications $\varpi(.$.$) encodes$ in a functional form possible contributions of modified geometric and physical theories and LIVs. Such MDRs can be extended to dependencies on spacetime coordinates $x^{i}=$ $\left(x^{1}, x^{2}, x^{3}, x^{4}=c t\right)$ (one can be introduced extra dimensions) on a standard Lorentz manifold $V$ and/or generalizations with metric-affine structures. In explicit form, certain classes of $\varpi\left(x^{i}, E, \overrightarrow{\mathbf{p}}, m ; \ell_{P}\right)$ are chosen and studied following theoretical/phenomenological arguments, determined experimentally, and/or computed in a generalized classical/quantum theory of gravity and matter filed interactions. The formula (1) transforms into a standard quadratic dispersion relation for a relativistic point particle with mass $m$,

\section{Footnote 2 continued}

administrators in science, was in Romania (like it was, for instance, in Eastern Germany or other former "socialist" Countries). There is not a complete access even to files on the history of physics and mathematics when "ideology and secret service" were deeply involved. energy $E$, and momentum $p_{i}$ (for $i=1,2,3$ ) propagating in a four dimensional, 4-d, flat Minkowski spacetime of signature $(+,+,+,-)$ if $\varpi=0$. It is supposed that certain modifications of the special relativity theory, SRT, and GR could be consequences of some (deformed) modified symmetries, for instance, with (non)commutative deformed Poincaré transforms, quantum groups and interactions, Lorentz invariance violation etc. Such values involve redefinitions of the physical energy-momentum $p_{a}=\left(p_{i}, p_{4}=E\right), \overrightarrow{\mathbf{p}}=\left\{p_{i}\right\}$, (for $a=1,2,3,4)$, at the Planck scale $\ell_{p}:=\sqrt{\hbar G / c^{3}} \sim$ $10^{-33} \mathrm{~cm}$. In this work, the light velocity is fixed $c=1$ for a respective system of physical units. The type of modification $\varpi$ is considered differently in various approaches to QG and (non)commutative MGTs, supergravity and (super) string models etc. Modifications of quantum mechanics and certain QG theories have been studied also for models when $\ell_{p}$ is replaced by $\ell s$ (a string length which is found in the analysis of high-energy string scattering), for soliton-like structures known as Dirichlet p-brane and "Dparticles" (Dirichlet 0-branes) could probe the structure of spacetime down to scales higher than $\ell_{s}$. Here we note that locally anisotropic MGTs with MDRs are studied also as candidates for explaining acceleration cosmology and dark energy, DE, and dark matter, DM, physics, see [15,79,130$133,175,186,187,194,202]$ and references therein.

Example 2.1 (MDRs in QG and cosmology) In QG and various cosmological scenarios for which the Hamiltonian equations of motion $\dot{x}^{i}=\partial H / \partial p^{i}$ (we can consider similarly some Lagrange equations with possible nonholonomic constraints) are still valid at least approximately, quantum effects are modeled by a deformed dispersion relation for photons, $c^{2} \mathbf{p}^{2}=E^{2}\left[1+f\left(E /{ }^{q g} E\right)\right]$, where ${ }^{q g} E$ is an effective quantum gravity scale and the function $f$ is model-dependent. For $E \ll q^{q g} E$, one expects a series extension of MDRs, $\left.c^{2} \mathbf{p}^{2}=E^{2}\left[1+\xi E /{ }^{q g} E+O\left[E /{ }^{q g} E\right]^{2}\right)\right]$, where $\xi= \pm 1$ should be fixed in a dynamical framework for a corresponding theory. Such extensions correspond to energy-dependent velocities $v=\frac{\partial E}{\partial p} \sim c\left(1-\xi E /{ }^{q g} E\right)$. These formulas are analogous to those for a conventional relativistic medium, such as a gravy-electromagnetic plasma, modeling a gravitational aether which is believed to contain microscopic quantum fluctuations which may occur for Planck values $\ell_{p}, t_{p} \sim 1 / E_{p}$, where $E_{p} \sim 10^{19} \mathrm{GeV}$.

The vacuum in above example is viewed as a non-trivial medium containing "foamy" quantum-gravity fluctuations which may include pair creations of virtual black holes, wormholes etc. One considers that $\xi=1$ and $c^{2} p^{2}=$ $\left({ }^{q g} E\right)^{2}\left[1-e^{E /{ }^{g g} E}\right]^{2}$ for theories with $\kappa$-deformation of Poincaré symmetries. MDRs can be also found in models with quantization of point particles in a discrete spacetime [51]. The modifications emerging at the level of the $\kappa$-Poincaré (Hopf) algebra [89], and other types noncommu- 
tative black holes [168,183], require physical interpretations on generalized Lorentz manifolds.

Our approach to geometrizing classical and quantum theories on (co)tangent Lorentz bundles (in this work, there are considered 3-d and, in the bulk, 4-d pseudo-Riemannian base manifolds) will be elaborated following three assumptions:

Assumption 2.1 (Background quadratic elements on total spaces of (co)tangent bundles) The are standard gravity and particle physics theories based on the special relativity and Einstein gravity principles and axioms. In such theories, the spacetime geometry is described by a four dimensional, 4-d, Lorentz spacetime manifold $V$ and respective (co)tangent bundles, $T V$ and/or $T^{*} V$, enabled with corresponding quadratic elements determined by total phase space metrics with signature $(+++-;+++-)$,

$$
\begin{aligned}
& d s^{2}=g_{\alpha \beta}\left(x^{k}\right) d u^{\alpha} d u^{\beta}=g_{i j}\left(x^{k}\right) d x^{i} d x^{j}+\eta_{a b} d y^{a} d y^{b}, \\
& \quad \text { for } y^{a} \sim d x^{a} / d \tau ; \quad \text { and/or } \\
& d^{\prime} s^{2}={ }^{\prime} g_{\alpha \beta}\left(x^{k}\right) d^{\prime} u^{\alpha} d^{\prime} u^{\beta} \\
& =g_{i j}\left(x^{k}\right) d x^{i} d x^{j}+\eta^{a b} d p_{a} d p_{b}, \quad \text { for } p_{a} \sim d x_{a} / d \tau .
\end{aligned}
$$

In formulas (2) and (3), the local frame and dual frame (coframe) coordinates are labeled in the forms: $u^{\alpha}=\left(x^{i}, y^{a}\right)$, (or in brief, $u=(x, y)$ ), on the tangent bundle $T V$; and ' $u^{\alpha}=\left(x^{i}, p_{a}\right)$, (or in brief, ' $u=(x, p)$ ), on the cotangent bundle $T^{*} V$. The pseudo-Riemannian spacetime metric $g=\left\{g_{i j}(x)\right\}$ can be a solution of the Einstein equations for the Levi-Civita connection $\nabla$. In diagonal form, the vertical metric $\eta_{a b}$ and its dual $\eta^{a b}$ are standard Minkowski metrics, $\eta_{a b}=\operatorname{diag}[1,1,1,-1]$ used for computations in typical fibers for certain bundle geometric and physical models. The geometric and physical models are elaborated for general frame/coordinate transforms in total spaces when the metric structures can be parameterized equivalently by the same hcomponents of $g_{\alpha \beta}\left(x^{k}\right)$ and ' $g_{\alpha \beta}\left(x^{k}\right)=g_{\alpha \beta}\left(x^{k}\right)$ in quadratic elements (2) and (3). Curves $x^{a}(\tau)$ on $V$ are parameterized by a positive parameter $\tau .^{3}$

Assumption 2.2 (Nonlinear quadratic elements for modeling Finsler-Lagrange-Hamilton geometries on (co)tangent bundles) MGTs and quasi-classical limits of QG are characterized by MDRs (1) with possible small values of indicator $\varpi$ are described by basic Lorentzian and nonRiemannian total phase space geometries determined by nonlinear quadratic line elements

\footnotetext{
${ }^{3}$ In this work, there are such conventions for indices: the "horizontal" indices, h-indices, run values $i, j, k, \ldots=1,2,3,4$; the vertical indices, v-vertical, run values $a, b, c \ldots=5,6,7,8$; respectively, the v-indices can be identified/contracted with h-indices 1, 2, 3, 4 for lifts on total (co)tangent Lorentz bundles, when $\alpha=(i, a), \beta=(j, b), \gamma=$ $(k, c), \ldots=1,2,3, \ldots 8$. We shall use letters labelled by an abstract left up/low symbol "।" (for instance, ' $u^{\alpha}$ and ' $g_{\alpha \beta}$ ) in order to emphasize that certain geometric/physical objects are defined on $T^{*} V$.
}

$d s_{L}^{2}=L(x, y), \quad$ for models on $T V ;$

$d^{\prime} s_{H}^{2}=H(x, p)$, for models on $T^{*} V$.

This assumption involves geometric/physical theories with an effective phase spacetime modelled by generalized frame, metric and linear and nonlinear connection structures defined on (co)tangent Lorentz bundles. For localized zero indicators in (1), $\varpi=0$, the nonlinear quadratic line elements (4) and (5) transform correspondingly into linear quadratic elements (2) and (3).

In modern literature on geometric mechanics, kinetics and statistical mechanics of locally anisotropic processes, classical MGTs, and QG, (see [147-150,155, 195,197,200]), there were studied a series of important examples involving such concepts:

Example 2.2 (Relativistic models of Hamilton geometry and phase spaces) A 4-d relativistic model of Hamilton space $H^{3,1}=\left(T^{*} V, H(x, p)\right)$ is determined by a fundamental function (equivalently, generating Hamilton function) on a Lorentz manifold $V$, constructed as $T^{*} V \ni(x, p) \rightarrow$ $H(x, p) \in \mathbb{R}$, which defines a real valued function being differentiable on $\widehat{T^{*} V}:=T^{*} V /\left\{0^{*}\right\}$, for $\left\{0^{*}\right\}$ being the null section of $T^{*} V$, and continuous on the null section of $\pi^{*}: T^{*} V \rightarrow V$. Such a model is regular if the Hessian (cv-metric)

$$
{ }^{\prime} \widetilde{g}^{a b}(x, p):=\frac{1}{2} \frac{\partial^{2} H}{\partial p_{a} \partial p_{b}}
$$

is non-degenerate, i.e. det $\left|{ }^{\prime} \widetilde{g}^{a b}\right| \neq 0$, and of constant signature.

For elaborating physical and mechanical models, one follows different geometric and physical principles in order to define a (non)relativistic Hamiltonian, i.e generating function, $H(x, p)$. Such a function may describe propagation of test particles, or perturbations of scalar fields in an effective phase space, various noncommutative generalizations, quantum fluctuations etc.

Remark 2.1 (From MDR-indicators to Hamilton spaces) For any MDR of type (1), we can construct a Hamilton space $H^{3,1}$ if the effective Hamilton function is defined

$$
H(p):=E= \pm\left(c^{2} \overrightarrow{\mathbf{p}}^{2}+c^{4} m^{2}-\varpi\left(E, \overrightarrow{\mathbf{p}}, m ; \ell_{P}\right)\right)^{1 / 2}
$$

This describes a Hamilton like geometry of relativistic point particles propagating in a cv-space with MDRs. In general, such a propagation is in an effective phase space endowed with local coordinates $\left(x^{i}, p_{a}\right)$ and generalized indicator $\varpi\left(x^{i}, E, \overrightarrow{\mathbf{p}}, m ; \ell_{P}\right)(1)$.

Changing the system of frames/coordinates on total space, we obtain generating functions of type $H(x, p)$. We can use for geometric modeling certain general (for simplicity, regular) generating functions $H(x, p)$ on $T^{*} V$. 
Example 2.3 (Relativistic Lagrange spaces) A relativistic 4d model of Lagrange space $L^{3,1}=(T V, L(x, y))$ is defined by a fundamental function (equivalently, generating function) $T V \ni(x, y) \rightarrow L(x, y) \in \mathbb{R}$, i.e. a real valued function which is differentiable on $\widetilde{T V}:=T V /\{0\}$, for $\{0\}$ being the null section of $T V$, and continuous on the null section of $\pi: T V \rightarrow V$. Such a model is regular if the Hessian (v-metric)

$\widetilde{g}_{a b}(x, y):=\frac{1}{2} \frac{\partial^{2} L}{\partial y^{a} \partial y^{b}}$

is non-degenerate, i.e. $\operatorname{det}\left|\widetilde{g}_{a b}\right| \neq 0$, and of constant signature.

The values $\widetilde{g}_{a b}$ and ' $\widetilde{g}^{a b}$ are labeled by tilde " $\sim$ " in order to emphasize that such conventional $\mathrm{v}-$ metrics are defined canonically by respective Lagrange and Hamilton generating functions, which may encode various types of MDRs and LVs terms. Considering general frame/coordinate transforms on $T V$ and/or $T^{*} V$, we can express any "tilde" Hessian in a general quadratic form, respectively as a vertical metric (v-metric), $g_{a b}(x, y)$, and/or co-vertical metric (cv-metric), ${ }^{\prime} g^{a b}(x, p)$. Inversely, prescribing any v-metric (cv-metric), we can introduce respective (co)frame/coordinate systems, when such values can transformed into certain canonical ones, with "tilde" values. In general, a $g_{a b}$ is different from the inverse of ' $g^{a b}$, i.e. from ' $g_{a b}$. We shall omit tildes on geometrical/physical objects if certain formulas hold in general (not only canonical) forms and/or that will not result in ambiguities.

Remark 2.2 (Legendre transforms and $\mathcal{L}$-duality of Lagrange and Hamilton spaces) There are Legendre transforms $L \rightarrow$ $H$, with $H(x, p):=p_{a} y^{a}-L(x, y)$ and $y^{a}$ determining solutions of the equations $p_{a}=\partial L(x, y) / \partial y^{a}$. In a similar manner, the inverse Legendre transforms can be introduced, $H \rightarrow L$, for

$L(x, y):=p_{a} y^{a}-H(x, p)$

and $p_{a}$ determining solutions of the equations $y^{a}=$ $\partial H(x, p) / \partial p_{a}$.

For further details on relativistic Lagrange and Hamilton spaces, with reviews of non-relativistic original results, readers may consult $[8,161,172,184]$, where theories with degenerate Hessians are also considered (we omit such constructions in this work). Here we cite a very important paper [75] where an original geometrization of Lagrange mechanics was proposed. The main idea was to drop the homogeneity condition for Finsler generating functions and apply in studies of properties of mechanical systems certain methods of Finsler and almost Kä hler geometry [91,92]. The geometry of Hamilton spaces consists a natural dualization on $T^{*} V$ of the Lagrange geometry on $T V$. Nevertheless, such constructions are not completely dual because of Legendre transforms and "symplectomorphisms", see details in the mentioned references. In general, the Hamilton mechanics is not equivalent to the Lagrange mechanics and different almost symplectic realizations of such theories can be elaborated. Using Lagrange or Hamilton geometries, we can model different types of MGTs on (co)tangent bundles. Only for certain well-defined conditions such theories and respective classes of solutions of motion/evolution equations are equivalent.

Example 2.4 (Finsler-Cartan geometries as particular cases of Lagrange-Hamilton spaces) A relativistic 4-d model of Finsler space is an example of Lagrange space when a regular $L=F^{2}$ is defined by a fundamental (generating) Finsler function subjected to the conditions: (1) $F$ is a real positive valued function which is differential on $\widetilde{T V}$ and continuous on the null section of the projection $\pi: T V \rightarrow V$; (2) it is satisfied the homogeneity condition $F(x, \lambda y)=|\lambda| F(x, y)$, for a nonzero real value $\lambda$; and (3) the Hessian (8) is defined by $F^{2}$ in such a form that in any point $\left(x_{(0)}, y_{(0)}\right)$ the v-metric is of signature $(+++-)$. In a similar form, there are defined relativistic 4-d Cartan spaces $C^{3,1}=(V, C(x, p))$, when $H=C^{2}(x, p)$ is 1-homogeneous on co-fiber coordinates $p_{a}$.

For simplicity, the bulk of geometric constructions in this work will be performed for (effective and/or generalized) Lagrange and Hamilton spaces considering that via corresponding frame and Legendre transforms, or homogeneity conditions, we can generate necessary type Lagrange/Finsler/ Cartan configurations. Nevertheless, a series of important formulas and proofs will be presented both on tangent and cotangent bundles in order to emphasize the generality of our geometric methods which can be applied to a large class of MGRs with different types of geometrization of classical and quantum physical theories. Another argument to dub the formulas will be that in many cases the (non)linear connections and/or almost symplectic structures are constructed differently on tangent and cotangent bundles. This results in different geometric and physical models (the (non)linear symmetries and related conservation laws are also different) even being written in abstract geometric forms the formulas are very similar. For certain well-defined geometric/physical conditions, it is possible to establish certain equivalence and/or duality of constructions but this is an issue of additional assumptions and a more rigorous analysis for geometric structures and fundamental geometric/physical equations.

Definition 2.1 (Nonlinear connections and nonholonomic $h-v$ and/or $h-c v$ splitting) A nonlinear connection, $\mathrm{N}$ connection, structure for $T V$, or $T^{*} V$, is defined as a Whitney sum of conventional $h$ and $v$-distributions, or $h$ and $c v$ distributions,

$\mathbf{N}: T T V=h T V \oplus v T V$ or 


$$
{ }^{\mathrm{I}} \mathbf{N}: T T^{*} V=h T^{*} V \oplus v T^{*} V .
$$

There were formulated different equivalent definitions of $\mathrm{N}$-connections on (co)tangent bundles and fibred manifolds, studied in $[8,161,172,184] .{ }^{4}$ In local form (parameterizing the corresponding $\mathrm{N}$-connections by coefficients $\mathbf{N}=\left\{N_{i}^{a}\right\}$ and $\mathbf{N}=\left\{{ }^{\prime} N_{i a}\right\}$ with respect to coordinate (dual) bases), one prove by explicit constructions:

Lemma 2.1 ( $N$-adapted (co)frames) A N-connection (10) defines respective systems of $N$-adapted bases

$$
\begin{aligned}
\mathbf{e}_{\alpha} & =\left(\mathbf{e}_{i}=\frac{\partial}{\partial x^{i}}-N_{i}^{a}(x, y) \frac{\partial}{\partial y^{a}}, e_{b}=\frac{\partial}{\partial y^{b}}\right), \\
\mathbf{e}^{\alpha} & =\left(e^{i}=d x^{i}, \mathbf{e}^{a}=d y^{a}+N_{i}^{a}(x, y) d x^{i}\right),
\end{aligned}
$$

\footnotetext{
4 The concept of $\mathrm{N}$-connection is equivalent to that of Ehresmann connection in differential geometry [44]. In coordinate form, Nconnections are used in the first monograph on Finsler geometry [27]. The N-connection and Finsler geometries were studied originally in $[72,73]$. The formalism of $\mathrm{N}$-connections was introduced and developed in the Einstein/string/brane/gauge theories [42,149$151,158,166,168,171,175]$. On 4-d pseudo-Riemannian manifolds, a $\mathrm{N}$-connection can be defined as a conventional nonholonomic $2+2$ splitting by considering certain local ( $\mathrm{N}$-adapted) bases $\mathbf{e}_{\mu}=\left(\mathbf{e}_{i}, e_{a}\right)$ and their duals $\mathbf{e}^{v}=\left(e^{j}, \mathbf{e}^{b}\right)$,
}

$$
\begin{aligned}
\mathbf{e}_{i} & =\frac{\partial}{\partial x^{i}}-N_{i}^{c} \frac{\partial}{\partial y^{c}}, e_{a}=\partial_{a}=\frac{\partial}{\partial y^{a}} \text { and } \\
e^{j} & =d x^{j}, \mathbf{e}^{b}=d y^{b}+N_{k}^{b} d x^{k} .
\end{aligned}
$$

In this footnote, indices run values $i, j, k \ldots=1,2 ; a, b, c, \ldots=3,4$ and $\alpha, \beta, \ldots=1,2,3,4$ for $u^{4}=t$ being a time like coordinate. Such frames are called nonholonomic satisfying, in general, the relations $\left[\mathbf{e}_{\alpha}, \mathbf{e}_{\beta}\right]=\mathbf{e}_{\alpha} \mathbf{e}_{\beta}-\mathbf{e}_{\beta} \mathbf{e}_{\alpha}=W_{\alpha \beta}^{\gamma} \mathbf{e}_{\beta}$. If the anholonomy coefficients $W_{i a}^{b}=\partial_{a} N_{i}^{b}, W_{j i}^{b}=\Omega i j^{b}=\mathbf{e}_{j}\left(N_{i}^{b}\right)-\mathbf{e}_{i}\left(N_{j}^{b}\right)$ are zero, we get holonomic bases which allows to consider some coordinate transforms when $\mathbf{e}_{\alpha} \rightarrow \partial_{\alpha}$ and $\mathbf{e}^{\beta} \rightarrow d u^{\beta}$. On 8-d (co)tangent Lorentz bundles, the $\mathrm{N}$-connections and respective $\mathrm{N}$-adapted frames are defined in the forms (10) and (11) and (12). With respect to $\mathrm{N}$-adapted bases, one say that a vector, a tensor and other geometric objects are represented by $\mathrm{N}$-adapted coefficients and called correspondingly as a distinguished vector (d-vector), a distinguished tensor (d-tensor) and distinguished objects (d-object)

The geometry of $\mathrm{N}$-connections is related to the geometry of nonholonomic manifolds in the sense considered by G. Vrănceanu $[211,212]$, see a review of former results in [19]. In brief, a nonholonomic manifold is a usual one endowed with a nonholonomic distribution, for instance, defined by a nonholonomic frame structure. N-connection and nonholonomic geometric methods were used for elaborating the AFDM. Coordinate free and global approaches to Finsler geometry and various relativistic/string/brane/gauge generalizations were developed in $[153,161,169,219]$.

$$
\begin{aligned}
\text { and/or }{ }^{\prime} \mathbf{e}_{\alpha} & =\left({ }^{\prime} \mathbf{e}_{i}=\frac{\partial}{\partial x^{i}}-{ }^{\prime} N_{i a}(x, p) \frac{\partial}{\partial p_{a}},{ }^{\prime} e^{b}=\frac{\partial}{\partial p_{b}}\right), \\
{ }^{\prime} \mathbf{e}^{\alpha} & =\left({ }^{\prime} e^{i}=d x^{i}, \quad \mathbf{e}_{a}=d p_{a}+{ }^{\prime} N_{i a}(x, p) d x^{i}\right) .
\end{aligned}
$$

In our works, boldface symbols are used in order to emphasize that certain geometric/physical objects are considered in $\mathrm{N}$-adapted form for certain spaces enabled with $\mathrm{N}$-connection structure and when the coefficients of tensors, spinors, and fundamental geometric objects can be computed with respect to N-elongated bases of type (11) and/or (12). A splitting (10), and respective $\mathrm{N}$-adapted bases, defines corresponding non-integrable (equivalently, nonholonomic, or anholonomic) structures on (co)tangent Lorentz bundles and transforms such spaces into nonholonomic manifolds, see details in Refs. [19,171,175,186,211,212].

The sets of N-connection coefficients and necessary types of (co)frame/coordinate transforms can be used for constructing lifts of metric structures $(V, g)$ to respective nonholonomic (co)tangent bundles, ( $\mathbf{T V}, \mathbf{N}, \mathbf{g})$ and $\left(\mathbf{T}^{*} \mathbf{V},{ }^{\prime} \mathbf{N},{ }^{\prime} \mathbf{g}\right)$.

Assumption 2.3 (d-metrics on (co)tangent Lorentz bundles) The total spaces of tangent, $\mathbf{T V}$, and cotangent, $\mathbf{T}^{*} \mathbf{V}$, Lorentz bundles used for elaborating physical theories with MDRs and LVs generalizations of the Einstein gravity are enabled, respectively, with pseudo-Riemannian metric, $\mathbf{g}$, and ' $\mathbf{g}$, structures. Such metrics can be parameterized by frame transforms in $\mathrm{N}$-adapted form, i.e. as distinguished metrics (dmetrics)

$$
\begin{aligned}
\mathbf{g} & =\mathbf{g}_{\alpha \beta}(x, y) \mathbf{e}^{\alpha} \otimes \mathbf{e}^{\beta} \\
& =g_{i j}(x) e^{i} \otimes e^{j}+\mathbf{g}_{a b}(x, y) \mathbf{e}^{a} \otimes \mathbf{e}^{a} \text { and } / \text { or } \\
{ }^{\prime} \mathbf{g} & ={ }^{\prime} \mathbf{g}_{\alpha \beta}(x, p){ }^{\prime} \mathbf{e}^{\alpha} \otimes{ }^{\prime} \mathbf{e}^{\beta} \\
& =g_{i j}(x) e^{i} \otimes e^{j}+{ }^{\prime} \mathbf{g}^{a b}(x, p)^{\prime} \mathbf{e}_{a} \otimes{ }^{\prime} \mathbf{e}_{b} .
\end{aligned}
$$

In this paper, we shall work with metrics on 8-d manifolds of signature $(+,+,+,-,+,+,+,-))$. A pseudoRiemannian metric $g_{i j}(x)$ can be subjected to the condition that it defines a solution of the standard Einstein equations in GR and a corresponding Lorentz manifold V. Such constructions for the base spacetime manifold $V$ are possible for the Levi-Civita connection completely determined by $g_{i j}(x)$ by imposing the metric compatibility and zero torsion conditions. Working with more general classed of geometric/physical models with general MDRs and nonholonomic (co)frame structures elaborated on (co)tangent Lorentz bundles, we have to introduce into consideration non-Riemannian geometries with more general metric and connection structures. There are necessary additional geometrically and physically motivated assumptions on how nonlinear quadratic elements of type (4), or (5), and/or (13), or (14), encode MDRs. 
2.2 Canonical N-connections, metrics and almost symplectic structures

We shall follow a coordinate-free formalism for nonholonomic manifolds and bundles enabled with $\mathrm{N}$-connection structure. Certain important formulas and results will be formulated in coefficient forms, with respect to $\mathrm{N}$-adapted frames, which is important for constructing in explicit form exact and parametric solutions following the AFDM.

\subsubsection{MDR, Hamilton-Lagrange generating functions and $N$-connections}

Let us consider a spacetime Lorentzian manifold $\mathbf{V}$ modeled as a pseudo-Riemannian manifold endowed with a metric $h g=\left\{g_{i j}(x)\right\}$ of signature $(3,1)$. Such metrics can be deformed by off-diagonal/nonholonomic transforms to metrics depending on velocity/momentum coordinates.

Proposition 2.1 A MDR(1) defines naturally canonical data for a v-metric (Hessian) $\widetilde{g}_{a b}(x, p)$ (8) and a nonlinear quadratic element (5) determining a relativistic model of Hamilton space $\widetilde{H}^{3,1}=\left(T^{*} V, \widetilde{H}(x, p)\right)$.

Proof Let us fix a point $x_{[0]} \in \mathbf{V}$, with a fiber space of co-vectors in this point ${ }^{\prime} u\left[x_{[0]}\right]=\left(x_{[0]}, p\right) \in T_{x_{[0]}}^{*} V$, and consider an effective Hamilton function $H\left(x_{[0]}, p\right)(7)$ determined by a nontrivial MDR (1) with nontrivial indicator $\varpi$. The generating Hamilton function is defined as a union of all points $x \in U_{x_{[0]}} \subset \mathbf{V}, \widetilde{H}(x, p):=\bigcup_{x, U} H\left(x_{[0]}, p\right)$ for an atlas of unions of carts $U_{x}$ covering $\mathbf{V}$. Tilde " $\sim$ ", on symbols will be used in this work in order to emphasize that certain spaces and geometric/physical values are written in a "canonical" adapted form determined by certain MDR and associated Hamilton, $\widetilde{H}$, and/or Lagrange, $\widetilde{L}$, structures. In a constructive approach, one prescribes a necessary smooth class (for instance, analytic or of some finite class of differentiability) fundamental function $H(x, p)$ on $T^{*} V$ determining for any fixed $x=x_{[0]}$ a MDR. Such a relativistic Hamilton space is regular a point ${ }^{\prime} u=(x, p)$ if $\widetilde{g}_{a b}$ is not degenerate in this point. For $\varpi=0$ (1), the cotangent bundle admits frame/coordinate transforms when the nonlinear quadratic element (5) can be parameterized as a linear quadratic element (3). Considering arbitrary frame/coordinate transform on $\mathbf{V}$ and/or $T^{*} V$, the geometric objects transforms into general ones which can be parameterized by symbols without tilde.

Consequence 2.0 (Canonical Lagrange-Hamilton spaces determined by MDRs) A MDR (1) defines $L$-dual, i.e. related via Legendre transforms, canonical relativistic models of Hamilton space $\widetilde{H}^{3,1}=\left(T^{*} V, \widetilde{H}(x, p)\right)$ and Lagrange space $\widetilde{L}^{3,1}=(T V, \widetilde{L}(x, y))$.
Proof It follows from a inverse Legendre transform (9), when $\widetilde{L}(x, y):=p_{a} y^{a}-\widetilde{H}(x, p)$ and $p_{a}$ taken as a solution of the equations $y^{a}=\partial \widetilde{H}(x, p) / \partial p_{a}$.

Let us consider a regular curve $c(\tau)$ defined $c: \tau \in$ $[0,1] \rightarrow x^{i}(\tau) \subset U \subset V$, for a real parameter $\tau$. Such a curve can be lifted to $\pi^{-1}(U) \subset \widetilde{T V}$ defining a curve in the total space, when $\widetilde{c}(\tau): \tau \in[0,1] \rightarrow$ $\left(x^{i}(\tau), y^{i}(\tau)=d x^{i} / d \tau\right)$ with a nonvanishing v-vector field $d x^{i} / d \tau$.

There are on $T^{*} V$ a canonical symplectic structure $\theta:=$ $d p_{i} \wedge d x^{i}$ and a unique vector filed

$\tilde{X}_{H}:=\frac{\partial \widetilde{H}}{\partial p_{i}} \frac{\partial}{\partial x^{i}}-\frac{\partial \widetilde{H}}{\partial x^{i}} \frac{\partial}{\partial p_{i}}$

defined by $\widetilde{H}$, following the equation $i \widetilde{X}_{H} \theta=-d \tilde{H}$. In above formulas $\wedge$ is the antisymmetric product and $i_{\widetilde{X}_{H}}$ denotes the interior produce defined by $\widetilde{X}_{H}$. In result, we can formulate and prove using an explicit calculus for any functions ${ }^{1} f(x, p)$ and ${ }^{2} f(x, p)$ on $T^{*} V$ :

Conclusion 2.1 A MDR (1) determines a canonical Poisson structure $\left\{{ }^{1} f,{ }^{2} f\right\}:=\theta\left(\widetilde{X}_{1 f}, \widetilde{X}_{2 f}\right)$.

Motion of probing point particles in a phase space modeled by an effective $\widetilde{H}^{3,1}$ are described by an effective relativistic Hamilton mechanics induced by MDRs as follows from

Corollary 2.1 For any effective Hamilton phase space model on $T^{*} V$, one holds the canonical Hamilton-Jacobi equations $\frac{d x^{i}}{d \tau}=\left\{\tilde{H}, x^{i}\right\}$ and $\frac{d p_{a}}{d \tau}=\left\{\tilde{H}, p_{a}\right\}$.

Proof It follows from the previous Conclusion.

Following a standard variational calculus (see similar details, for instance, in Ref. [8]), one obtains the proof of

Theorem 2.1 (Semi-sprays induced by MDRs and canonical Hamilton-Jacobi and Euler-Lagrange equations) The dynamics of a probing point particle in $L$-dual effective phase spaces $\widetilde{H}^{3,1}$ and $\widetilde{L}^{3,1}$ is described by fundamental generating functions $\widetilde{H}$ and $\widetilde{L}$ determined canonically by MDRs (1) and satisfy the Hamilton-Jacobi equations written equivalently as

$\frac{d x^{i}}{d \tau}=\frac{\partial \widetilde{H}}{\partial p_{i}}$ and $\frac{d p_{i}}{d \tau}=-\frac{\partial \widetilde{H}}{\partial x^{i}}$,

or as Euler-Lagrange equations,

$\frac{d}{d \tau} \frac{\partial \widetilde{L}}{\partial y^{i}}-\frac{\partial \widetilde{L}}{\partial x^{i}}=0$

which, in their turn, are equivalent to the nonlinear geodesic (semi-spray) equations

$\frac{d^{2} x^{i}}{d \tau^{2}}+2 \widetilde{G}^{i}(x, y)=0$, 
for $\widetilde{G}^{i}=\frac{1}{2} \widetilde{g}^{i j}\left(\frac{\partial^{2} \widetilde{L}}{\partial y^{i}} y^{k}-\frac{\partial \widetilde{L}}{\partial x^{i}}\right)$, with $\widetilde{g}^{i j}$ being inverse to $\widetilde{g}_{i j}$ (8).

It should be noted that the equations (15) emphasize that point like probing particles move not along usual geodesics as on Lorentz manifolds but follow some nonlinear geodesic equations determined by MDRs and/or LIVs.

Using above Theorem and by construction on open sets covering $V, T V$ and $T^{*} V$ (see Definition 2.1), one proves

Theorem 2.2 (Existence of canonical N-connections determined by MDRs and pseudo Hamilton and/or Lagrange generating functions) There are canonical $N$-connections determined by MDRs in L-dual form following formulas

$$
\begin{aligned}
{ }^{\prime} \widetilde{\mathbf{N}}= & \left\{{ }^{\prime} \widetilde{N}_{i j}:=\frac{1}{2}\left[\left\{{ }^{\prime} \widetilde{g}_{i j}, \widetilde{H}\right\}-\frac{\partial^{2} \widetilde{H}}{\partial p_{k} \partial x^{i}}{ }^{\prime} \widetilde{g}_{j k}\right.\right. \\
& \left.\left.-\frac{\partial^{2} \tilde{H}}{\partial p_{k} \partial x^{j}}{ }^{\prime} \widetilde{g}_{i k}\right]\right\} \text { and } \tilde{\mathbf{N}}=\left\{\widetilde{N}_{i}^{a}:=\frac{\partial \widetilde{G}}{\partial y^{i}}\right\},
\end{aligned}
$$

where ${ }^{\prime} \widetilde{g}_{i j}$ is inverse to ${ }^{\prime} \widetilde{g}^{a b}(6)$.

Hereafter we shall consider that using necessary type frame/coordiante transforms we can always establish (if necessary) certain nonholonomic frames and geometric variables determined by MDRs and respective $L$-dual relations between geometric/physical values on tangent and cotangent Lorentz bundles.

Introducing the canonical N-connection coefficients defined by this Theorem, respectively, into formulas (11) and (12) introduced by Lemma 2.1, we prove

Proposition 2.2 (Canonical N-adapted frames determined by MDRs and/or Lagrange-Hamilton generating functions) The $N$-connection structures $\widetilde{\mathbf{N}}$ and $\widetilde{\mathbf{N}}$ define respective systems of $N$-adapted (co)frames

$$
\begin{aligned}
\widetilde{\mathbf{e}}_{\alpha} & =\left(\widetilde{\mathbf{e}}_{i}=\frac{\partial}{\partial x^{i}}-\widetilde{N}_{i}^{a}(x, y) \frac{\partial}{\partial y^{a}}, e_{b}=\frac{\partial}{\partial y^{b}}\right), \text { on } T V ; \\
\widetilde{\mathbf{e}}^{\alpha} & =\left(\widetilde{e}^{i}=d x^{i}, \widetilde{\mathbf{e}}^{a}=d y^{a}+\widetilde{N}_{i}^{a}(x, y) d x^{i}\right), \text { on }(T V)^{*} ; \quad \text { (16) } \\
\text { and }{ }^{\prime} \widetilde{\mathbf{e}}_{\alpha} & =\left({ }^{\prime} \widetilde{\mathbf{e}}_{i}=\frac{\partial}{\partial x^{i}}-{ }^{\prime} \widetilde{N}_{i a}(x, p) \frac{\partial}{\partial p_{a}},{ }^{\prime} e^{b}=\frac{\partial}{\partial p_{b}}\right), \text { on } T^{*} V ; \\
\widetilde{\mathbf{e}}^{\alpha} & =\left({ }^{\prime} e^{i}=d x^{i},{ }^{\prime} \mathbf{e}_{a}=d p_{a}+{ }^{\prime} \widetilde{N}_{i a}(x, p) d x^{i}\right) \text { on }\left(T^{*} V\right)^{*} .
\end{aligned}
$$

We note that we can introduce canonical N-splitting

$$
\begin{aligned}
& \widetilde{\mathbf{N}}: T T V=h T V \oplus v T V \text { and/or } \\
& { }^{\prime} \widetilde{\mathbf{N}}: T T^{*} V=h T^{*} V \oplus v T^{*} V,
\end{aligned}
$$

respectively, on any tangent Lorentz bundle and cotangent Lorentz bundle by prescribing a system of so-called nonholonomic variables with $\mathrm{N}$-adapted frames of type (16) and (17). In such cases, probing point like particles are described by some effective models of Lagrange and/or Hamilton mechanics for the total phase spaces. If arbitrary frame and coordinate transforms are considered on such spacetime manifolds and phase (co)tangent bundle, the values with tilde transform correspondingly into arbitrary ones, i.e. into (10), (11) and (12).

Conclusion 2.2 (Canonical geometric data for LagrangeHamilton spaces) The nonholonomic structure of a Lorentz manifolds and respective (co)tangent bundles can be described in equivalent forms using canonical data $\left(\widetilde{L}, \widetilde{\mathbf{N}} ; \widetilde{\mathbf{e}}_{\alpha}, \widetilde{\mathbf{e}}^{\alpha}\right)$, with effective Lagrange density $\widetilde{L}$ (correspondingly, $\left(\widetilde{H},{ }^{\prime} \widetilde{\mathbf{N}}\right.$; $\widetilde{\mathbf{e}}_{\alpha}$, ' $\left.\widetilde{\mathbf{e}}^{\alpha}\right)$, with effective Hamilton density $\widetilde{H}$ ) or by a general $N$-splitting without effective Lagrangians (Hamiltonians), i.e. in terms of geometric data $\left(\mathbf{N} ; \mathbf{e}_{\alpha}, \mathbf{e}^{\alpha}\right)$ (correspondingly ( ' $\left.\mathbf{N} ; \quad \mathbf{e}_{\alpha},{ }^{\prime} \mathbf{e}^{\alpha}\right)$ ). Such structures are determined by a MDR (1) if, for instance, $\widetilde{H}$ is chosen following the procedure stated by Proposition 2.1. Such an analogous Lagrange (Hamilton) like interpretation of the geometry of phase spaces is "hidden" into the structure of nonholonomic manifolds/bundles endowed with $N$-connection splitting if general frame/coordinate transforms are considered on respective relativistic spacetimes and phase spaces.

The geometric constructions can be performed in equivalent forms for various nonholonomic data. For instance, we can consider arbitrary frame/coordinate transforms on (co)bundle spaces and write formulas without "tilde", i.e. in general (non canonical) forms, emphasizing certain $\mathrm{N}$ splitting, or in coordinate forms. Nevertheless, some classes nonholonomic variables can be more convenient for decoupling physically important systems of nonlinear PDEs, generating new classes of exact/parametric solutions, and other classes of nonholonomic variables can be more convenient for elaborating and effective/analogous geometric mechanics interpretation, or (for instance) for deformation quantization of MGTs and the Einstein gravity, see Refs. [8, 174, 184], and next sections.

Vector fields on nonholonomic (co)tangent bundles are called d-vectors if they are written in a form adapted to a prescribed N-connection structure. For instance, we decompose

$$
\begin{aligned}
\mathbf{X} & =\widetilde{\mathbf{X}}^{\alpha} \widetilde{\mathbf{e}}_{\alpha}=\widetilde{\mathbf{X}}^{i} \widetilde{\mathbf{e}}_{i}+X^{b} e_{b} \\
& =\mathbf{X}^{\alpha} \mathbf{e}_{\alpha}=\mathbf{X}^{i} \mathbf{e}_{i}+X^{b} e_{b} \in T \mathbf{T V}, \\
{ }^{\prime} \mathbf{X} & ={ }^{\prime} \widetilde{\mathbf{X}}^{\alpha} \widetilde{\mathbf{e}}_{\alpha}={ }^{\prime} \widetilde{\mathbf{X}}^{i} \widetilde{\mathbf{e}}_{i}+{ }^{\prime} X_{b}{ }^{\prime} e^{b} \\
& ={ }^{\prime} \mathbf{X}^{\alpha}{ }^{\prime} \mathbf{e}_{\alpha}={ }^{\prime} \mathbf{X}^{i}{ }^{\prime} \mathbf{e}_{i}+{ }^{\prime} X_{b}{ }^{\prime} e^{b} \in T \mathbf{T}^{*} \mathbf{V},
\end{aligned}
$$

for decompositions with respect to canonical, or arbitrary, Nadapted bases. In brief, one considers such $\mathrm{h}-\mathrm{v}$ and/or $\mathrm{h}-\mathrm{cv}$ decompositions, $\mathbf{X}^{\alpha}=\widetilde{\mathbf{X}}^{\alpha}=\left(\widetilde{\mathbf{X}}^{i}, X^{b}\right)=\left(\mathbf{X}^{i}, X^{b}\right),{ }^{\prime} \mathbf{X}^{\alpha}=$ ${ }^{\prime} \widetilde{\mathbf{X}}^{\alpha}=\left({ }^{\prime} \widetilde{\mathbf{X}}^{i},{ }^{\prime} X_{b}\right)=\left({ }^{\prime} \mathbf{X}^{i},{ }^{\prime} X_{b}\right)$.

We can write $\mathbf{X}$ and ' $\mathbf{X}$ as 1 -forms and $\mathrm{N}$-adapted canonical, or arbitrary, coefficients,

$$
\begin{aligned}
\mathbf{X} & =\widetilde{\mathbf{X}}_{\alpha} \mathbf{e}^{\alpha}=X_{i} e^{i}+\widetilde{\mathbf{X}}^{a} \widetilde{\mathbf{e}}_{a}=\widetilde{\mathbf{X}}_{\alpha} \mathbf{e}^{\alpha} \\
& =X_{i} e^{i}+\mathbf{X}^{a} \mathbf{e}_{a} \in T^{*} \mathbf{T} \mathbf{V}
\end{aligned}
$$




$$
\begin{aligned}
& \mathbf{X}={ }^{\prime} \widetilde{\mathbf{X}}_{\alpha}{ }^{\prime} \mathbf{e}^{\alpha}={ }^{\prime} X_{i}{ }^{\prime} e^{i}+{ }^{\prime} \widetilde{\mathbf{X}}^{a}{ }^{\prime} \widetilde{\mathbf{e}}_{a}={ }^{\prime} \widetilde{\mathbf{X}}_{\alpha}{ }^{\prime} \mathbf{e}^{\alpha} \\
& ={ }^{\prime} X_{i}{ }^{\prime} e^{i}+{ }^{i} \mathbf{X}^{a}{ }^{\prime} \mathbf{e}_{a} \in T^{*} \mathbf{T}^{*} \mathbf{V},
\end{aligned}
$$

or, in brief, $\mathbf{X}_{\alpha}=\widetilde{\mathbf{X}}_{\alpha}=\left(X_{i}, \widetilde{\mathbf{X}}^{a}\right)=\left(X_{i}, \mathbf{X}^{a}\right),{ }^{\prime} \mathbf{X}_{\alpha}={ }^{\prime} \widetilde{\mathbf{X}}_{\alpha}=$ $\left({ }^{\prime} X_{i},{ }^{\prime} \tilde{\mathbf{X}}^{a}\right)=\left({ }^{\prime} X_{i},{ }^{\prime} \mathbf{X}^{a}\right)$.

Considering tensor products of $\mathrm{N}$-adapted (co)frames, we can parameterized in $\mathrm{N}$-adapted forms (canonical or general ones) arbitrary tensors fields, called as d-tensors.

\subsubsection{Locally anisotropic metrics on (co)tangent Lorentz bundles}

We can introduce canonical metric structures determined by MDRs and respective Lagrange and/or Hamilton effective functions using the so-called Sasaki lifts [218] from a base manifold to the total (co)tangent bundles. Using formulas (8) and (6), Theorem 2.2 (on existence of canonical $\mathrm{N}$-connections) and Proposition 2.2 (on canonical geometric data for Lagrange-Hamilton spaces) and by construction, we prove the

Theorem 2.3 (Existence of canonical d-metrics completely defined by MDR and generating Lagrange-Hamilton functions) There are canonical d-metric structures $\widetilde{\mathbf{g}}$ and $\quad \widetilde{\mathbf{g}}$ completely determined by a MDR (1), respectively for data $\left(\widetilde{L}, \quad \widetilde{\mathbf{N}} ; \widetilde{\mathbf{e}}_{\alpha}, \widetilde{\mathbf{e}}^{\alpha} ; \widetilde{g}_{j k}, \widetilde{g}^{j k}\right)$ and/or $\left(\widetilde{H}, ' \widetilde{\mathbf{N}} ; \quad \widetilde{\mathbf{e}}_{\alpha}, \widetilde{\mathbf{e}}^{\alpha} ; \quad\right.$ ' $\widetilde{g}^{a b}$, ' $\left.\widetilde{g}_{a b}\right)$, metrics (d-metrics)

$$
\begin{aligned}
\widetilde{\mathbf{g}} & =\widetilde{\mathbf{g}}_{\alpha \beta}(x, y) \widetilde{\mathbf{e}}^{\alpha} \otimes \widetilde{\mathbf{e}}^{\beta} \\
& =\widetilde{g}_{i j}(x, y) e^{i} \otimes e^{j}+\widetilde{g}_{a b}(x, y) \widetilde{\mathbf{e}}^{a} \otimes \widetilde{\mathbf{e}}^{a} \text { and/or } \\
' \widetilde{\mathbf{g}} & ={ }^{\prime} \widetilde{\mathbf{g}}_{\alpha \beta}(x, p) \widetilde{\mathbf{e}}^{\alpha} \otimes \widetilde{\mathbf{e}}^{\beta} \\
& ={ }^{\prime} \widetilde{g}_{i j}(x, p) e^{i} \otimes e^{j}+{ }^{\prime} \widetilde{g}^{a b}(x, p){ }^{\prime} \widetilde{\mathbf{e}}_{a} \otimes \widetilde{\mathbf{e}}_{b} .
\end{aligned}
$$

One follows:

Corollary 2.2 By frame transforms, the canonical d-metric structures (18) and (19) [with tildes] can be written, respectively, in general d-metric forms (13) and (14) [without tildes].

This Corollary motivates the Assumption 2.3 on metric properties of (co)tangent Lorentz bundles endowed with MDRs.

There are also possible general vierbein transforms $e_{\alpha}=$ $e^{\underline{\alpha}}(u) \partial / \partial u^{\underline{\alpha}}$ and $e^{\beta}=e^{\beta}{ }_{\beta}(u) d u \underline{\beta}$, where the local coordinate indices are underlined in order to distinguish them from arbitrary abstract ones. In such formulas, the matrix $e^{\beta}{ }_{\beta}$ is inverse to $e^{\frac{\alpha}{\alpha}}$ for orthonormalized bases. For Hamilton like configurations, one writes ' $e_{\alpha}={ }^{\prime} e^{\underline{\alpha}}\left({ }^{\prime} u\right) \partial / \partial{ }^{\prime} u \underline{\alpha}$ and ' $e^{\beta}={ }^{\prime} e^{\beta}{ }_{\beta}\left({ }^{\prime} u\right) d{ }^{\prime} u^{\beta}$. It should be noted that there are not used boldface symbols for such transforms because an arbitrary decomposition (for instance, one can be considered as particular cases certain diadic $2+2+2+2$ splitting) is not adapted to a $\mathrm{N}$-connection structure. Introducing formulas of type (16) and (17), respectively, into (13) and (14) and regrouping with respect to local coordinate bases, one proves

Corollary 2.3 (Equivalent re-writing of d-metrics as offdiagonal metrics) With respect to local coordinate frames, any d-metric structures on $\mathbf{T V}$ and/or $\mathbf{T}^{*} \mathbf{V}$,

$$
\begin{aligned}
& \mathbf{g}=\mathbf{g}_{\alpha \beta}(x, y) \mathbf{e}^{\alpha} \otimes \mathbf{e}^{\beta}=g_{\underline{\alpha} \underline{\beta}}(x, y) d u^{\underline{\alpha}} \otimes d u^{\underline{\beta}} \text { and/or } \\
& ' \mathbf{g}={ }^{\prime} \mathbf{g}_{\alpha \beta}(x, p)^{\prime} \mathbf{e}^{\alpha} \otimes{ }^{\prime} \boldsymbol{e}^{\beta}={ }^{\prime} g_{\underline{\alpha} \underline{\beta}}(x, p) d^{\prime} u^{\underline{\alpha}} \otimes d^{\prime} u^{\prime} \underline{\beta},
\end{aligned}
$$

can be parameterized viaframe transforms, $\mathbf{g}_{\alpha \beta}=e^{\alpha} e^{\underline{\alpha}}{ }^{\underline{\beta}} g_{\underline{\alpha}} \underline{\beta}$ and ${ }^{\prime} \mathbf{g}_{\alpha \beta}={ }^{\prime} e^{\frac{\alpha}{\alpha}}{ }^{\prime} e^{\frac{\beta}{\beta}}{ }^{\prime} g_{\underline{\alpha}} \underline{\beta}$, in respective off-diagonal forms:

$$
\begin{aligned}
& g_{\underline{\alpha} \underline{\beta}}=\left[\begin{array}{cc}
g_{i j}(x)+g_{a b}(x, y) N_{i}^{a}(x, y) N_{j}^{b}(x, y) & g_{a e}(x, y) N_{j}^{e}(x, y) \\
g_{b e}(x, y) N_{i}^{e}(x, y) & g_{a b}(x, y)
\end{array}\right] \text { and/or }
\end{aligned}
$$

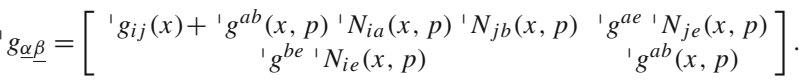

Parameterizations of type (20) are considered, for instance, in the Kaluza-Klein theory. Such metrics are generic offdiagonal if the corresponding $\mathrm{N}$-adapted structure is not integrable (see footnote 4). For MDR-generalizations of the Einstein gravity, we can consider that the h-metrics $g_{i j}(x)=$ ' $g_{i j}(x)$ are determined by a solution of standard Einstein equations but the terms with $N$-coefficients are determined by solutions of certain generalized gravitational field equations on nonholonomic phase spaces. In general, such solutions are not compactified on velocity/momentum like coordinates, $y^{a} / p_{a}$ like in standard Kaluza-Klein models. For decompositions with respect to coordinate bases of canonical d-metrics (18) and (19), we obtain coefficients of type (20) when (following a formal notation procedure) the geometric objects are labeled with tilde and $\tilde{g}_{i j}(x, y) \neq{ }^{\prime} \tilde{g}_{i j}(x, p)$. In result, we obtain

Conclusion 2.3 (Definition by MDRs of canonical frames and noncompactified Kaluza-Klein metrics for phase spacetimes with equivalent Lagrange-Hamilton interpretation) $A$ MDR-structure determines on (co)tangent Lorentz bundles nonholonomic generalized frame structures and equivalent $d$-metric and off-diagonal metric structures with dependencies on velocity/momentum type coordinates. For respective sets of nonholonomic variables, such locally anisotropic gravitational models admit analogous Lagrange and/or Hamilton mechanics interpretation, or as a generalized (nonholonomic) Kaluza-Klein theory without compactification on extra dimension (phase) coordinates.

Remark 2.3 (Two general classes of phase spaces and MGTs encoding MDTs and LIVs) There are two general classes of MGTs (on tangent and/or cotangent bundles) constructed with total metric structures determined by MDRs. In this 
work, we shall study with priority theories on cotangent Lorentz bundles $\mathbf{T}^{*} \mathbf{V}$ but also provide and compare important formulas for tangent Lorentz bundles (on TV and conventional fibered nonholonomic manifolds, such theories were studied in Refs. [31,35-38,68,201].

The type of MGT on a nonholonomic (co)tangent bundle, or nonholonomic manifold, depend on the type of metric and nonlinear and linear connection structures (see below) are involved for such constructions.

Remark 2.4 (Frame transforms, nonholonomic frame transforms and equivalence of canonical and noncanonical dmetric structures) If we fix a metric structure of type $\quad \widetilde{\mathbf{g}}(19)$, we can elaborate equivalent models with ' $\mathbf{g}$ (14) determined by certain classes of frame transforms. Inversely, prescribing a d-metric ' $\mathbf{g}$, we can define nonholonomic variables when this metric structure can be represented as a ' $\widetilde{\mathbf{g}}$. In such a model, ' $\mathbf{g}=\mathbf{} \mathbf{\mathbf { g }}$. Nevertheless, we can elaborate on bi-metric (and even multi-metric theories) if we consider that $\widetilde{\mathbf{g}}$ and ' $\mathbf{g}$ are related via certain generalized transforms considered, for instance, in bi-metric, bi-gravity, and/or massive gravity $[68,69,200-202]$.

Instead of (canonical) metric structures, we can define on nonholonomic (co)tangent bundles equivalent (canonical) almost symplectic structures (in both cases, the constructions are determined by a MDR (1)) as in next subsection.

\subsection{Almost Kähler Lagrange-Hamilton structures and MDRs}

Spacetime models encoding MDRs and LIVs and formulated as almost Kähler geometries for relativistic LagrangeHamilton configurations were studied in $[8,161]$. Further developments were elaborated for almost symplectic (algebroid, commutative and noncommutative) models of deformation/geometric quantization and/or geometric flow theories [140,172,174,177,184,197,204,206] and references therein. Fundamental ideas on almost Kähler realisation of Finsler and Lagrange geometry were proposed in [75,91,92]. In nonrelativistic form, K. Matsumoto and J. Kern results were applied in geometric mechanics by a series of Romanian authors whose works were summarized in monographs [101,102] (see footnote 2 on ethical and political issues related to publication of those books).

\subsubsection{Canonical almost complex structures and Neijenhuis fields}

Fundamental (generating) Lagrange-Hamilton functions and MDRs determine canonical models of almost Kähler geometry. Such nonholonomic variables can be introduced in classical and quantum MGTs on (co)tangent bundles. One holds:
Proposition 2.3 (Existence of canonical almost complex structures for Lagrange-Hamilton spaces) MDRs (1) determining canonical $N$-connections $\widetilde{\mathbf{N}}$ and ' $\widetilde{\mathbf{N}}$ following conditions of Theorem 2.2 define respectively canonical almost complex structures $\widetilde{\mathbf{J}}$, on $\mathbf{T V}$, and ' $\widetilde{\mathbf{J}}$, on $\mathbf{T}^{*} \mathbf{V}$.

Proof Let us introduce the linear operator $\widetilde{\mathbf{J}}$ acting on $\widetilde{\mathbf{e}}_{\alpha}=$ $\left(\widetilde{\mathbf{e}}_{i}, e_{b}\right)(16)$ a as follows: $\widetilde{\mathbf{J}}\left(\mathbf{e}_{i}\right)=-\widetilde{\mathbf{e}}_{n+i}$ and $\widetilde{\mathbf{J}}\left(e_{n+i}\right)=\widetilde{\mathbf{e}}_{i}$. This operator defines globally an almost complex structure $(\widetilde{\mathbf{J}} \circ \widetilde{\mathbf{J}}=-\mathbf{I}$ for $\mathbf{I}$ being the unity matrix) on $\mathbf{T V}$ completely determined for Lagrange spaces by a $\widetilde{L}(x, y)$.

On $\mathbf{T}^{*} \mathbf{V}$, we can consider a linear operator $\widetilde{\mathbf{J}}$ acting on ' $\mathbf{e}_{\alpha}=\left({ }^{\prime} \mathbf{e}_{i},{ }^{\prime} e^{b}\right)$ (17) following formulas $' \widetilde{\mathbf{J}}\left({ }^{\prime} \mathbf{e}_{i}\right)=$ $-{ }^{\prime} e^{n+i}$ and ${ }^{\prime} \tilde{\mathbf{J}}\left({ }^{\prime} e^{n+i}\right)={ }^{\prime} \mathbf{e}_{i}$. Then $\widetilde{\mathbf{J}}$ defines globally an almost complex structure ( $\tilde{\mathbf{J}} \circ \quad \widetilde{\mathbf{J}}=-\mathbf{I}$ for $\mathbf{I}$ being the unity matrix) on $\mathbf{T}^{*} \mathbf{V}$ completely determined for Hamilton spaces by a $\widetilde{H}(x, p)$.

We note that $\tilde{\mathbf{J}}$ and $\tilde{\mathbf{J}}$ are standard almost complex structures only for the Euclidean signatures, respectively, on $\mathbf{T V}$ and $\mathbf{T}^{*} \mathbf{V}$. Considering arbitrary frame/coordinate transforms, we can omit tildes and write $\mathbf{J}$ and ' $\mathbf{J}$. Inversely, we can state certain almost complex nonholonomic variables prescribing a MDR inducing respective canonical structures.

Definition 2.2 (Curvatures of canonical $N$-connections and almost complex structures) The canonical Neijenhuis tensor fields determined by MDRs for respective canonical almost complex structures $\widetilde{\mathbf{J}}$ on $\mathbf{T V}$ and/or ${ }^{~} \widetilde{\mathbf{J}}$ on $\mathbf{T}^{*} \mathbf{V}$, are considered as curvatures of respective $\mathrm{N}$-connections

$$
\begin{aligned}
& \widetilde{\mathbf{\Omega}}(\tilde{\mathbf{X}}, \tilde{\mathbf{Y}}):=-[\tilde{\mathbf{X}}, \tilde{\mathbf{Y}}]+[\tilde{\mathbf{J}} \widetilde{\mathbf{X}}, \widetilde{\mathbf{J}} \widetilde{\mathbf{Y}}]-\widetilde{\mathbf{J}}[\widetilde{\mathbf{J}} \tilde{\mathbf{X}}, \tilde{\mathbf{Y}}] \\
& -\widetilde{\mathbf{J}}[\widetilde{\mathbf{X}}, \widetilde{\mathbf{J}} \widetilde{\mathbf{Y}}] \text { and/or }
\end{aligned}
$$

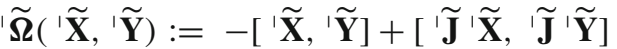

$$
\begin{aligned}
& \text { - ' } \widetilde{\mathbf{J}}\left[{ }^{\prime} \widetilde{\mathbf{J}} \cdot \widetilde{\mathbf{X}},{ }^{\prime} \tilde{\mathbf{Y}}\right]-{ }^{\prime} \tilde{\mathbf{J}}\left[{ }^{\prime} \tilde{\mathbf{X}},{ }^{\prime} \widetilde{\mathbf{J}}{ }^{\prime} \tilde{\mathbf{Y}}\right],
\end{aligned}
$$

for any d-vectors $\mathbf{X}, \mathbf{Y}$ and ' $\mathbf{X},{ }^{\prime} \mathbf{Y}$.

For arbitrary N-connection splitting, the formulas (21) can be written in general form without tilde values. Applying the left label "'”, we can rewrite geometric formulas on TV into respective ones on $\mathbf{T}^{*} \mathbf{V}$ (if necessary, for $\mathcal{L}$-dual values and with, or not "tildes"). One follows:

Corollary 2.4 In local coordinate form, a $\mathrm{N}$-connection on $\mathbf{T V}$, or $\mathbf{T}^{*} \mathbf{V}$, is characterized by such coefficients of Neijenhuis tensors (21), i.e. N-connection curvature,

$$
\begin{aligned}
\Omega_{i j}^{a}= & \frac{\partial N_{i}^{a}}{\partial x^{j}}-\frac{\partial N_{j}^{a}}{\partial x^{i}}+N_{i}^{b} \frac{\partial N_{j}^{a}}{\partial y^{b}}-N_{j}^{b} \frac{\partial N_{i}^{a}}{\partial y^{b}}, \text { or } \\
\Omega^{\prime} \Omega_{i j a}= & \frac{\partial^{\prime} N_{i a}}{\partial x^{j}}-\frac{\partial^{\prime} N_{j a}}{\partial x^{i}}+{ }^{\prime} N_{i b} \frac{\partial^{\prime} N_{j a}}{\partial p_{b}} \\
& -{ }^{\prime} N_{j b} \frac{\partial^{\prime} N_{i a}}{\partial p_{b}} .
\end{aligned}
$$


Some almost complex structures $\mathbf{J}$ and ' $\mathbf{J}$ transform into standard complex structures for Euclidean signatures if $\boldsymbol{\Omega}=$ 0 and/or $\mathbf{\Omega}=0$.

Remark 2.5 For almost complex structures determined by MDRs, formulas (22) can be written (using frame transforms) in respective canonical forms with "tilde" values determined by $\widetilde{\mathbf{N}}=\left\{\widetilde{N}_{j}^{b}\right\}$ and ' $\widetilde{\mathbf{N}}=\left\{{ }^{\prime} \widetilde{N}_{i a}\right\}$.

Using Proposition 2.2 (see also the footnote 4 on anholonomic frames and $\mathrm{N}$-connections), by straightforward $\mathrm{N}$ adapted calculus using formulas $\widetilde{\mathbf{e}}_{\alpha}=\left(\widetilde{\mathbf{e}}_{i}, e_{b}\right)(16), \quad \widetilde{\mathbf{e}}_{\alpha}=$ $\left(\widetilde{\mathbf{e}}_{i},{ }^{\prime} e^{b}\right)$ (17) and (22), we prove

Consequence 2.1 (Existence of MDR-induced canonic anholonomic frame structures) MDRs and respective canonical $\mathrm{N}$-connections induce canonical nonholonomic frame structures on $\mathbf{T V}$ and/or $\mathbf{T}^{*} \mathbf{V}$ characterized by corresponding anholonomy relations

$\left[\widetilde{\mathbf{e}}_{\alpha}, \widetilde{\mathbf{e}}_{\beta}\right]=\widetilde{\mathbf{e}}_{\alpha} \widetilde{\mathbf{e}}_{\beta}-\widetilde{\mathbf{e}}_{\beta} \widetilde{\mathbf{e}}_{\alpha}=\widetilde{W}_{\alpha \beta}^{\gamma} \widetilde{\mathbf{e}}_{\gamma}$

with (antisymmetric) anholonomy coefficients $\widetilde{W}_{i a}^{b}=\partial_{a} \widetilde{N}_{i}^{b}$ and $\widetilde{W}_{j i}^{a}=\widetilde{\Omega}_{i j}^{a}$, and

$\left[{ }^{\prime} \widetilde{\mathbf{e}}_{\alpha},{ }^{\prime} \widetilde{\mathbf{e}}_{\beta}\right]={ }^{\prime} \widetilde{\mathbf{e}}_{\alpha}{ }^{\prime} \widetilde{\mathbf{e}}_{\beta}-{ }^{\prime} \widetilde{\mathbf{e}}_{\beta}{ }^{\prime} \widetilde{\mathbf{e}}_{\alpha}={ }^{\prime} \widetilde{W}_{\alpha \beta}^{\gamma}{ }^{\prime} \widetilde{\mathbf{e}}_{\gamma}$

with anholonomy coefficients ' $\widetilde{W}_{i b}^{a}=\partial{ }^{\prime} \tilde{N}_{i b} / \partial p_{a}$ and ${ }^{\prime} \widetilde{W}_{j i a}={ }^{\prime} \widetilde{\Omega}_{i j a}$.

We note that we obtain holonomic (integrable) frame configurations if respective anholonomy coefficients (23) and/or (24) are zero.

Remark 2.6 (Generic off-diagonal metric structures canonically induced by MDRs) Canonical d-metric structures $\widetilde{\mathbf{g}}$ (18) and ' $\mathbf{g}(19)$ are described by generic off-diagonal metrics (20) if respective anholonomy coefficients (23) and (24) are not trivial. This means that MDRs (1) generate off-diagonal metric structures on $\mathbf{T V}$ and $\mathbf{T}^{*} \mathbf{V}$ if certain special conditions for integrability of respective frame structures and diagonalization (on some finite, or infinite phase space regions) are not imposed. It is necessary to elaborate more advanced and sophisticate geometric and numeric methods for constructing exact, parametric and approximate solutions with generic offdiagonal metrics and generalized connections (for instance, $\mathrm{BH}$, or cosmological type) in MGTs with MDRs.

\subsubsection{Canonical almost symplectic structures determined by MDRs}

The geometry of $\mathrm{N}$-connections and d-metric structures determined by MDRs and LIVs on nonholonomic (co)tangent Lorentz bundles can be described equivalently in terms of canonical almost symplectic variables. For relativistic generalizations and models of geometric flows and deformation and A-brane complexified quantization of EinsteinLagrange-Hamilton and generalized Finsler spaces, such constructions were elaborated in a series of our works $[8,12,161,172,174,177,184,197,204,206]$; on preliminary geometric ideas see $[45,75,91,92]$.

Definition 2.3 Almost symplectic structures on TV and $\mathbf{T}^{*} \mathbf{V}$ are defined by respective nondegenerate $\mathrm{N}$-adapted 2 forms

$\theta=\frac{1}{2} \theta_{\alpha \beta}(u) \mathbf{e}^{\alpha} \wedge \mathbf{e}^{\beta}$ and ${ }^{\prime} \theta=\frac{1}{2}{ }^{\prime} \theta_{\alpha \beta}(u){ }^{\prime} \mathbf{e}^{\alpha} \wedge \mathbf{e}^{\beta}$.

One holds the following

Proposition 2.4 For any $\boldsymbol{\theta}$ and ${ }^{\prime} \boldsymbol{\theta}$ on respective tangent and cotangent Lorentz bundle, there are unique $N$-connections $\mathbf{N}=\left\{N_{j}^{b}\right\}$ and $\mathbf{N}=\left\{{ }^{\prime} N_{i a}\right\}$ satisfying the conditions:

$$
\begin{aligned}
\theta & =(h \mathbf{X}, v \mathbf{Y})=0, \text { when } \theta=h \theta+v \theta, \quad \text { and } \\
{ }^{\prime} \theta & =\left(h^{\prime} \mathbf{X}, c v^{\prime} \mathbf{Y}\right)=0, \text { when }{ }^{\prime} \theta \doteq h^{\prime} \theta+c v^{\prime} \theta,
\end{aligned}
$$

for any $\mathbf{X}=h \mathbf{X}+v \mathbf{X}, \mathbf{Y}=h \mathbf{Y}+v \mathbf{Y}$ and $' \mathbf{X}=h^{\prime} \mathbf{X}+$ $c v^{\prime} \mathbf{X},{ }^{\prime} \mathbf{Y}=h^{\prime} \mathbf{Y}+c v^{\prime} \mathbf{Y}$, where

$$
h \theta(\mathbf{X}, \mathbf{Y}):=\theta(h \mathbf{X}, h \mathbf{Y}), v \theta(\mathbf{X}, \mathbf{Y}):=\theta(v \mathbf{X}, v \mathbf{Y}) ; \text { and }
$$

$h^{\prime} \theta\left({ }^{\prime} \mathbf{X},{ }^{\prime} \mathbf{Y}\right):={ }^{\prime} \theta\left(h^{\prime} \mathbf{X}, h^{\prime} \mathbf{Y}\right), c v^{\prime} \theta\left({ }^{\prime} \mathbf{X},{ }^{\prime} \mathbf{Y}\right)$

$$
:={ }^{\prime} \theta\left(c v^{\prime} \mathbf{X}, c v^{\prime} \mathbf{Y}\right) \text {. }
$$

Proof Let us sketch the proof on $\mathbf{T}^{*} \mathbf{V}$ for ${ }^{\prime} \theta$ (the constructions are similar on $\mathbf{T V}$ ).

For ${ }^{\prime} \mathbf{X}={ }^{\prime} \mathbf{e}_{\alpha}=\left({ }^{\prime} \mathbf{e}_{i},{ }^{\prime} e^{a}\right)$ and ${ }^{\prime} \mathbf{Y}={ }^{\prime} \mathbf{e}_{\beta}=\left({ }^{\prime} \mathbf{e}_{j},{ }^{\prime} e^{b}\right)$, as in (17), where $\quad \mathbf{e}_{\alpha}$ is a $\mathrm{N}$-adapted basis of type (12), we write the first equation in (25) in the form

$$
\begin{gathered}
{ }^{\prime} \theta={ }^{\prime} \theta\left({ }^{\prime} \mathbf{e}_{i},{ }^{\prime} e^{a}\right)={ }^{\prime} \theta\left(\frac{\partial}{\partial x^{i}}, \frac{\partial}{\partial p_{a}}\right) \\
-{ }^{\prime} N_{i b}{ }^{\prime} \theta\left(\frac{\partial}{\partial p_{b}}, \frac{\partial}{\partial p_{a}}\right)=0 .
\end{gathered}
$$

These conditions uniquely define ' $N_{i b}$ if ' $\theta$ is nondegenerate, i.e. $\left.\operatorname{rank}\right|^{\prime} \theta\left(\frac{\partial}{\partial p_{b}}, \frac{\partial}{\partial p_{a}}\right) \mid=4$. Setting locally

${ }^{\prime} \theta=\frac{1}{2}{ }^{\prime} \theta_{i j}(u) e^{i} \wedge e^{j}+\frac{1}{2}{ }^{\prime} \theta^{a b}(u)^{\prime} \mathbf{e}_{a} \wedge{ }^{\prime} \mathbf{e}_{b}$,

where the first term is for $h^{\prime} \theta$ and the second term is $c v^{\prime} \theta$, we get the second formula in (25).

In above Proposition, the constructed $\mathrm{N}$-connections are not canonical ones as in Theorem 2.2. There are necessary additional nonholonomic frame deformations/transforms in order to generate almost symplectic structures in canonical $\mathrm{N}$-adapted forms: A $\mathrm{N}$-connection ' $\mathbf{N}$ defines a unique decomposition of a d-vector ${ }^{\prime} \mathbf{X}=X^{h}+{ }^{\prime} X^{c v}$ on $T^{*} \mathbf{V}$, for $X^{h}=h^{\prime} \mathbf{X}$ and ${ }^{\prime} X^{c v}=c v{ }^{\prime} \mathbf{X}$, where the projectors $h$ and $c v$ defines respectively the dual distribution $\mathbf{N}$ on $\mathbf{V}$. They have the properties $h+c v=\mathbf{I}, h^{2}=h,(c v)^{2}=$ $c v, h \circ c v=c v \circ h=0$. This allows us to construct on $T^{*} \mathbf{V}$ 
the almost product operator ${ }^{\prime} \mathbf{P}:=I-2 c v=2 h-I$ acting on ${ }^{\prime} \mathbf{e}_{\alpha}=\left({ }^{\prime} \mathbf{e}_{i},{ }^{\prime} e^{b}\right)$ following formulas

$$
{ }^{\prime} \mathbf{P}\left({ }^{\prime} \mathbf{e}_{i}\right)={ }^{\prime} \mathbf{e}_{i} \text { and }{ }^{\prime} \mathbf{P}\left({ }^{\prime} e^{b}\right)=-{ }^{\prime} e^{b} .
$$

In a similar form, a $\mathrm{N}$-connection $\mathbf{N}$ induces an almost product structure $\mathbf{P}$ on $T \mathbf{V}$.

In geometric and physical models, there are used also the almost tangent (co)operators

$$
\begin{gathered}
\mathbb{J}\left(\mathbf{e}_{i}\right)=e_{4+i} \text { and } \mathbb{J}\left(e_{a}\right)=0, \quad \text { or } \mathbb{J}=\frac{\partial}{\partial y^{i}} \otimes d x^{i} ; \\
{ }^{\prime} \mathbb{J}\left(\mathbf{e}_{i}\right)={ }^{\prime} g_{i b}^{\prime} e^{b} \text { and }{ }^{\prime} \mathbb{J}\left({ }^{\prime} e^{b}\right)=0, \quad \text { or } \\
' \mathbb{J}={ }^{\prime} g_{i a} \frac{\partial}{\partial p_{a}} \otimes d x^{i} .
\end{gathered}
$$

The operators ' $\mathbf{P},{ }^{\prime} \mathbf{J}$ and ' $\mathbb{J}$ are respectively $\mathcal{L}$-dual to $\mathbf{P}, \mathbf{J}$ and $\mathbb{J}$ if and only if ' $\mathbf{N}$ and $\mathbf{N}$ are $\mathcal{L}$-dual and there are constructed respective (co)frame transforms to canonical values $\left[{ }^{\prime} \widetilde{\mathbf{P}},{ }^{\prime} \widetilde{\mathbf{J}},{ }^{\prime} \widetilde{\mathbb{J}}\right]$ and $[\widetilde{\mathbf{P}}, \widetilde{\mathbf{J}}, \widetilde{\mathbb{J}}]$.

For the above-introduced almost complex and almost product operators, we can check by straightforward computations the properties:

Proposition 2.5 Let $\left(\mathbf{N},{ }^{\prime} \mathbf{N}\right)$ be a pair of $\mathcal{L}$-dual $N$-connections. Then, we can construct canonical d-tensor fields (defined respectively by $L(x, y)$ and $H(x, p)$ related by Legendre transforms, see Remark on (9)):

$\mathbf{J}=-\delta_{i}^{a} e_{a} \otimes e^{i}+\delta_{a}^{i} \mathbf{e}_{i} \otimes \mathbf{e}^{a}$,

${ }^{\prime} \mathbf{J}=-{ }^{\prime} g_{i a}{ }^{\prime} e^{a} \otimes{ }^{\prime} e^{i}+{ }^{\prime} g^{i a}{ }^{\prime} \mathbf{e}_{i} \otimes{ }^{\prime} \mathbf{e}_{a}$

corresponding to the $\mathcal{L}$-dual pair of almost complex structures $\left(\mathbf{J},{ }^{\prime} \mathbf{J}\right)$,

$\mathbf{P}=\mathbf{e}_{i} \otimes e^{i}-e_{a} \otimes \mathbf{e}^{a}, \quad{ }^{\prime} \mathbf{P}={ }^{\prime} \mathbf{e}_{i} \otimes{ }^{\prime} e^{i}-{ }^{\prime} e^{a} \otimes{ }^{\prime} \mathbf{e}_{a}$

corresponding to the $\mathcal{L}$-dual pair of almost product structures $(\mathbf{P}, \quad$ 'P), and almost symplectic structures

$\theta=g_{a j}(x, y) \mathbf{e}^{a} \wedge e^{i}$ and ${ }^{\prime} \theta=\delta_{i}^{a}{ }^{\prime} \mathbf{e}_{a} \wedge{ }^{\prime} e^{i}$

The formulas in this Proposition can be re-written in canonical form by considering canonical $\mathrm{N}$-adapted bases with tilde. For instance, one re-writes (using frame transforms) (27) as $\tilde{\theta}=\widetilde{g}_{a j}(x, y) \widetilde{\mathbf{e}}^{a} \wedge e^{i}$ and ${ }^{\prime} \tilde{\theta}=\delta_{i}^{a}{ }^{\prime} \widetilde{\mathbf{e}}_{a} \wedge{ }^{\prime} e^{i}$.

For modeling of (co)tangent bundle N-connection and almost symplectic geometries on (co)tangent bundles with total dimension 8 , we introduce the

Definition 2.4 An almost Hermitian model of a tangent Lorentz bundle $T \mathbf{V}$ (or a cotangent Lorentz bundle $T^{*} \mathbf{V}$ ) equipped with a $\mathbf{N}$-connection structure $\mathbf{N}$ ( or ' $\mathbf{N}$ ) is defined by a triple $\mathbf{H}^{8}=(T \mathbf{V}, \theta, \mathbf{J})$, where $\theta(\mathbf{X}, \mathbf{Y}):=\mathbf{g}(\mathbf{J X}, \mathbf{Y})$ (or by a triple ${ }^{\prime} \mathbf{H}^{8}=\left(T^{*} \mathbf{V},{ }^{\prime} \theta,{ }^{\prime} \mathbf{J}\right)$, where ${ }^{\prime} \theta\left({ }^{\prime} \mathbf{X},{ }^{\prime} \mathbf{Y}\right):=$ ' $\mathbf{g}\left({ }^{\prime} \mathbf{J} ' \mathbf{X},{ }^{\prime} \mathbf{Y}\right)$ ). A space $\mathbf{H}^{8}$ (or ${ }^{\prime} \mathbf{H}^{8}$ ) is almost Kähler and denoted $\mathbf{K}^{8}$ if $d \theta=0$ (or ${ }^{\prime} \mathbf{K}^{8}$ if $d^{\prime} \theta=0$ ).
The following theorem holds:

Theorem 2.4 (MDR-induced canonical almost KählerLagrange/-Hamilton spaces) The Lagrange and Hamilton spaces (including those determined by MDRs (1)) can be represented respectively as canonical almost Kähler spaces (called almost Kähler-Lagrange and almost KählerHamilton) on TV and $\mathbf{T}^{*} \mathbf{V}$.

Proof It follows from the existence on $\mathbf{T V}$ and $\mathbf{T}^{*} \mathbf{V}$ of canonical N-connections under conditions of Theorem (2.2) and 1forms, respectively, defined by a regular Lagrangian $L$ and Hamiltonian $H$ (related by a Legendre transform), $\widetilde{\omega}=\frac{\partial L}{\partial y^{i}} e^{i}$ and ${ }^{\prime} \widetilde{\omega}=p_{i} d x^{i}$, for which $\tilde{\theta}=d \widetilde{\omega}$ and ${ }^{\prime} \tilde{\theta}=d{ }^{\prime} \tilde{\omega}$. As a result, we get that $d \theta=0$ and $d^{\prime} \tilde{\theta}=0$ corresponding to the Definition 2.4.

There are almost Kähler models on (co)tangent bundles defined canonically by (pseudo) Riemannian metrics on base manifolds with nonholonomic deformations to MGTs modeled as (effective phase) regular Lagrange and/or Hamilton spaces and respective almost Hermitian/Kähler spaces. In particular, we can consider almost Kähler-Finsler spaces originally elaborated in [91,92].

\subsection{Lagrange-Hamilton connections and curvatures}

The geometry of a (pseudo) Riemannian spacetime ( $V$, $\left.\left\{g_{i j}(x)\right\}\right)$ is completely determined by its metric structure $\left\{g_{i s}\right\}$. There is a uniquely defined by $\left\{g_{i s}\right\}$ linear connection called the Levi-Civita (LC) connection, $\nabla$, which by definition is torsionless and metric compatible. In GR, geodesic equations and auto parallel curves are described by equivalent systems on PDEs. For nontrivial MDRs and/or LIVs, point like probing particles and small perturbations of wave equations and scalar field equations do not move along usual geodesics (as in GR, on Lorentz manifolds) but follow certain curves described by nonlinear geodesic equations (15). In non-relativistic form, there were developed certain approaches related to Finsler geometry and semi-spray configurations [16,124,125], where the priority was given to the Chern connection for Finsler spaces. Such a connection is not compatible with the metric structure on the total bundle. This creates a number of ambiguities related to elaborating metric noncompatible Finsler gravity theories (including definition of spinors, definition of compatible motion equations and conservation laws), see explicit results, critics and discussions in Refs. [169,173,185,193].

The Theorem 2.2, Proposition 2.2 and Consequence 2.1 state explicitly that MDRs and LIVs define on (co)tangent Lorentz bundle certain generic off-diagonal metric structures (20) and related N-connection (10) and anholonomic frame structures characterized by respective Neijenhuis tensors 
(21). Elaborating on different types of Hamilton-LagrangeFinsler models encoding (1), we are not able to perform the constructions in $\mathrm{N}$-adapted anholonomic form if we work only with generalized (Finsler like) metrics determined by nonlinear quadratic forms $L(x, y)$ (4) and/or $H(x, p)(5)$. The geometry of nonholonomic (co)tangent Lorentz manifolds encodes new types of fundamental geometric/physical objects and more rich geometric structures. Physically viable nonholonomic deformations of GR on TV and $\mathbf{T}^{*} \mathbf{V}$ can be elaborated for locally anisotropic gravity and matter field theories if there are used certain well defined geometrically and physically motivated linear connection structures.

The goal of this subsection is to analyze which classes of linear connections and respective covariant derivative operators can be generated canonically by fundamental nonlinear quadratic forms and applied for formulating classical and quantum gravity and matter filed theories.

\subsubsection{Distinguished connections, $N$-adapted distortions and curvatures}

Let $D$ be a linear connection on TV when a $\mathcal{L}$-duality between the tangent and corresponding cotangent bundles can be defined by pull-back and push-forward maps (we omit geometric details on constructing such maps from/to base space to total space, considered, for instance, in Ref [8]). One defines a linear connection ${ }^{\prime} D$ on $\mathbf{T}^{*} \mathbf{V}$ as follows: ${ }^{\prime} D_{{ } \mathbf{X}}{ }^{\prime} \mathbf{Y}:=\left(D_{\mathbf{X}} \mathbf{Y}\right)^{*}={ }^{\prime}\left(D_{\mathbf{X}} \mathbf{Y}\right)$, for any vector fields ${ }^{\prime} \mathbf{X}$ and ${ }^{\prime} \mathbf{Y}$ on $\mathbf{T}^{*} \mathbf{V}$. Inversely, considering a linear connection

' $D$ on $\mathbf{T}^{*} \mathbf{V}$, we construct a linear connection ${ }^{\circ} D$ on $\mathbf{T V}$, following the rule ${ }^{\circ} D_{\mathbf{X}} \mathbf{Y}:=\left({ }^{\prime} D_{{ } \mathbf{X}}{ }^{\prime} \mathbf{Y}\right)^{\circ}$, for any vector fields $\mathbf{X}$ and $\mathbf{Y}$ on $\mathbf{T V}$.

On (co)tangent bundles, we can elaborate a geometry of affine (linear) connections and respective covariant derivatives in certain forms being (or not) adapted to a $\mathrm{N}$-connection structure.

Definition 2.5 (d-Connections as $N$-adapted linear connections) A distinguished connection (d-connection) is a linear connection $\mathbf{D}$ on $\mathbf{T V}$ ( or ' $\mathbf{D}$ on $\mathbf{T}^{*} \mathbf{V}$ ) which is compatible with the almost product structure $\mathbf{D P}=0$ ( or $\left.^{\prime} \mathbf{D}{ }^{\prime} \mathbf{P}=0\right)$. In equivalent form, such a d-connection is defined to preserve under parallelism a respective $\mathrm{N}$-connection splitting (10).

For a MDR (1), it is possible to construct $\mathcal{L}$-dual Lagrange and Hamilton spaces when $\mathbf{D P}=0$ induces ${ }^{\prime} \mathbf{D}{ }^{\prime} \mathbf{P}=0$, and inversely. In general, we can define and study respective independent $\mathrm{N}$ - and/or P-structures on $\mathbf{T V}$, or $\mathbf{T}^{*} \mathbf{V}$.

The coefficients of d-connections can be defined in corresponding $\mathrm{N}$-adapted forms with respect to $\mathrm{N}$-adapted frames (11) and (12),

$$
\mathbf{D}_{\mathbf{e}_{\beta}} \mathbf{e}_{\gamma}:=\Gamma_{\beta \gamma}^{\alpha} \mathbf{e}_{\alpha} \text { and }{ }^{\prime} \mathbf{D}_{{ }_{1} \mathbf{e}_{\beta}}{ }^{\prime} \mathbf{e}_{\gamma}:={ }^{\prime} \Gamma_{\beta \gamma}^{\alpha}{ }^{\prime} \mathbf{e}_{\alpha},
$$

where (for a h-v splitting)

$$
\begin{aligned}
& \mathbf{D}_{\mathbf{e}_{k}} \mathbf{e}_{j}:=L^{i}{ }_{j k} \mathbf{e}_{i}, \mathbf{D}_{\mathbf{e}_{k}} e_{b}:=\dot{L}^{a}{ }_{b k} e_{a}, \\
& \mathbf{D}_{e_{c}} \mathbf{e}_{j}:=\dot{C}^{i}{ }_{j c} \mathbf{e}_{i}, \mathbf{D}_{e_{c}} e_{b}:=C_{b c}^{a} e_{a}
\end{aligned}
$$

and (for a h-cv splitting)

$$
\begin{aligned}
& { }^{\prime} \mathbf{D}_{{ }_{\mathbf{e}_{k}}}{ }^{\prime} \mathbf{e}_{j}:={ }^{\prime} L^{i}{ }_{j k}{ }^{\prime} \mathbf{e}_{i},{ }^{\prime} \mathbf{D}_{\mathbf{e}_{k}}{ }^{\prime} e^{b}:=-{ }^{\prime} L_{a k}^{b}{ }^{\prime} e^{a}, \\
& { }^{\prime} \mathbf{D}_{{ }^{\prime} e^{c}}{ }^{\prime} \mathbf{e}_{j}:={ }^{\prime} C^{i}{ }_{j}^{c}{ }^{\prime} \mathbf{e}_{i},{ }^{\prime} \mathbf{D}{ }^{c} e^{c}{ }^{\prime} e^{b}:=-{ }^{1} C_{a} b c{ }^{\prime} e^{a} .
\end{aligned}
$$

So, the N-adapted coefficients of d-connections on (co) tangent Lorentz bundles are respectively parameterized

$$
\begin{aligned}
& \Gamma_{\beta \gamma}^{\alpha}=\left\{L_{j k}^{i}, \dot{L}_{b k}^{a}, \dot{C}^{i}{ }_{j c}, C_{b c}^{a}\right\} \text { and } \\
& { }^{\prime} \Gamma_{\beta \gamma}^{\alpha}=\left\{{ }^{\prime} L_{j k}^{i},{ }^{\prime} L_{a k}^{b},{ }^{b} C^{i c}{ }_{j},{ }^{\prime} C_{a}^{b c}\right\} .
\end{aligned}
$$

Such values can be used for computations of $\mathrm{h}-$ and/or vsplitting, cv-splitting, of covariant derivatives

$\mathbf{D}=\left({ }_{h} \mathbf{D},{ }_{v} \mathbf{D}\right) \quad$ and $/$ or $\quad{ }^{\prime} \mathbf{D}=\left({ }_{h}^{1} \mathbf{D},{ }_{v}^{1} \mathbf{D}\right)$,

where ${ }_{h} \mathbf{D}=\left\{L^{i}{ }_{j k}, \dot{L}_{b k}^{a}\right\},{ }_{v} \mathbf{D}=\left\{\dot{C}^{i}{ }_{j c}, C_{b c}^{a}\right\}$ and ${ }_{h} \mathbf{D}=$ $\left\{{ }^{\prime} L_{j k}^{i},{ }^{\prime} L_{a k}^{b}\right\},{ }_{v}^{\prime} \mathbf{D}=\left\{{ }^{\prime} C^{i c}{ }_{j},{ }^{\prime} C_{a}^{b c}\right\}$.

Lemma 2.2 (Distortion of linear connection structures) Let us consider on TV a linear connection $\underline{D}$ (which is not obligatory a d-connection) and a d-connection D. Such values on $\mathbf{T}^{*} \mathbf{V}$, are respectively denoted ' $\underline{D}$ and ' $\mathbf{D}$ and can be related by corresponding distortion d-tensors

$\mathbf{Z}:=\mathbf{D}-\underline{D}$ and/or ${ }^{\prime} \mathbf{Z}:={ }^{\prime} \mathbf{D}-{ }^{\prime} \underline{D}$.

Proof Fixing respective $\mathrm{N}$-adapted frames, such distortion d-tensors

$$
\begin{aligned}
& \mathbf{Z}_{\beta \gamma}^{\alpha}=\Gamma_{\beta \gamma}^{\alpha}-\underline{\Gamma}_{\beta \gamma}^{\alpha} \\
& =\left\{Z_{j k}^{i}=L_{j k}^{i}-\underline{L}_{j k}^{i}, \dot{Z}_{b k}^{a}\right. \\
& =\dot{L}_{b k}^{a}-\underline{L}_{b k}^{a}, \dot{Z}^{i}{ }_{j c}=\dot{C}^{i}{ }_{j c}-\underline{C}_{j c}^{i}, Z_{b c}^{a} \\
& \left.=C_{b c}^{a}-\underline{C}_{b c}^{a}\right\} \text { and } \\
& { }^{\prime} \mathbf{Z}_{\beta \gamma}^{\alpha}={ }^{\prime} \boldsymbol{\Gamma}_{\beta \gamma}^{\alpha}-{ }^{\prime} \underline{\boldsymbol{\Gamma}}_{\beta \gamma}^{\alpha} \\
& =\left\{{ }^{\prime} Z^{i}{ }_{j k}={ }^{\prime} L_{j k}^{i}-{ }^{\prime} \underline{L}_{j k}^{i},{ }^{\prime} \dot{Z}_{a k}^{b}\right. \\
& ={ }^{\prime} \dot{L}_{a k}^{b}-{ }^{\prime} \underline{L}_{a k}^{b},{ }^{b} \dot{Z}^{i c}{ }_{j} \\
& \left.={ }^{\prime} \dot{C}^{i c}{ }_{j}^{c}-{ }^{\prime} \underline{C}^{i c}{ }_{j},{ }^{\prime} Z_{a}^{b c}={ }^{\prime} C_{a}^{b c}-{ }^{1} \underline{C}_{a}^{b c}\right\},
\end{aligned}
$$

can be constructed in explicit form by considering corresponding differences of $\mathrm{N}$-adapted coefficients for linear and d-connections.

Using similar definitions and theorems as for linear connections, we can prove for d-connections: 
Definition 2.6 -Theorem ${ }^{5}$ (Curvature, torsion and nonmetricity of d-connections) Any d-connection $\mathbf{D}$, or ' $\mathbf{D}$, is characterized by respective curvature $\left(\mathcal{R}\right.$, or $\left.{ }^{\prime} \mathcal{R}\right)$, torsion $\left(\mathcal{T}\right.$, or $\left.{ }^{\prime} \mathcal{T}\right)$, and nonmetricity, $(\mathcal{Q}$, or ' $\mathcal{Q})$, d-tensors defined and computed in standard forms:

$$
\begin{aligned}
& \mathcal{R}(\mathbf{X}, \mathbf{Y}):=\mathbf{D}_{\mathbf{X}} \mathbf{D}_{\mathbf{Y}}-\mathbf{D}_{\mathbf{Y}} \mathbf{D}_{\mathbf{X}}-\mathbf{D}_{[\mathbf{X}, \mathbf{Y}]}, \\
& \mathcal{T}(\mathbf{X}, \mathbf{Y}):=\mathbf{D}_{\mathbf{X}} \mathbf{Y}-\mathbf{D}_{\mathbf{Y}} \mathbf{X}-[\mathbf{X}, \mathbf{Y}] \text { and } \\
& \mathcal{Q}(\mathbf{X}):=\mathbf{D}_{\mathbf{X}} \mathbf{g} \\
& \text { or }{ }^{\prime} \mathcal{R}\left({ }^{\prime} \mathbf{X},{ }^{\prime} \mathbf{Y}\right):={ }^{\prime} \mathbf{D}_{{ } \mathbf{X}}{ }^{\prime} \mathbf{D}_{{ } \mathbf{Y}}-{ }^{\prime} \mathbf{D}_{{ } \mathbf{Y}}{ }^{\prime} \mathbf{D}_{\mid} \mathbf{X}-{ }^{\prime} \mathbf{D}_{\left[{ }_{[} \mathbf{X},{ }^{\prime} \mathbf{Y}\right]} \text {, } \\
& { }^{\prime} \mathcal{T}\left({ }^{\prime} \mathbf{X},{ }^{\prime} \mathbf{Y}\right):={ }^{\prime} \mathbf{D}_{\mid} \mathbf{X}{ }^{\mid} \mathbf{Y}-{ }^{\prime} \mathbf{D}{ }_{\mid} \mathbf{Y}{ }^{\prime} \mathbf{X}-\left[{ }^{\prime} \mathbf{X},{ }^{\prime} \mathbf{Y}\right] \text { and } \\
& { }^{\prime} \mathcal{Q}\left({ }^{\prime} \mathbf{X}\right):={ }^{\prime} \mathbf{D} \cdot \mathbf{X}{ }^{\prime} \mathbf{g} \text {. }
\end{aligned}
$$

The N-adapted coefficients for the curvature, torsion and nonmetricity d-tensors (29) are provided in Appendix, see Corollary A.1.

\subsubsection{The Ricci and Einstein d-tensors}

The Ricci tensor for a d-connection on a (co)tangent bundle can be constructed in standard form by contracting, for instance, the first and forth indices of respective curvature d-tensors $\mathcal{R}$ and/or ' $\mathcal{R}$ (29).

Definition 2.7 -Theorem (Ricci tensors for d-connections) The Ricci d-tensors are defined and computed as Ric = $\left\{\mathbf{R}_{\alpha \beta}:=\mathbf{R}_{\alpha \beta \tau}^{\tau}\right\}$, for a d-connection $\mathbf{D}$, and ' Ric $=\left\{{ }^{\prime} \mathbf{R}_{\alpha \beta}:=\right.$ ${ }^{\prime} \mathbf{R}_{\alpha \beta \tau}^{\tau}$ \}, for a d-connection ' $\mathbf{D}$.

In $\mathrm{N}$-adapted form using formulas (A.1), one proves

Corollary 2.5 (Computation of Ricci d-tensors) The $\mathrm{N}$ adapted coefficients of the Ricci d-tensors of a d-connection in a (co)tangent Lorentz bundle are parameterized in $h$ and/or $v$-, or cv-form, by formulas

$$
\begin{aligned}
\mathbf{R}_{\alpha \beta}= & \left\{R_{h j}:=R_{h j i}^{i}, \quad R_{j a}:=-P^{i}{ }_{j i a},\right. \\
& \left.R_{b k}:=P_{b k a}^{a}, R_{b c}=S_{b c a}^{a}\right\}, \quad \text { or } \\
{ }^{\prime} \mathbf{R}_{\alpha \beta}= & \left\{{ }^{1} R_{h j}:={ }^{\prime} R^{i}{ }_{h j i},{ }^{\prime} R_{j}^{a}:=-{ }^{\prime} P_{j i}^{i}{ }^{a},\right. \\
& \left.{ }^{\prime} R_{k}^{b}:={ }^{\prime} P_{a k}^{b}{ }^{a},{ }^{a} R^{b c}={ }^{\prime} S_{a}^{b c a}\right\} .
\end{aligned}
$$

If a (co)tangent bundle is enabled both with a d-connection, $\mathbf{D}$ (or 'D), and d-metric, g (13) (or ' $\mathbf{g}$ (14)), [in particular, we can consider canonical d-metrics $\widetilde{\mathbf{g}}$ (18) and/or ' $\widetilde{\mathbf{g}}(19)$ encoding MDRs], we can introduce nonholonomic Ricci scalars:

Definition 2.8 -Theorem (Scalar curvature of d-connection) The scalar curvature of a d-connection $\mathbf{D}$, or ' $\mathbf{D}$, can be defined and computed for the inverse d-metric $\mathbf{g}^{\alpha \beta}$, or ${ }^{\prime} \mathbf{g}^{\alpha \beta}$,

$$
{ }_{s} R:=\mathbf{g}^{\alpha \beta} \mathbf{R}_{\alpha \beta}=g^{i j} R_{i j}+g^{a b} R_{a b}=R+S, \quad \text { or }
$$

\footnotetext{
5 In mathematical physics, there are used terms like DefinitionTheorem/-Lemma/-Corollary etc. for such definitions (new ideas, concepts, or conventions) which motivated by certain explicit geometric constructions and/or requesting formulation of some theorems and respective mathematical proofs.
}

$$
{ }_{s}{ }^{1} R:={ }^{\prime} \mathbf{g}^{\alpha \beta}{ }^{\prime} \mathbf{R}_{\alpha \beta}={ }^{\prime} g^{i j}{ }^{\prime} R_{i j}+{ }^{\prime} g^{a b}{ }^{\prime} R_{a b}={ }^{\prime} R+{ }^{\prime} S,
$$

with respective h- and v-components $R=g^{i j} R_{i j}, S=$ $g^{a b} S_{a b}$, or ${ }^{\prime} R={ }^{\prime} g^{i j}{ }^{\prime} R_{i j},{ }^{\prime} S={ }^{\prime} g_{a b}{ }^{\prime} S^{a b}$.

Using above Definitions-Theorems and Corollaries, we formulate

Definition 2.9 -Theorem (the Einstein tensors for d-connections) By constructions, the Einstein d-tensors on TV and/or $\mathbf{T}^{*} \mathbf{V}$ are defined:

$$
\begin{aligned}
& E n=\left\{\mathbf{E}_{\alpha \beta}:=\mathbf{R}_{\alpha \beta}-\frac{1}{2} \mathbf{g}_{\alpha \beta}{ }_{s} R\right\} \text { and/or } \\
& { }^{'} E n=\left\{{ }^{\prime} \mathbf{E}_{\alpha \beta}:={ }^{\prime} \mathbf{R}_{\alpha \beta}-\frac{1}{2}{ }^{\prime} \mathbf{g}_{\alpha \beta}{ }_{s}^{\prime} R\right\} .
\end{aligned}
$$

Proof Such proofs follow from explicit constructions on regions of some atlases covering respectively $\mathbf{T V}$ and/or $\mathbf{T}^{*} \mathbf{V}$ using N-adapted coefficients (A.1) and (30) and/or (31).

It should be noted that nonholonomic Einstein-Cartan models on (co)tangent Lorentz bundles can be elaborated for respective data (g, D) and/or ( ' $\mathbf{g}$, 'D) considering, for instance, generalized spinning liquid models for effective matter as sources of d-torsions $\mathcal{T}$ and ${ }^{\prime} \mathcal{T}$, see (A.2) and imposing the metricity conditions. How to formulate such nonholonomic Riemann-Cartan and metric-affine and generalized Lagrange-Hamilton-Finsler theories with nontrivial nonmetricities $\mathcal{Q}$ and/or ' $\mathcal{Q}$, see formulas (A.3), was studied in monograph [169]. ${ }^{6}$ For $\mathbf{g}=\widetilde{\mathbf{g}}$ and/or ${ }^{\prime} \mathbf{g}={ }^{\prime} \widetilde{\mathbf{g}}$ determined by MDRs, we can model generalized Lagrange-Finsler and/or Hamilton-Cartan phase spaces with nonmetric backgrounds extending the class of (co)Finsler geometries with the Chern and/or Berwald type connections [16,20,21,124,125]. Such geometric models result in a number of ambiguities for constructing physically self-consistent theories of locally anisotropic interactions for gravitational, gauge, scalar and spinor fields (and further (super) string/noncommutative, quantum etc. generalizations) as it is concluded in Refs. [169, 175, 185, 193,194].

An axiomatic approach to Finsler like generalizations of GR on (co)tangent Lorentz bundles [187,194] can be formulated for theories when a triple consisting from a $\mathrm{N}$-connection, a d-metric (or almost symplectic) and a dconnection structures are canonically and metric compatible determined (following certain minimal geometric and physical principles) by a fundamental generating Lagrange and/or Hamilton function. In next subsections, we construct and study some canonical and physically important d-connection structures which allows us to elaborate physically viable classical and quantum models of locally anisotropic gravitational

\footnotetext{
${ }^{6}$ See Chapter 1 and references therein related to Lagrange-HamiltonFinsler generalizations of the metric-affine gravity elaborated by F. W. Hehl's group.
} 
and matter field interactions. Such theories encode respective MDRs of type (1), with a fixed point on a base spacetime manifold, or for a generalized phase spacetime modelled on a (co)tangent Lorentz bundle.

\subsubsection{Physically important (Filsler like) d-connections on (co)tangent bundles}

For elaborating MGTs and geometric mechanics models, one considers more specials classes of d-connections on which can be defined completely by a d-metric/almost symplectic structure determined by a respective Lagrange-Finsler and/or Hamilton-Cartan fundamental form.

Definition 2.10 -Theorem (Physically important d-connections) The Almost Kähler-Lagrange and/or almost Kä hlerHamilton phase spaces (determined, or not, by respective $\operatorname{MDRs}(1)$ and a possible $\mathcal{L}$-duality) are characterized respectively by such geometric and physically important linear connections and canonical/almost symplectic connections:
- The definition and proofs of existence of $\widehat{\mathbf{D}}$ and $\widehat{\mathbf{D}}$ are provided by globalizing the constructions from Corollary A.2 and respective $\mathrm{N}$-adapted coefficients (A.4) and/or (A.4). Such canonical d-connections play a crucial role for decoupling and solving in general off-diagonal forms for various types of Finsler like d-connections of gravitational and matter filed like equations in MGTs, i.e. for elaborating the AFDM.

- The fundamental Lagrange-Finsler generating functions (which may be, or not, induced by MDRs and LIVs) can be used for modeling almost Kähler models of generalized Lagrange and/or Hamilton spaces. In relativistic form, such theories were elaborated and applied to deformation quantization of gravity theories and possible noncommutative generalizations in Refs. $[8,12,161$, $172,174,177,184,197,204,206]$, where there are provided necessary proofs and $\mathrm{N}$-adapted coefficients for the almost symplectic connections $\widetilde{\mathbf{D}}$ and ' $\widetilde{\mathbf{D}}$. There are not considered such models and solutions in this work (even

$$
\begin{aligned}
{[\mathbf{g}, \mathbf{N}] } & \simeq[\widetilde{\mathbf{g}}, \widetilde{\mathbf{N}}] \simeq[\widetilde{\theta}:=\widetilde{\mathbf{g}}(\widetilde{\mathbf{J}} \cdot, \cdot), \widetilde{\mathbf{P}}, \widetilde{\mathbf{J}}, \widetilde{\mathbb{J}}] \\
& \Longrightarrow\left\{\begin{array}{l}
\nabla: \quad \nabla \mathbf{g}=0 ; \mathbf{T}[\nabla]=0 \\
\widehat{\mathbf{D}}: \widehat{\mathbf{D}} \mathbf{g}=0 ; h \widehat{\mathbf{T}}=0, v \widehat{\mathbf{T}}=0 . \\
\widetilde{\mathbf{D}}: \quad \widetilde{\mathbf{D}} \widetilde{\theta}=0, \widetilde{\mathbf{D}} \widetilde{\theta}=0
\end{array}\right.
\end{aligned}
$$

\section{Lagrange LC-connection;}

canonical Lagrange d-connection;

almost symplectic Lagrange d-connection.;

and/or

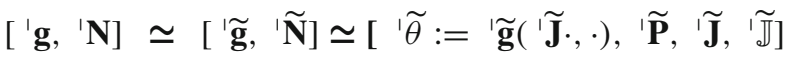

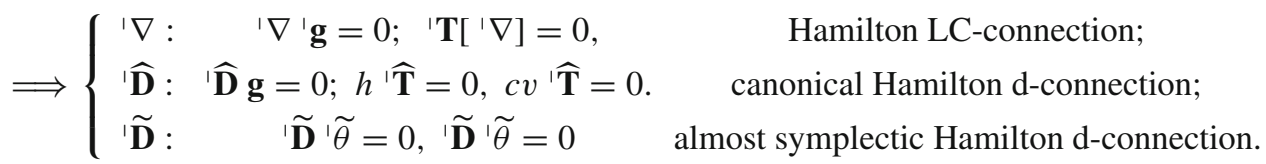

Proof It is sketched step by step by a respective linear connection/d-connection determined by the same fundamental geometric objects up to frame transforms:

- Both variants of LC-connections on (co)tangent bundles, $\nabla$ and/or $\quad \nabla$, are defined and constructed in standard abstract, coordinate, or $\mathrm{N}$-adapted forms using (respectively) $\mathbf{g}$ (13) and ' $\mathbf{g}$ (14). We have to consider $\widetilde{\mathbf{g}}$ (18) and ' $\widetilde{\mathbf{g}}(19)$ if we work in not $\mathrm{N}$-adapted form with generic off-diagonal metrics of type (20). Here, we note that LCconnections can be defined without $\mathrm{N}$-connection structures, i.e. such linear connections are not d-connections. Nevertheless, such values may encode Lagrange and/or Hamilton structures if they are computed for metrics/dmetrics encoding, for instance, MDRs and respective Hessians (modelling Lagrange and/or Hamilton spaces, see (8) and/or (6)).
$\widetilde{\mathbf{D}}$ was used for finding commutative and noncommutative Finsler black hole solutions $[183,189,196])$. It is important to analyze formulas with $\widetilde{\mathbf{D}}$ and ' $\widetilde{\mathbf{D}}$ because such dconnections transform into standard Finsler-Cartan ones for respective nonholonomic parameterizations of the generating functions, Sasaki type induced d-metrics and canonical N-connection structures.

It is well-known Chern's definition [17,41] that Finsler geometry is an example of geometry when the assumption on quadratic linear elements is dropped and (we emphasize additionally) there are elaborated new geometric constructions determined by nonlinear quadratic line elements. Various mathematical approaches were developed for respective Finsler generalized norms and metric structures. We need additional assumptions and have to performed more sophis- 
ticate geometric constructions involving $\mathbf{N}$-connection and d-connection structures in order to formulate self-consistent and physically viable Finsler like generalizations of Einstein gravity for theories with MDRs and LIVs.

Remark 2.7 (Complete and self-consistent models of Finsler geometry) The first self-consistent model of Finsler geometry (with local geometric constructions with generalized metric, $\mathrm{N}$-connection and d-connection structures, and associated Nframes) was elaborated before 1935 by E. Cartan [27] and citations therein. In those works, there were used coordinate transforms of nonlinear and linear connections and developed the original constructions with nonlinear quadratic elements due to the famous habilitation thesis of B. Riemann defended in 1854, see [115]; and introduced the term of Finsler geometry using the original work [46]. Conventionally, that model of Finsler-Cartan geometry, which is metric compatible, can be described on tangent bundles (or on manifolds with fibred structure) by a triple of fundamental geometric structures $(F: \widetilde{\mathbf{g}}, \widetilde{\mathbf{N}}, \widetilde{\mathbf{D}})$ all determined by a so-called Finsler metric (generating function) $F$ which can be associated to a class of MDRs and LIVs subjected to certain homogeneity conditions.

One of the most important nontrivial characteristics of a Finsler geometry model is that it posses, in general, a nontrivial nonholonomic structure determined by a $\mathrm{N}$-connection structure. Even for certain additional geometric/physical assumptions, all geometric objects on a Finsler space can be determined by a $F(x, y)$ on a tangent bundles, such a theory is with a triple of fundamental geometric objects. The geometric and physical models related to (generalized) Finsler theories are very different from the (pseudo) Riemann geometry which is completely determined by the metric structure.

\subsubsection{Distortion tensors for connections and curvature and Ricci tensors}

MGTs with MDRs on (co)tangent bundles are characterized by multi-connection structures which, in principle, can be derived by a metric structure (induced by a fundamental is most convenient to work with the canonical d-connections $\widehat{\mathbf{D}}$ and $\widehat{\mathbf{D}}$. Following the conditions of Lemma 2.2, we prove

Theorem 2.5 (Existence of unique and physically important distortions of connections) There are unique distortions relations

$$
\begin{aligned}
& \widehat{\mathbf{D}}=\nabla+\widehat{\mathbf{Z}}, \widetilde{\mathbf{D}}=\nabla+\widetilde{\mathbf{Z}} \text {, and } \\
& \widehat{\mathbf{D}}=\widetilde{\mathbf{D}}+\mathbf{Z}, \text { determined by }(\mathbf{g}, \mathbf{N}) ; \\
& \widehat{\widehat{\mathbf{D}}}={ }^{\prime} \nabla+\widehat{\mathbf{Z}},{ }^{\prime} \widetilde{\mathbf{D}}={ }^{\prime} \nabla+{ }^{\prime} \widetilde{\mathbf{Z}}, \text { and } \\
& \widehat{\mathbf{D}}={ }^{\prime} \widetilde{\mathbf{D}}+{ }^{'} \mathbf{Z}, \text { determined by }\left({ }^{\prime} \mathbf{g},{ }^{\prime} \mathbf{N}\right) ;
\end{aligned}
$$

for distortion d-tensors $\widehat{\mathbf{Z}}, \widetilde{\mathbf{Z}}$, and $\mathbf{Z}$, on $T \mathbf{T V}$, and $\widehat{\mathbf{Z}}, \quad \widetilde{\mathbf{Z}}$, and ${ }^{\prime} \mathbf{Z}$, on $T \mathbf{T}^{*} \mathbf{V}$.

It should be noted that the d-tensor $\widehat{\mathbf{Z}}$ in above formulas is an algebraic combination of coefficients $\widehat{\mathbf{T}}_{\alpha \beta}^{\gamma}[\mathbf{g}, \mathbf{N}] \mathrm{com}$ puted by introducing formulas (A.4) into (A.2) (similarly for cotangent bundles). Such values can be induced by corresponding nonholonomic structures (23) and (24) determined by MDRs. This proves:

Consequence 2.2 MDRs (1) are characterized by respective canonical and/or almost symplectic distortion d-tensors $\widehat{\mathbf{Z}}[\widetilde{\mathbf{g}}, \widetilde{\mathbf{N}}], \widetilde{\mathbf{Z}}[\widetilde{\mathbf{g}}, \widetilde{\mathbf{N}}]$, and $\mathbf{Z}[\widetilde{\mathbf{g}}, \widetilde{\mathbf{N}}]$, for (almost symplectic)

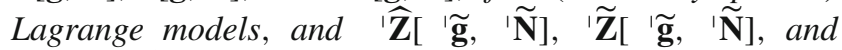
'Z[ ' $\widetilde{\mathbf{g}}$, ' $\widetilde{\mathbf{N}}]$, for (almost symplectic) Hamilton models.

Using nonholonomic frame transforms and distortions, we can elaborate equivalent models of phase spaces and MGTs formulated in terms of "preferred" for certain purposes geometric data. In the geometric "language" of "tilde" objects, we obtain relativistic mechanical like formulations for the geometry of phase spaces (locally anisotropic ether) with straightforward procedures for performing deformation quantization. For "hat" objects, we obtain many possibilities for decoupling and integrating physically important systems of nonlinear PDEs.

Conclusion 2.4 (Equivalent canonical geometric data for modeling phase spaces with MDRs) The phase space geom-

\begin{tabular}{|c|c|c|c|}
\hline & $(\mathbf{g}, \mathbf{N}, \widehat{\mathbf{D}}) \leftrightarrows(L: \widetilde{\mathbf{g}}, \widetilde{\mathbf{N}}, \widetilde{\mathbf{D}})$ & $\widetilde{\mathbf{D}})$ & $\leftrightarrow[(\mathbf{g}[N]$, \\
\hline $\begin{array}{l}\text { dicator } \varpi \\
\text { see }(1)\end{array}$ & $\begin{array}{l}\uparrow \text { possible } \mathcal{L} \text {-duality } \& \\
' \mathbf{N}, \widehat{\mathbf{D}}) \leftrightarrows(H: ' \widetilde{\mathbf{g}} . ' \widetilde{\mathbf{N}}\end{array}$ & $\begin{array}{l}\text { symplectomorphisms }[8] \\
\leftrightarrow(' \widetilde{\theta}, \widetilde{\mathbf{P}}, \quad, \widetilde{\mathbf{J}}, \widetilde{\mathbb{J}}, \quad, \widetilde{\mathbf{D}})\end{array}$ & $\begin{array}{l}\uparrow \text { not } N \text {-adapt } \\
\left.\left({ }^{\prime} \mathbf{g}\left[{ }^{\prime} N\right],{ }^{\prime} \nabla\right)\right],\end{array}$ \\
\hline
\end{tabular}
etry can be described in equivalent forms (up to respective nonholonomic deformations of the linear connection structures and nonholonomic frame transforms) by such data

In brief, we shall say that certain geometric construc-

Lagrange-Hamilton function) as we proved in DefinitionTheorem 2.10. One fixes a (non)linear connection structure following certain physical/geometric principles resulting in a self-consistent and experimentally verified physical theory. In order to construct exact solutions following the AFDM, it tions are canonical (i.e. formulated in canonical nonholonomic variables) if they are performed for "hat", or "tilde", dconnections and related geometric objects uniquely derived for certain Lagrange-Hamilton fundamental generating functions (in particular, for a Finsler metric $F$ ). 
Convention 2.1 (Existence of a preferred d-connection for decoupling (modified) Einstein equations and generating offdiagonal solutions) We can work with canonical d-connection structures on (co)tangent bundles, $\widehat{\mathbf{D}}$ and/or ' $\widehat{\mathbf{D}}$ which allows us to decouple and integrate in most general exact and parametric forms (with generic off-diagonal metrics and generalized connections and effective matter sources depending, in principle, on all spacetime and phase space coordinates) the gravitational and matter field equations in MGTs and GR, see details and proofs for above presented references for the AFDM (in the proof of Definition-Theorem 2.10) and next sections.

Lagrange-Finsler variables can be introduced on 4-d, and higher dimension, (pseudo) Riemann spaces and in GR, see details and a number of examples in Refs. $[68,70,149,150$, $152,153,158,161,169,171,173,186,188]$.

Remark 2.8 (Lagrange-Hamilton variables in Einstein gravity, GR) Prescribing a generating function $L\left(x^{1}, x^{2}, y^{3}, y^{4}=\right.$ $t$ ), [in this remark, $\alpha=(i, a)$, for $i=1,2$ and $a=$ $3,4]$ on a Lorentz manifold $\left({ }^{4} V, \mathbf{g}_{\alpha \beta}\right), \operatorname{dim}\left({ }^{4} V\right)$, endowed with a metric of local signature $(+++-)$ and conventional splitting, we can construct a canonical $\mathrm{N}$-connection ${ }^{2+2} \widetilde{\mathbf{N}}: T\left({ }^{4} V\right)=h\left({ }^{4} V\right) \oplus v\left({ }^{4} V\right)$, see Theorem 2.2. Respectively, it is defined an $\mathrm{N}$-adapted frame structure with $2+2$ splitting of type $\widetilde{\mathbf{e}}_{\alpha}=\left(\widetilde{\mathbf{e}}_{1}, \widetilde{\mathbf{e}}_{2}, e_{3}, e_{4}\right)$, see (16). Via frame/coordinate transforms, we can express the pseudoRiemannian metric as a canonical d-metric, $\mathbf{g}_{\alpha \beta} \simeq \widetilde{\mathbf{g}}_{\alpha \beta}$ (18), and/or in off-diagonal form (20). Following conditions (32), we can construct in unique form three types of linear connections $\nabla[\mathbf{g} \simeq \widetilde{\mathbf{g}}], \widehat{\mathbf{D}}[\mathbf{g} \simeq \widetilde{\mathbf{g}}]$ and/o $\widetilde{\mathbf{D}}[\mathbf{g} \simeq \widetilde{\mathbf{g}}]$. This way, the pseudo-Riemannian geometry can be formulated equivalently in standard form with data $(\mathbf{g}, \nabla)$, and/or in LagrangeFinsler like variables $(\widetilde{\mathbf{g}}, \widehat{\mathbf{N}}, \widehat{\mathbf{D}})$ and/or $(\widetilde{\mathbf{g}}, \widetilde{\mathbf{N}}, \widetilde{\mathbf{D}})$, with respective distortion relations, $\widehat{\mathbf{D}}=\nabla+\widehat{\mathbf{Z}}$ and $\widetilde{\mathbf{D}}=\nabla+\widetilde{\mathbf{Z}}$. Such geometric models (we can say "toy" Lagrange-Finsler models on manifolds with conventional nonholonomic $2+2$ splitting) are elaborated for (pseudo) Riemannian manifolds with prescribed nonholonomic fibered structure. Fibered splitting is of type $h\left({ }^{4} V\right) \oplus v\left({ }^{4} V\right)$ but the "standard" LagrangeFinsler geometrise are constructed for $T T V=h T V \oplus v T V$.

An important example is that when imposing certain (in general, nonholonomic) constraints of type $\widehat{\mathbf{Z}}=0$, we obtain $\widehat{\mathbf{D}}_{\widehat{\mid}=0} \simeq \nabla$ even $\widehat{\mathbf{D}} \neq \nabla .^{7}$ If such conditions are satisfied, we can extract (pseudo) Riemannian LC-configurations from more (general) nonholonomic metric-affine structures.

\footnotetext{
$\overline{7}$ The frame/coordinate transformation laws of nonlinear and distinguished/linear connections are different from that of tensors. It is possible to define such a frame structure when different connections may be determined by the same set of coefficients with respect to such a special frame and by different sets in other systems of reference.
}

Corollary 2.6 (Extracting LC-configurations by additional (non)holonomic constraints) One extracts LC-configurations from $\widehat{\mathbf{D}}$ and/or ' $\widehat{\mathbf{D}}$ for respective zero distortions, $\widehat{\mathbf{Z}}$ and/or $\widehat{\mathbf{Z}}$, if there are imposed zero torsion conditions for $\widehat{\mathcal{T}}=$ $\left\{\widehat{\mathbf{T}}_{\alpha \beta}^{\gamma}\right\}=0$ and/or $\quad ' \widehat{\mathcal{T}}=\left\{{ }^{\gamma} \widehat{\mathbf{T}}_{\alpha \beta}^{\gamma}\right\}=0$, see (A.2). Such conditions are satisfied if

$$
\begin{aligned}
\widehat{C}_{j b}^{i} & =0, \Omega^{a}{ }_{j i}=0 \text { and } \widehat{L}_{a j}^{c}=e_{a}\left(N_{j}^{c}\right) ; \\
{ }^{\prime} \widehat{C}_{j}^{i b} & =0,{ }^{\prime} \Omega_{a j i}=0 \text { and }{ }^{\prime} \widehat{L}_{c j}^{a}={ }^{\prime} e^{a}\left({ }^{\prime} N_{c j}\right) .
\end{aligned}
$$

Proof Let us sketch such a proof on TV (the constructions on $\mathbf{T}^{*} \mathbf{V}$ are similar). Introducing (32) in (A.2), we can check that one obtains zero values for

$$
\begin{aligned}
& \widehat{T}^{i}{ }_{j k}=\widehat{L}_{j k}^{i}-\widehat{L}_{k j}^{i}, \widehat{T}^{i}{ }_{j a}=\widehat{C}^{i}{ }_{j b}, \widehat{T}^{a}{ }_{j i}=-\Omega^{a}{ }_{j i}, \\
& \widehat{T}_{a j}^{c}=\widehat{L}_{a j}^{c}-e_{a}\left(N_{j}^{c}\right), \widehat{T}_{b c}^{a}=\widehat{C}_{b c}^{a}-\widehat{C}_{c b}^{a} .
\end{aligned}
$$

if the conditions (36) are satisfied.

In a series of works $[15,79,133,175,186,187,194,202]$, we proved that the equations (36) can be solved in explicit form for manifolds/bundle spaces of dimensions 4-10. Similarly, the equations (37) can be integrated on $\mathbf{T}^{*} \mathbf{V}$.

Introducing distortions from Theorem 2.5 into formulas (29), we can prove in abstract and $\mathrm{N}$-adapted forms:

Theorem 2.6 (Existence of canonical distortions of Riemannian and Ricci d-tensors determined by MDRs) There are canonical distortion relations encoding MDRs for respective Lagrange-Finsler nonholonomic variables:

- For the curvature d-tensors,

$$
\begin{aligned}
\widehat{\mathcal{R}}[\mathbf{g}, \widehat{\mathbf{D}} & =\nabla+\widehat{\mathbf{Z}}]=\mathcal{R}[\mathbf{g}, \nabla]+\widehat{\mathcal{Z}}[\mathbf{g}, \widehat{\mathbf{Z}}], \\
{ }^{\prime} \widehat{\mathcal{R}}\left[{ }^{\prime} \mathbf{g},{ }^{\prime} \widehat{\mathbf{D}}\right. & \left.={ }^{\prime} \nabla+{ }^{\prime} \widehat{\mathbf{Z}}\right]={ }^{\prime} \mathcal{R}\left[{ }^{\prime} \mathbf{g},{ }^{\prime} \nabla\right]+{ }^{\prime} \widehat{\mathcal{Z}}\left[{ }^{\prime} \mathbf{g},{ }^{\prime} \widehat{\mathbf{Z}}\right],
\end{aligned}
$$

with respective distortion d-tensors $\widehat{\mathcal{Z}}$, on $\mathbf{T V}$, and ' $\widehat{\mathcal{Z}}$, on $\mathbf{T}^{*} \mathbf{V}$;

- For the Ricci d-tensors,

$$
\begin{aligned}
\widehat{R} i c[\mathbf{g}, \widehat{\mathbf{D}} & =\nabla+\widehat{\mathbf{Z}}]=\operatorname{Ric}[\mathbf{g}, \nabla]+\widehat{Z} i c[\mathbf{g}, \widehat{\mathbf{Z}}], \\
{ }^{\prime} \widehat{\operatorname{R}} i c\left[{ }^{\prime} \mathbf{g},{ }^{\prime} \mathbf{D}\right. & \left.={ }^{\prime} \nabla+{ }^{\mid} \widehat{\mathbf{Z}}\right] \\
& ={ }^{\prime} \operatorname{Ric}\left[{ }^{\prime} \mathbf{g},{ }^{\prime} \nabla\right]+{ }^{'} \widehat{Z} i c\left[{ }^{\prime} \mathbf{g},{ }^{\widehat{\mathbf{Z}}}\right],
\end{aligned}
$$

with respective distortion d-tensors $\widehat{Z} i c$, on $\mathbf{T V}$, and $\widehat{Z}$ ic , on $\mathbf{T}^{*} \mathbf{V}$;

- For the scalar curvature of canonical d-connection $\widehat{\mathbf{D}}$, or $\widehat{\mathbf{D}}$,

$$
\begin{aligned}
{ }_{s} \widehat{R}[\mathbf{g}, \widehat{\mathbf{D}} & =\nabla+\widehat{\mathbf{Z}}]=\mathcal{R}[\mathbf{g}, \nabla]+{ }_{s} \widehat{Z}[\mathbf{g}, \widehat{\mathbf{Z}}], \\
{ }_{s} \widehat{R}\left[{ }^{\prime} \mathbf{g},{ }^{\prime} \widehat{\mathbf{D}}\right. & \left.={ }^{\prime} \nabla+\widehat{\mathbf{Z}}\right]={ }_{s} R\left[{ }^{\prime} \mathbf{g},{ }^{\prime} \nabla\right]+{ }_{s} \widehat{Z}\left[{ }^{\prime} \mathbf{g},{ }^{\prime} \widehat{\mathbf{Z}}\right],
\end{aligned}
$$

with respective distortion scalar functionals ${ }_{s} \widehat{Z}$, on $\mathbf{T V}$, and ${ }_{s}^{1} \widehat{Z}$, on $\mathbf{T}^{*} \mathbf{V}$. 
Proof We omit such tedious abstract and/or frame computations in this work. In $[9,68,69,164,165,168,169,182,183$, 186,188-190,196,198,199,201-203], there are provided details of similar proofs for Lagrange-Finsler spaces and on (pseudo) Riemannian manifolds with nonholonomic fibered structure. In this work, we shall construct exact solutions for MGTs encoding MDRs working with the canonical d-connection $\widehat{\mathbf{D}}$, or $\widehat{\mathbf{D}}$, which can be restricted to LCconfigurations by solving, respectively, the equations (36), or (37).

Remark 2.9 (MDR-distortions of canonical almost symplectic Lagrange-Hamilton structures) The conditions of above Theorem can be reformulated for distortions of the almost symplectic Lagrange, or Finsler, d-connections, for instance, considering

$$
\begin{aligned}
& \widetilde{\mathcal{R}}[\widetilde{\mathbf{g}} \simeq \widetilde{\theta}, \widetilde{\mathbf{D}}=\nabla+\widetilde{\mathbf{Z}}]=\mathcal{R}[\widetilde{\mathbf{g}} \simeq \widetilde{\theta}, \nabla]+\widetilde{\mathcal{Z}}[\widetilde{\mathbf{g}} \simeq \widetilde{\theta}, \widetilde{\mathbf{Z}}], \\
& { }^{\prime} \widetilde{\mathcal{R}}\left[{ }^{\prime} \widetilde{\mathbf{g}} \simeq{ }^{\prime} \tilde{\theta},{ }^{\prime} \widetilde{\mathbf{D}}={ }^{\prime} \nabla+{ }^{\prime} \widetilde{\mathbf{Z}}\right] \\
& ={ }^{\prime} \mathcal{R}\left[{ }^{\prime} \tilde{\mathbf{g}} \simeq{ }^{\prime} \tilde{\theta},{ }^{\prime} \nabla\right]+{ }^{\prime} \widetilde{\mathcal{Z}}\left[{ }^{\prime} \mathbf{g} \simeq{ }^{\prime} \widetilde{\theta},{ }^{\prime} \widetilde{\mathbf{Z}}\right],
\end{aligned}
$$

and any similar geometric objects with "tilde" symbols. Similar distortions can be defined and computed, for instance, for the Chern d-connection, Berwald d-connection and any d-connection structure which can be constructed in unique forms by the same d-metric and $\mathrm{N}$-connection structures [193]. Working with "hat" distortions stated in Theorem 2.6, we can prove the decoupling property of modified/generalized Einstein equations on $\mathbf{T V}$ and $\mathbf{T}^{*} \mathbf{V}$.

\subsubsection{On Akbar-Zadeh definition of the Ricci tensor for Finsler like spaces}

In a series of geometric models, it is applied an alternative concept of Ricci tensor for Finsler spaces which by definition does not involve the concept of $\mathrm{N}$-connection and/or d-connection, see [1,2]. Following Akbar-Zadeh constructions for a base manifold $M$, it in used as the curvature for Finsler geometry a value

$\breve{\mathbf{R}}=\left.\breve{R}_{k}^{i} d x^{k} \otimes \frac{\partial}{\partial x^{i}}\right|_{x}: T_{x} M \rightarrow T_{x} M$,

defined for any point $x \in M$. This type of "curvature" is not with an associated 2-form for a linear connection like in (29), but constructed directly from the data for nonlinear geodesic equations (15) and respective semi-spray $\tilde{G}^{k}$, when

$\breve{R}_{k}^{i}:=2 \frac{\partial \tilde{G}^{i}}{\partial x^{k}}-y^{j} \frac{\partial^{2} \tilde{G}^{i}}{\partial x^{j} \partial y^{k}}+2 \tilde{G}^{j} \frac{\partial^{2} \tilde{G}^{i}}{\partial y^{j} \partial y^{k}}-\frac{\partial \tilde{G}^{i}}{\partial y^{j}} \frac{\partial \tilde{G}^{j}}{\partial y^{k}}$.

Contracting indices in this formula, we can define and compute a scalar function $\breve{R}(x, y):=F^{-2} \breve{R}_{i}^{i}$. By definition, the scalar $\breve{R}$ is positive homogeneous of degree 0 in $v$-variables $y^{a}$. Such values are convenient for constructing and studies of geometric objects in $T_{x} M$ for a point $x \in M$.
The Ricci tensor "a la Akbar-Zadeh" is introduced by

$\breve{R} i c_{j k}:=F^{-2} \frac{\partial^{2} \breve{R}}{\partial y^{j} \partial y^{k}}$,

which is different from the (30) and other types of Ricci d-tensors constructed in explicit form, for instance, using the Finsler-Cartan d-connection. This geometric object is induced by the Finsler metric $F$ via inverse Hessian $\tilde{g}^{i j}$ and $\tilde{G}^{k}$. In $\breve{R} i c_{j k}$ (38), there are not involved definitions for a $\mathrm{N}$-connection structure, lifts of metrics on total space tangent bundle, and d-connections. For similar construction on a Lorentz manifold, $M \rightarrow \mathbf{V}$, such values may characterize certain MDRs (1) for $\tilde{g}_{i j}$ encoding such modifications. It is possible also to introduce a variant of Einstein like equation, $\breve{R} i c_{j k}=\lambda(x) \tilde{g}_{j k}$, i.e. when the scalar function $\breve{R}(x, y)=\lambda(x)$ is a function only on $h$-variables $x^{k}$. Another important property of $\breve{R} i c_{j k}$ (38) is that it is always symmetric (by definition) which provides a "simplified" model to study Ricci fields and/or evolution dynamics in any point $T_{x} M$, see [17].

Nevertheless, we consider that is not possible to define a complete, self-consistent and physically viable Finsler like MGT on $\mathbf{T V}$ and/or $\mathbf{T}^{*} \mathbf{V}$ working only with geometric objects generated by fundamental metric structures and do not involving additional constructions with $\mathrm{N}$-connection and d-connection structures. In order to introduce matter fields (tensor and spinor ones) and define geometric flows (with Perelman's functionals), we need covariant and local derivatives which are positively defined by some (non)linear connection objects, see critics in Refs. [185,193,194]. We can distinguish in physical theories certain configurations with $\breve{R} i c_{j k}$ for the h-components of a distortion relation like in Theorem 2.6 computing distortion relations, for instance,

$\widehat{R} i c_{i k}[\tilde{\mathbf{g}}, \widehat{\mathbf{D}}]=\breve{R} i c_{j k}\left[\tilde{g}_{i j}\right]+\breve{Z} i c_{i j}[\tilde{\mathbf{g}}, \widehat{\mathbf{Z}}]$.

Such constructions can not be complete without additional assumptions on $v$ - and/or $c v$-components and it is not possible to integrate in certain general forms modified Einstein equations with $\breve{R} i c_{j k}$ and/or $\breve{Z} i c$. In our works, we shall work with Ricci tensors for d-connections, of type (30), which allow certain nonholonomic transforms and constraints on $h$ subspaces in order to reproduce geometric structures related to $\breve{R} i c_{j k}$.

\section{Geometric and physical principles for gravity theories on (co)tangent bundles}

In this section, we formulate and analyse a set of geometric and physical principles which are necessary for selfconsistent causal formulations of MGTs defined by (generalized) Finsler multi-connection and/or bi-metric structures. For metric compatible d-connections, the geometric 
constructions are similar to the GR theory but extended on nonholonomic phase space manifolds or (co)tangent bundles. Physically important Lagrange densities are postulated as in the Einstein gravity theory but in terms of respective data with triples of fundamental geometric objects (i.e. some metric compatible d-metric, d-connection, and N-connection structures) on base Lorentz manifolds and their total (co)tangent bundles. Applying formal geometric and/or N-adapted variational methods, we derive formulas for (effective) sources of matter fields on curve phase spacetimes. There are constructed and studied explicit models with MDRs and locally anisotropic gauge and Higgs field interactions, modified massive and bi-metric theories, short-range models with LIVs.

\subsection{Principles for extending GR to \\ Finsler-Lagrange-Hamilton gravity theories}

The concept of flat Minkowski spacetime (with pseudoEuclidean signature) and the postulates which are necessary for formulating the special relativity theory, SRT, allow us to unify in a relativistic manner the classical mechanics and the Maxwell electromagnetic field theory. The approach was formulated in agreement with various types of MichelsonMorley experiments proving the existence of a constant maximal speed of light. Such logical and explicit experiments prove and involve certain fundamental properties of local spacetime local isotropy and homogeneity under the assumption that the concept of ether is not necessary for describing vacuum configurations. The most important symmetries in SRT are those of Lorentz (pseudo-rotation) and Poincaré (with additional translations) invariance with respect to linear group transforms. Such groups of automorphism of the Minkowski spacetime determine the conservation laws for energy and momentum (rotation momentum) values.

The GR theory was formulated in a standard (pseudo) Riemannian form using geometric data $(g, \nabla)$ for a Lorentz manifold $\mathbf{V}$ with causality structure. That approach preserves locally the symmetries of SRT following certain fundamental principles and axioms which can be extended for a large class of generalized theories with MDRs and LIVs. In this subsection, we speculate on modifications of GR for metric compatible Finsler like connections on $\mathbf{T V}$ and $\mathbf{T}^{*} \mathbf{V}$ of the principle of equivalence, with a partial realisation of the Mach principle; and of the general covariance principle. It is discussed the relation of equations of motion and conservation laws to Bianchi relations and nonholonomic deformations of d-connections. We elaborate on equivalent geometric and variational formulations in $\mathrm{N}$-adapted variables of generalized Einstein equations for MGTs with MDRs.

\subsubsection{Modified equivalence principles}

The experimental data show that the Newtonian gravitational force on a body is proportional to its inertial mass. This supports a fundamental idea that all bodies and fields are influenced in a similar "manner" by gravity and, indeed, point masses, light and small perturbations of scalar fields fall precisely in the same way in gravitational fields. Considering that motion of probing bodies and linearized interactions of classical fields are independent of the nature of the bodies, the paths of freely falling bodies define a preferred set of lines on a curved spacetime which locally is just as in SRT.

The world lines of freely falling bodies and small perturbations of electromagnetic/scalar fields in a gravitational field in GR are simply described by the geodesics of the (curved) spacetime metric. This suggests the possibility of ascribing the properties of the gravitational field to the structure of spacetime itself. Because MDRs on a Minkowski spacetime can be associated with metrics of type $g_{i j}(y)$ and/or $g_{i j}(p)$, when GR is defined by metrics of type $g_{i j}(x)$, we suppose that one can be formulated a generalized equivalence principle on some generalized Finsler spacetimes with metrics of type ${ }^{F} g_{i j}(x, y)$ and/or ${ }^{F} g_{i j}(x, p)$. For general coordinate transforms, we can omit the left label $F$ and consider structures on certain (co)tangent spaces. Such locally anisotropic metrics can be related to Hessians (8) and/or (6) of respective nonlinear quadratic elements (4) and/or (5) and corresponding N-connection structures. For small values of an indicator of MDRs (1), we suppose that it is possible to preserve the ideas of Universality of Free Fall (and similar Universality of the Gravitational Redshift, or propagation of small perturbations of light/scalar fields etc.) in a FinslerLagrange-Hamilton type generalized spacetime modelled by data (N, g, D) and/or ( 'N, ' $\left.\mathbf{g},{ }^{\prime} \mathbf{D}\right)$. These geometric data can be stated on nonholonomic manifolds with fibred structure and/or on nonholonomic (co)tangent bundles. In such phase spaces (i.e. locally anisotropic spacetime models), the paths of freely falling bodies, and propagation of small perturbations of scalar fields, are not usual geodesics but certain nonlinear (semi-spray) curves which are different from autoparallels of $\mathbf{D}$ and/or 'D. For models of generalized Finsler spacetimes, it is important to study the geometry of semispray configurations (15) as $\mathrm{N}$-connection generalizations of auto-parallel and geodesic curves. In certain sense, semisprays characterize via $\mathrm{N}$-connections and respective adapted nonholonomic variables certain MDRs effects on "physical paths" of test particles.

Working with metric compatible d-connections $\mathbf{D}$ and/or 'D completely determined by some metric and $\mathrm{N}$-connection structures on respective (co)tangent bundles, we can establish a 1-1 correspondence between one type of preferred curves (semi-sprays) and respective auto-parallels. This way, we can encode equivalently the experimental (curvature deviation) 
data with respect to both types of congruences. To derive important physical equations for a Finsler gravitational and matter fields, covariant derivatives determined by a dconnection $\mathbf{D}$, or ' $\mathbf{D}$, must be considered. For canonical constructions, we can work equivalently $\widehat{\mathbf{D}}$, or $\widetilde{\mathbf{D}}$, and respective generalizations for locally anisotropic spinors, see details in Refs. $[147,152,153,159,161,162,167,169,170,179]$. Such a d-connection can be used for defining generalized Dirac, d' Alembert and other physically important operators which allows us to compute the light and particle propagation and study classical and quantum field interactions in a Finsler spacetime and/or Lagrange-Hamilton type modifications.

Principle 3.1 (Modified equivalence principle) In a $M G T$ with an indicator $\varpi\left(x^{i}, E, \overrightarrow{\mathbf{p}}, m ; \ell_{P}\right)(1)$ modeling MDRs and LIVs on (co)tangent bundles, there are nonholonomic variables when point masses, light and small perturbations of scalar fields fall precisely in the same way in an effective phase spacetime along semi-spray configurations (15) and associated auto-parallel equations on $\mathbf{T V}$ or $\mathbf{T}^{*} \mathbf{V}$. Using canonical nonholonomic variables, we can describe such a phase space by $\mathcal{L}$-dual Lagrange and Hamilton geometries (35) with respective canonical d-connections $(\widehat{\mathbf{D}}$ and $\widetilde{\mathbf{D}}$, or ' $\widehat{\mathbf{D}}$ and ' $\widetilde{\mathbf{D}})$. Such canonical and/or almost symplectic $d$-connections are related by canonical distortion relations (34) and can be used for definition of $N$-adapted covariant derivative operators, Dirac operators for locally anisotropic spinors and/or almost symplectic models.

This Principle can be formulated in more general forms for nonholonomic configurations modeling noncommutative and/or supersymmetric MGTs for various types of metric, frame and (non)linear connection structures derived for certain fundamental geometric objects on generalized/analogous manifolds and (co)tangent bundles. Geometrically, such possibilities are motivated by the fact that complex/ noncommutative/supersymmetric configurations can be modelled by certain nonholonomic distributions on real manifolds and/or bundle spaces. In results, we suppose that it can be provided always a self-consistent physical interpretation for theories constructed as nonholonomic deformations of GR and standard particle physics models. Such geometric models can be elaborated for some general data of type $(\mathbf{N}, \mathbf{g}, \mathbf{D})$ which in explicit and non-explicit form involve certain MDRs (1) or in other nonlinear and non-quadratic forms.

\subsubsection{Generalized Mach principles}

The Einstein gravity theory was formulated using a second much less precise set of ideas which goes under the name of Mach's principle. There were involved various philosophical speculations on properties of space and time (in unified forms, spacetime) aether and associated models of continuum mechanics for such an aether media. For elaborating pre-relativity notions of spacetime and classical field theories and in STR, the geometric structure of spacetime is given once and for all and is considered to be unaffected by the material bodies or certain field interactions that may be present. In particular, it was stated that the properties of "inertial motion" and "non-rotating" space are not influenced by matter and fields in the universe.

Mach supposed that all matter in the universe (in a modern fashion, we can include nonlinear effective/observational/ dark matter field interactions and evolution of certain material and fundamental fields configurations) should contribute to the local definition of "non-acceleration" and "nonrotating" of the fundamental space (in a modern approach, spacetime) structure. Einstein accepted this idea and was strongly motivated to formulate a theory where, unlike SRT, the spacetime geometry is influenced by the presence of matter. Such purposes were achieved only partially in GR: the influence of matter was encoded in the right part of the Einstein equations via energy-momentum tensor. In a more general context, we can consider certain effective sources determined cumulatively by generalized connections and/or generic off-diagonal configurations and nonholonomic gravitational distributions and non-minimal coupling to matter fields, for stochastic/kinetic processes, geometric evolution processes etc. Such generalized and nonholonomic spacetime configurations encode more richer geometric vacuum and nonvacuum structures with nontrivial topology, (non)linear symmetries and modified conservation laws. For such off-diagonal gravitational solutions and related spacetime models, the Max principle is extended to a more general set of ideas (still less precise) defining a gravitational spacetime ether with rich structure encoding nonlinear processes (relativistic evolutions and nonlinear interactions) and nonholonomic configurations.

For generalized Finsler gravity theories on (co)tangent bundles derived from classical and/or quantum MDRs, we have to formulate a generalized Mach principle stating that the quantum energy and (non)linear fluctuations, motion of (effective) particles and fields, background metrics and generalized hidden/(non)linear symmetries should all contribute to the spacetime structure modifying it into a nonholonomic phase space. We argue that generalizations to effective phase spaces extending the concept of Lorentz manifolds is motivated by the presence of the quantum world, by kinetic and diffusion processes, by nonlinear off-diagonal interactions and modified symmetries. The influence of such (non)linear effects is encoded both into the nonholonomic structure for data $(\mathbf{N}, \mathbf{g}, \mathbf{D})$ and/or ( ' $\left.\mathbf{N},{ }^{\prime} \mathbf{g},{ }^{\prime} \mathbf{D}\right)$ and into energy-momentum tensors for matter fields embedded selfconsistently in a spacetime aether characterized additionally by velocity, and/or momentum, coordinates $y^{a}$ and/or $p_{a}$. 
Principle 3.2 (Generalized Mach principle) An effective phase space with MDRs on (co) tangent bundles (i.e. a locally anisotropic spacetime modelled as a generalized Finsler-Lagrange-Hamilton geometry) encodes contributions of nonlinear and nonholonomic distortion structures and various types of evolution effects, nonlinear interactions, kinetic and diffusion processes, various nonlinear models with complex structure, nonlinear information, fractional derivative and pattern structure process etc. This consists in a less precise concept of generalized Finsler-Mach principles which can be stated by additional assumptions for explicit models with respective MDRs; certain canonical geometric data and distortions of $(\mathbf{N}, \mathbf{g}, \mathbf{D})$ and/or ( $\left(' \mathbf{N},{ }^{\prime} \mathbf{g},{ }^{\prime} \mathbf{D}\right)$; and effective sources of matter.

Such Finsler-Lagrange-Hamilton generalized Mach principles can be re-formulated, restricted and/or generalized in various forms encoding not only MDRs but also characterizing information processes, complex structures, evolutions of ecological and biophysical systems. The Principle 3.2 can be correlated to more richer geometric structures (commutative and noncommutative ones, algebroids and gerbes, supersymmetric generalizations) in classical and quantum theories, for models of geometric flows, off-diagonal solutions quasi-periodic and/or pattern forming structures etc. Various examples of such constructions determined by exact and parametric solutions were provided and studied in Refs. $[8,9,12,23,69,170,179,191,195,206]$.

\subsubsection{Principle of general covariance and equivalent geometrization of MGTs}

In GR and various classes of MGTs, the principle of general covariance is a natural consequence of geometrization of physical and spacetime models constructed on (pseudo) Riemannian manifolds and metric-affine generalizations. Such theories can be constructed for different Lagrange densities for gravitational and matter fields. This reflects the idea that the geometric and physical constructions should not depend on the type of frames of reference (observers) and coordinate transforms (local parameterizations on some finite, or infinite, regions consisting an atlas covering a spacetime manifold/bundle spaces).

In the definitions of Finsler-Lagrange-Hamilton geometries, the concept of manifold is also involved. For certain classes of MGTs extending the GR to models with MDRs, such manifolds are stated as Lorentz (co)tangent bundle spaces. So, the principles of general covariance has to be extended on some nonholonomic $\mathbf{V}, T \mathbf{V}$ and/or $T^{*} \mathbf{V}$. The N-connection structures for such spaces can be defined in coordinate-free forms encoding MDRs. We can introduce certain "preferred" $\mathrm{N}$-adapted systems of reference and respective coordinate transforms when a fixed $h-v-$ decomposition is prescribed. This does not prohibit us to state respective principles of general covariance for total phase spaces endowed with geometric structures $(\mathbf{N}, \mathbf{g}, \mathbf{D})$ and/or ( 'N, ' $\mathbf{g}$, ' $\mathbf{D})$. For instance, it is possible to define certain nonholonomic canonical variables modeling a pseudo Hamilton space, $(H:$ : ' $\widetilde{\mathbf{g}}, ' \widetilde{\mathbf{N}}, ' \widetilde{\mathbf{D}})$, but such a model can be re-defined by general frame/coordinate transforms and nonholonomic deformations into some general data ( $\mathbf{N}$, ' $\mathbf{g}$, ' $\mathbf{D}$ ), or certain special ones with equivalent almost symplectic variables or allowing a general integration of certain physical important systems of PDEs.

Using not $\mathrm{N}$-adapted frame transforms, all canonical and noncanonical $\mathrm{N}$-adapted constructions can be transformed into certain general frame/coordinate ones, with "hidden" $\mathrm{N}$-connection structures (encoding MDRs) on $V, T V$ and/or $T^{*} V$. In such cases, we can work without boldface symbols and use generic off-diagonal metrics parameterized, respectively, in the forms (20).

The principle of general covariance in GR and MGTs with MDRs can be generalized to a principle of equivalent geometrization of gravitational theories in terms of canonical geometric/physical objects (35) and respective distortion relations (34). Distortions of all geometric and physical objects can be computed following geometric methods (see, for instance, Theorem 2.6). Such distortions of will modify, for instance, the definition of (effective) sources of matter fields and corresponding gravitational an matter filed equations (as we shall prove in next section). Nevertheless, a "dictionary and instructions" for an equivalent geometric formulation of different physical models can be always formulated if the distortions are uniquely determined by the $\mathrm{N}$-connection and metric structures. For instance, performing general nonholonomic frame transforms and deformations, we can geometrize physical theories in general form in terms of geometric objects without "tilde", "hats" etc and work with $\mathrm{N}$-adapted, or not $\mathrm{N}$-adapted, (non)holonomic variables. Such theories can be related to GR and certain classes of MGTs induced on base manifolds via nonholonomic constraints on distortions, when $\mathbf{Z}=0$ and/or ${ }^{\prime} \mathbf{Z}=0$, respectively, for $\mathbf{D}_{\mid \mathbf{Z}=0} \simeq \nabla$ and/or ${ }^{\prime} \mathbf{D}_{\mid} \mid \mathbf{Z}=0$ $\simeq{ }^{\prime} \nabla$, even $\mathbf{D} \neq \nabla$ and/or $\quad \mathbf{D} \neq$ ' $^{\prime} \nabla$. For canonical nonholonomic distortions, we have to impose conditions of type (36) and/or (37).

Principle 3.3 (General covariance and equivalent nonholonomic geometrizations of gravitational theories) $\mathrm{On}$ (co) tangent Lorentz bundles and nonholonomic Lorentz manifolds endowed with $\mathrm{N}$-connection structures (encoding MDRs), it is possible to model geometrically MGTs following principles of general covariance on total and base space manifolds. Using (canonical) distortions such geometric models can be described equivalently for different classes of nonholonomic variables and (nonlinear) connection structures. Imposing 
additional nonholonomic constraints, we can definelextract Levi-Civita configurations with generic off-diagonal metrics.

Above formulated Principles 3.1, 3.2, and 3.3 may involve in a less explicit form various possible multi-connection structures of MGTs with MDRs. In a more general context, considering generic off-diagonal metrics and d-metrics encoding certain background configurations (for instance, like in bi-metric theories, with massive gravitons etc.), we can construct MGTs with multi-metric structures.

\subsubsection{Principles of analogy of $\mathrm{N}$-adapted operators defined by d-metrics and d-connections}

Minimal extensions of GR to (co)tangent Lorentz bundles for models embedding MDRs can be performed by analogy with constructions in pseudo-Riemann geometry (in a more general case, we can use metric-affine and vierbein/spinor geometry) but applying nonholonomic geometric methods and performing respective $\mathrm{N}$-adapted variational, differential, and integral calculi. In abstract forms, there are considered respective Lagrange densities

$$
\begin{aligned}
& \mathcal{L}\left(\mathbf{N}(u), \mathbf{g}(u), \mathbf{D}(u) ;{ }^{\mathbf{A}} \phi(u)\right) \\
& ={ }^{g} \mathcal{L}(\mathbf{N}, \mathbf{g}, \mathbf{D})+{ }^{m} \mathcal{L}\left(\mathbf{N}, \mathbf{g}, \mathbf{D} ;{ }^{A} \phi\right) \text { on } T \mathbf{V} ; \\
& { }_{1} \mathcal{L}\left({ }^{\prime} \mathbf{N}\left({ }^{\prime} u\right),{ }^{\prime} \mathbf{g}\left({ }^{\prime} u\right),{ }^{\prime} \mathbf{D}\left({ }^{\prime} u\right) ;{ }_{1}^{A} \phi\left({ }^{\prime} u\right)\right) \\
& ={ }_{1}^{g} \mathcal{L}\left({ }^{\prime} \mathbf{N},{ }^{\prime} \mathbf{g},{ }^{\prime} \mathbf{D}\right)+{ }_{1}^{m} \mathcal{L}\left({ }^{\prime} \mathbf{N},{ }^{\prime} \mathbf{g},{ }^{\prime} \mathbf{D} ;{ }_{1}{ }_{1} \phi\right) \text { on } T^{*} \mathbf{V} .
\end{aligned}
$$

In these formulas, ${ }^{g} \mathcal{L}$ and ${ }_{i}^{g} \mathcal{L}$ are corresponding (on total spaces of tangent and cotangent bundles) Lagrange densities for modified gravitational fields without matter fields. One can be constructed certain effective matter sources and modeled matter field interactions via distortions of d-connections and/or by introduced bi-/multi-metric structures. Such effective matter terms can be included as additional terms to standard matter Lagrange densities. The respective matter fields ${ }^{A} \phi$ and ${ }_{1}^{A} \phi$ are labeled by an abstract index $A$. Such fields can be scalar, gauge, fermion/spinor fields etc. The explicit constructions of ${ }^{m} \mathcal{L}$ and/or ${ }_{\text {, }}^{m} \mathcal{L}$ depend on the type of theories and phenomenological models we have to elaborate and should be support by a set of experimental and/or observational data which are considered for verifying respective MGTs. In general, (effective) matter Lagrangians are functionals of certain background geometric data $(\mathbf{N}, \mathbf{g}, \mathbf{D})$ and/or ( 'N, ' $\mathbf{g}$, ' D) and encode certain fundamental constants and MDRs, prescribe (non)linear symmetries, assumptions on topological configurations etc. We shall analyze some examples of $\mathcal{L}$ and , $\mathcal{L}$ in Sect. 3.2.

Formulas (39) can be written in canonical variables for respective Finsler/-Lagrange/-Hamilton spaces, i.e. with "tilde" values like ${ }^{m} \widetilde{\mathcal{L}}(\widetilde{\mathbf{N}}, \widetilde{\mathbf{g}}, \widetilde{\mathbf{D}} ; A \widetilde{\phi})$ and necessary type tildes on other Lagrange densities and geometric objects.
Such variables allow and effective Lagrange-Hamilton interpretation of the total phase space/spacetime model, which is convenient for elaborating quantum models. Using general frame and/or coordinate transforms (i.e. Principle 3.3), "tilde" Lagrange densities can be transformed into "hat" Lagrange densities (in such variables, it is possible to decouple and integrate physically important PDEs in general forms). For additional nonholonomic zero distortion conditions, it is possible to extract LC-configurations and project on base Lorentz manifolds in order to find new classes of exact solutions in GR encoding certain information, for instance, about MDRs.

Principle 3.4 (Principle of analogy of Lagrange densities) Gravitational and matter field Lagrange densities considered in GR and MGTs for metric-affine manifolds admit generalizations on (co)tangent Lorentz bundles using nonholonomic variables and deformations of fundamental geometric/physical objects which can be transformed into canonical ones modeling pseudo Finsler/-Lagrange/-Hamilton geometries.

We note that Principles 3.1-3.4 can be formulated in general form for any d-connection $\mathbf{D}$ and/ ' $\mathbf{D}$ (it can be metric compatible or not) related in a unique form via distortion relations to any prescribed LC-connection, canonical d-connection, or almost symplectic connection structures. There are a number of conceptual and technical difficulties in elaborating physical theories with metric noncompatible connections. For instance, there are problems with definition of spinors and Dirac operators, conservation laws, geometric flows etc., see details in Refs. [185, 193,194] and references therein. Nevertheless, self-consistent physical models can be constructed for any well-defined geometrical data generating metric compatible configurations and then distorting in certain unique forms the fundamental physical objects and equations. For more general geometric models and MGTs, we can consider theories with multi-/metric/connection/measure structures. Certain realistic geometric data (35), distortions (34), and Lagrange densities (39) should be fixed in such forms which can be verified by experimental/observational/theoretical simulation data. Such theories for nonholonomic manifolds and tangent bundles (for instance, $\mathbf{T}^{*} \mathbf{V}$ ) were studied in details, see some series of geometric and mathematical physics works with applications in modern cosmology and astrophysics $[9,13,68,70,117,134,144,145,153$, $161,169,175,176,179,181,185,193,194,199-202,207]$. The constructions be can elaborated (generalized, re-defined) on "dual" spaces like $\mathbf{T}^{*} \mathbf{V}$ following certain $\mathcal{L}$-dual, almost symplectic and other type principles. 
3.2 Lagrange densities and energy-momentum tensors on (co)tangent Lorentz bundles

In this subsection, we state the conventions on Lagrange densities and derive respective energy-momentum tensors which will be used for formulating three classes of MGTs with MDRs. We shall consider arbitrary metric compatible d-connections $\mathbf{D}$ and/or ' $\mathbf{D}$.

\subsubsection{Scalar fields on (co)tangent Lorentz bundles}

Let us consider examples of Lagrange densities for matter fields with locally anisotropic interactions:

Convention 3.1 (Scalar fields with MDRs on (co)tangent bundles) Scalar field interactions on phase spaces with MDRs can be modeled by Lagrange densities

$$
\begin{aligned}
& { }^{m} \mathcal{L}={ }^{\phi} \mathcal{L}(\mathbf{g} ; \phi) \text { on } T \mathbf{V} \text { and/or } \\
& { }_{\text {। }}^{m} \mathcal{L}={ }_{\text {। }}^{\phi} \mathcal{L}\left({ }^{\prime} \mathbf{g} ; \text {, } \phi\right) \text { on } T^{*} \mathbf{V}
\end{aligned}
$$

depending only on d-metrics, $\mathbf{g}_{\mu \nu}$ and/or ${ }^{\prime} \mathbf{g}^{\alpha \beta}$ (this allows to construct exact off-diagonal solutions in explicit form, see discussion and references for Direction 10 in Appendix $B$ of [208], and on scalar fields ${ }^{A} \phi=\phi(u)$ and ${ }_{1}^{A} \phi={ }_{1} \phi(' u)$.

Performing a $\mathrm{N}$-adapted variational calculus, we prove

Consequence 3.1 (Energy-momentum d-tensors for locally anisotropic interacting scalar fields) The symmetric energymomentum d-tensors for scalar fields on (co)tangent bundles derived for respective Lagrange densities (40) are computed for possible $h$ - and v-, or cv-splitting

$$
\begin{aligned}
& { }^{\phi} \mathbf{T}_{\alpha \beta}=-\frac{2}{\sqrt{\left|\mathbf{g}_{\mu \nu}\right|}} \frac{\delta\left(\sqrt{\left|\mathbf{g}_{\mu \nu}\right|}{ }^{\phi} \mathcal{L}\right)}{\delta \mathbf{g}^{\alpha \beta}}={ }^{\phi} \mathcal{L} \mathbf{g}_{\alpha \beta}+2 \frac{\delta\left({ }^{\phi} \mathcal{L}\right)}{\delta \mathbf{g}^{\alpha \beta}} \\
& =\left\{{ }^{\phi} \mathbf{T}_{i j}=-\frac{2}{\sqrt{\left|\mathbf{g}_{\mu \nu}\right|}} \frac{\delta\left(\sqrt{\left|\mathbf{g}_{\mu \nu}\right|}{ }^{\phi} \mathcal{L}\right)}{\delta \mathbf{g}^{i j}}\right. \\
& \left.=\ldots,{ }^{\phi} \mathbf{T}_{a b}=-\frac{2}{\sqrt{\left|\mathbf{g}_{\mu \nu}\right|}} \frac{\delta\left(\sqrt{\left|\mathbf{g}_{\mu \nu}\right|}{ }^{\phi} \mathcal{L}\right)}{\delta \mathbf{g}^{a b}}=\ldots\right\} ; \\
& { }_{1}^{\phi} \mathbf{T}_{\alpha \beta}=-\frac{2}{\sqrt{\left|{ }^{\prime} \mathbf{g}_{\mu \nu}\right|}} \frac{\delta\left(\sqrt{\left|{ }^{\prime} \mathbf{g}_{\mu \nu}\right|}{ }_{1}^{\phi} \mathcal{L}\right)}{\delta^{\prime} \mathbf{g}^{\alpha \beta}} \\
& ={ }_{1}^{\phi} \mathcal{L}{ }^{\prime} \mathbf{g}_{\alpha \beta}+2 \frac{\delta\left(\begin{array}{c}
\phi \\
\text { । }
\end{array}\right)}{\delta^{\prime} \mathbf{g}^{\alpha \beta}}
\end{aligned}
$$

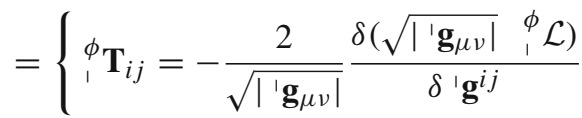

$$
\begin{aligned}
& =\ldots,{ }_{1}^{\phi} \mathbf{T}^{a b}=-\frac{2}{\sqrt{\left|{ }^{\prime} \mathbf{g}_{\mu \nu}\right|}} \frac{\delta\left(\sqrt{\left|{ }^{\prime} \mathbf{g}_{\mu \nu}\right|}{ }_{i}^{\phi} \mathcal{L}\right)}{\delta^{\prime} \mathbf{g}_{a b}} \\
& =\ldots\} \text {. }
\end{aligned}
$$

Scalar field equations can be re-defined by an additional $3+1$ splitting on base and fiber spaces as certain moving equations for ideal fluids. For instance, we can consider a velocity $\mathbf{v}_{\alpha}$ d-vector subjected to the conditions $\mathbf{v}_{\alpha} \mathbf{v}^{\alpha}=1$ and $\mathbf{v}^{\alpha} \mathbf{D}_{\beta} \mathbf{v}_{\alpha}=0$, for ${ }^{\phi} \mathcal{L}:=-p$ in a corresponding local $\mathrm{N}$-adapted frame.

Remark 3.1 (Energy-momentum d-tensors for locally anisotropic perfect liquids) Conventionally, the sources (41) can be approximated as a perfect liquid matter (we use a left abstract index "l" for liquid) with respective density and pressure

$$
\begin{aligned}
& { }^{l} \mathbf{T}_{\alpha \beta}=(\rho+p) \mathbf{v}_{\alpha} \mathbf{v}_{\beta}-p \mathbf{g}_{\alpha \beta} \text { and/or } \\
& { }_{1}^{l} \mathbf{T}_{\alpha \beta}=\left({ }^{\prime} \rho+{ }^{\prime} p\right){ }^{\prime} \mathbf{v}_{\alpha}{ }^{\prime} \mathbf{v}_{\beta}-{ }^{\prime} p{ }^{\prime} \mathbf{g}_{\alpha \beta} .
\end{aligned}
$$

It should be noted that formulas (41) do not depend in explicit form on d-connections. Nevertheless, such dependencies should be considered if $\phi(u)$ and/or $\phi($ ' $u)$ are subjected to the conditions to be solutions of some generalized scalar field equations (Klein-Gordon, or other types, for instance, certain hydrodynamical equations). Using respective nonholonomic variables, we can re-write these formulas in terms of "tilde/hat" variables.

\subsubsection{Lagrange densities for Einstein-Yang-Mills-Higgs systems with MDRs}

Various models of locally anisotropic gravitational and gauge field interactions on modified Finsler spaces, nonholonomic Lorentz manifolds, and higher order Lagrange-Hamilton spaces were studied in a series of our works [10,18,23,142$146,153,161,169,173,175,190,203,209]$. That research was on twistor and spinor methods for generalized geometries, with nearly autoparallel maps and generalized connections, for formulating models of Finsler gravity as gauge like theories, for supersymmetric generalizations and, more recently, superstring and supergravity theories. Those directions were related also to the theory of spinors in Finsler-LagrangeHamilton spaces and modelling such configurations, for instance, in brane gravity, with Clifford-algebroids, noncommutative Finsler geometry and locally anisotropic Dirac operators [147,159,162,179]. We shall present a detailed study of Einstein-Dirac systems with MDRs resulting in Clifford-Lagrange-Hamilton geometries, and constructed exact solutions and quantum models, in other partner works (see also Directions 3 and 7, respectively, in Appendix B of [208]. In this subsection, we define Lagrange densities and corresponding energy-momentum tensors for certain models of Yang-Mills-Higgs, YMH, interactions on (co)tangent Lorentz bundles.

Let us consider YMH systems modeled on nonholonomic Lorentz manifolds, or on their (co)tangent bundles, by respective geometric data $\left(\mathbf{N}, \mathbf{g}, \mathbf{D} ; \mathbf{A}^{\check{a}}(u), \phi^{\check{a}}(u)\right)$ and/or 
( 'No, 'g, ' $\mathbf{D}$; ' $\mathbf{A}^{\check{a}}(u)$, ' $\left.\phi^{\check{a}}\left({ }^{\prime} u\right)\right)$ for matter fields parameterized in the form ${ }^{A} \phi=\left[\mathbf{A}^{\breve{a}}(u), \phi^{\breve{a}}(u)\right]$ and/or ${ }_{1}^{A} \phi=$ $\left[\_A^{\check{a}}\left({ }^{\prime} u\right),{ }_{1} \phi^{\check{a}}\left({ }^{\prime} u\right)\right]$. In these formulas, the non-Abelian gauge fields $\mathbf{A}=\left\{\mathbf{A}^{\check{a}}(u)\right\}$ can be defined by a 1 -form $\mathbf{A}=\mathbf{A}_{\delta} d u^{\delta}$ when coefficients $\mathbf{A}_{\delta}^{\check{a}}$ take values in a Lie algebra $\mathcal{A}$ of so-called internal gauge symmetries. The index $\check{a}$ labels elements of such Lie algebra and related groups. For $\mathcal{A}=s u(2)$ and base Minkowski spaces, we obtain standard Yang-Mills fields. The scalar-multiplet (complex) field $\phi^{\check{a}}$ is called the Higgs field. The model is elaborated on (co)tangent bundles with respective 1 -forms

$$
\begin{aligned}
& \mathbf{A}=\mathbf{A}_{\delta} d u^{\delta}, \quad \text { for } \mathbf{A}_{\delta}=\mathbf{A}_{\delta}^{\check{a}} \tau_{\breve{a}}^{\check{a}}, \text { and } \\
& { }^{\prime} \mathbf{A}={ }^{\prime} \mathbf{A}_{\delta} d u^{\delta}, \quad \text { for }{ }^{\prime} \mathbf{A}_{\delta}={ }^{\prime} \mathbf{A}_{\delta}^{\check{a}} \tau_{\breve{a}},
\end{aligned}
$$

where $\tau_{\check{a}}$ are generators of $\mathcal{A}$. We consider summation on repeating indices $\check{a}$ in all cases "up-low", "up-up", and "lowlow". In this work, we consider gauge theories with the same gauge group both for tangent and cotangent bundles, extended to respective vector bundles with typical fibers and respective adjoint representations of Lie algebra of $\mathcal{A}$.

Convention 3.2 (Covariant derivatives of gauge fields in (co)tangent bundles) The non-Abelian gauge fields (on (co)tangent bundles enabled with respective metric compatible d-connections $\mathbf{D}$ and $\mathbf{D})$ define covariant derivatives in the associated vector bundles,

$\mathcal{D}_{\delta}=\mathbf{D}_{\delta}+i \check{e}\left[\mathbf{A}_{\delta}, \cdot\right]$ and/or ${ }^{\prime} \mathcal{D}_{\delta}={ }^{\prime} \mathbf{D}_{\delta}+i \check{e}\left[{ }^{\prime} \mathbf{A}_{\delta}, \cdot\right]$,

where $\check{e}$ is the coupling constant, $i^{2}=-1$, and $[\cdot, \cdot]$ is used for the commutator.

We note that such values should be written with "hat" or "tilde" symbols if the phase space d-connections are of respective type, for instance, $\widehat{\mathcal{D}}_{\delta}=\widehat{\mathbf{D}}_{\delta}+i \check{e}\left[\widehat{\mathbf{A}}_{\delta}, \cdot\right]$. All formulas derived with "hat/tilde" operators are labeled by "hat/tilde" symbols.

By a standard differential form calculus, we prove

Definition 3.1 -Theorem (Strengths of gauge fields in curved phase spaces) The d-vector fields $\mathbf{A}_{\delta}$ and ' $\mathbf{A}_{\mu}$ are characterised by respective curvatures

$$
\begin{aligned}
\mathcal{F}_{\beta \mu} & :=\mathbf{D}_{\beta} \mathbf{A}_{\mu}-\mathbf{D}_{\mu} \mathbf{A}_{\beta}+i \check{e}\left[\mathbf{A}_{\beta}, \mathbf{A}_{\mu}\right] ; \\
{ }^{\prime} \mathcal{F}_{\beta \mu} & :={ }^{\prime} \mathbf{D}_{\beta}{ }^{\prime} \mathbf{A}_{\mu}-{ }^{\prime} \mathbf{D}_{\mu} \mathbf{A}_{\beta}+i \check{e}\left[{ }^{\prime} \mathbf{A}_{\beta},{ }^{\prime} \mathbf{A}_{\mu}\right] .
\end{aligned}
$$

Following Principle 3.4, we formulate:

Convention 3.3 (Lagrange densities for YMH interactions on (co)tangent Lorentz bundles) Non-Abelian gauge-Higgs, $Y M H$, interactions on phase spaces with MDRs can be modeled by Lagrange densities

$$
\begin{aligned}
& { }^{A} \mathcal{L}=-\frac{1}{4} \mathcal{F}^{\check{a} \mu \nu} \mathcal{F}_{\mu \nu}^{\check{a}} \quad \text { and } \\
& { }^{H} \mathcal{L}=-\frac{1}{2}\left(\mathcal{D}_{\mu} \phi^{\check{a}}\right)\left(\mathcal{D}^{\mu} \phi^{\check{a}}\right)-\mathcal{V}\left(\phi^{\check{a}}\right) ;
\end{aligned}
$$

$$
\begin{aligned}
& { }_{1}^{A} \mathcal{L}=-\frac{1}{4}{ }^{\prime} \mathcal{F}^{\check{a} \mu \nu}{ }^{\prime} \mathcal{F}_{\mu \nu}^{\check{a}} \text { and } \\
& { }_{1}^{H} \mathcal{L}=-\frac{1}{2}\left({ }^{\prime} \mathcal{D}_{\mu}{ }^{\prime} \phi^{\check{a}}\right)\left({ }^{\prime} \mathcal{D}^{\mu}{ }^{\prime} \phi^{\check{a}}\right)-{ }^{\prime} \mathcal{V}\left({ }^{\prime} \phi^{\check{a}}\right),
\end{aligned}
$$

were $\mathcal{V}\left(\phi^{\check{a}}\right)=\frac{1}{4} \check{\lambda}\left(\left|\phi_{[0]}^{\check{a}}\right|^{2}-\bar{\phi}^{\check{a}} \phi^{\check{a}}\right)^{2}$ and $\mathcal{V}\left({ }^{\prime} \phi^{\check{a}}\right)=$

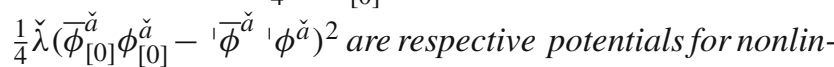
ear interactions of Higgs fields with corresponding vacuum expectations $\left|\phi_{[0]}^{\check{a}}\right|^{2}=\bar{\phi}_{[0]}^{\check{a}} \phi_{[0]}^{\check{a}}$ and $\left|{ }^{\prime} \phi_{[0]}^{\check{a}}\right|^{2}={ }^{\prime} \bar{\phi}_{[0]}^{\check{a}}{ }^{\prime} \phi_{[0]}^{\check{a}}$ and self-integration constant $\check{\lambda}$.

Above considered constants can be related to physical ones, for instance, in the Weinberg-Salam theory when vacuum expectation of the Higgs field which determines a masse ${ }^{H} M=\sqrt{\check{\lambda}} \eta$; the mass of W-bosons is ${ }^{W} M=e v$.

Performing a $\mathrm{N}$-adapted variational calculus, we prove

Consequence 3.2 (Energy-momentum d-tensors for YMH fields with locally anisotropic interactions) The symmetric energy-momentum d-tensors for scalar fields on (co)tangent bundles derived for respective Lagrange densities (44) are computed for locally anisotropic gauge fields,

$$
\begin{aligned}
& { }^{A} \mathbf{T}_{\beta \delta}=2\left(\mathbf{g}^{\mu \nu} \mathcal{F}_{\beta \mu}^{\check{a}} \mathcal{F}_{\nu \delta}^{\check{a}}-\frac{1}{4} \mathbf{g}_{\beta \delta} \mathcal{F}^{\check{a} \mu \nu} \mathcal{F}_{\mu \nu}^{\check{a}}\right) \quad \text { and } \\
& { }_{1}^{A} \mathbf{T}_{\beta \delta}=2\left(\mathbf{g}^{\mu \nu}{ }^{\prime} \mathcal{F}_{\beta \mu}^{\check{a}}{ }^{\prime} \mathcal{F}_{\nu \delta}^{\check{a}}-\frac{1}{4}{ }^{\prime} \mathbf{g}_{\beta \delta}{ }^{\prime} \mathcal{F}^{\check{a} \mu \nu}{ }^{\prime} \mathcal{F}_{\mu \nu}^{\check{a}}\right),
\end{aligned}
$$

and for Higgs fields,

$$
\begin{aligned}
{ }^{H} \mathbf{T}_{\beta \delta}= & \frac{1}{4}\left(\mathcal{D}_{\delta} \bar{\phi}^{\check{a}} \mathcal{D}_{\beta} \phi^{\check{a}}+\mathcal{D}_{\beta} \bar{\phi}^{\check{a}} \mathcal{D}_{\delta} \phi^{\check{a}}\right) \\
& -\frac{1}{4} \mathbf{g}_{\beta \delta} \mathcal{D}_{\alpha} \bar{\phi}^{\check{a}} \mathcal{D}^{\alpha} \phi^{\check{a}}-\mathbf{g}_{\beta \delta} \mathcal{V}\left(\phi^{\check{a}}\right) ; \\
{ }_{1}^{H} \mathbf{T}_{\beta \delta}= & \frac{1}{4}\left({ }^{\prime} \mathcal{D}_{\delta}{ }^{\prime} \bar{\phi}^{\check{a}}{ }^{\prime} \mathcal{D}_{\beta}{ }^{\prime} \phi^{\check{a}}+{ }^{\prime} \mathcal{D}_{\beta}{ }^{\prime} \bar{\phi}^{\check{a}}{ }^{\prime} \mathcal{D}_{\delta}{ }^{\prime} \phi^{\check{a}}\right) \\
& -\frac{1}{4}{ }^{\prime} \mathbf{g}_{\beta \delta}{ }^{\prime} \mathcal{D}_{\alpha}{ }^{\prime} \bar{\phi}^{\check{a}}{ }^{\prime} \mathcal{D}^{\alpha}{ }^{\prime} \phi^{\check{a}}-{ }^{\prime} \mathbf{g}_{\beta \delta}{ }^{\prime} \mathcal{V}\left({ }^{\prime} \phi^{\check{a}}\right) .
\end{aligned}
$$

Similar formulas can be derived for models of quantum chromodynamics, QCD, with MDRs generalized on (co)tangent Lorentz bundles. In this work, we omit geometric constructions with the gauge Lie algebra $\mathcal{A}=s u(3)$ resulting in locally anisotropic interactions of gluonic and quark fields on (co)tangent Lorentz bundles.

\subsubsection{Actions for minimal MDR-extensions of GR and YMH theories}

In GR, the Lagrange density for gravitational fields is postulated in the form

$$
{ }^{g} \mathcal{L}\left(g_{i j}, \nabla\right)=\frac{{ }^{P l} M^{2}}{2} R,
$$

where $R$ is the Ricci scalar of a Lorentz manifold $V$ and the Planck mass ${ }^{P l} M$ is determined by the Newton constant ${ }^{N e w} G$. In the units ${ }^{N e w} G=1 / 16 \pi$ with ${ }^{P l} M=$ 
$\left(8 \pi^{\text {New }} G\right)^{-1 / 2}=\sqrt{2}$, one states a constant $\varkappa$ for the matter source $\Upsilon_{i j}:=\varkappa\left(T_{i j}-\frac{1}{2} g_{i j} T\right)$, where $T:=g^{i j} T_{i j}$, for $T_{k l}:=-\frac{2}{\sqrt{\left|\mathbf{g}_{i j}\right|}} \frac{\delta\left({\sqrt{\left|\mathbf{g}_{i j}\right|}}^{m} \mathcal{L}\right)}{\delta \mathbf{g}^{k l}}$ with Lagrange density of matter fields ${ }^{m} \mathcal{L}$. The Einstein equations with the Ricci tensor for $\nabla$,

$R_{i j}=\Upsilon_{i j}$,

can be derived by a variational calculus on $V$ using action $\mathcal{S}={ }^{g} \mathcal{S}+{ }^{m} \mathcal{S}=\frac{1}{16 \pi} \int d^{4} x \sqrt{\left|g_{i j}\right|}\left({ }^{g} \mathcal{L}+{ }^{m} \mathcal{L}\right)$.

Let us consider on $T \mathbf{V}$ a metric compatible d-connection $\mathbf{D}$ completely defined by a d-metric $\mathbf{g}$ and with the property that for respective nonholonomic constraints $\mathbf{D}_{\mid \mathcal{T}=0}=\nabla$ (such constraints may be non-integrable and or may not have a smooth limit). Following Principle 3.4, we consider that minimal extensions of GR to tangent Lorentz bundles enabled with data (N, g, D) should be determined by nonholonomically deformed Einstein equations of type

$\mathbf{R}_{\alpha \beta}[\mathbf{D}]=\Upsilon_{\beta \gamma}:=\varkappa\left(\mathbf{T}_{\alpha \beta}-\frac{1}{2} \mathbf{g}_{\alpha \beta} T\right)$,

where $T:=\mathbf{g}^{\alpha \beta} \mathbf{T}_{\alpha \beta}$ and the constant $\varkappa$ may take different values on $\mathrm{h}$ - and $\mathrm{v}$-subspaces (this should be defined by experimental/observational data). Similar geometric formulas can be written on $T^{*} \mathbf{V}$, for instance, as ${ }^{\prime} \mathbf{R}_{\alpha \beta}\left[{ }^{\prime} \mathbf{D}\right]={ }^{\prime} \Upsilon_{\beta \gamma}$ (when splitting into $h$ - and $c v$-components can be considered with, or not, $L$-duality and with additional requests on possible almost symplectic symmetries, see below necessary details).

Summarizing Lagrange densities introduced in previous subsections, we formulate:

Principle 3.5 -Convention (Actions for minimally MDRmodified EYMH systems) Locally anisotropic interactions of Einstein gauge and Higgs fields on (co)tangent Lorentz bundles (endowed with metric compatible d-connections uniquely determined by respective d-metric structures and admitting nonholonomic variables for distinguishing FinslerLagrange-Hamilton phase spaces) are described by respective actions

$$
\begin{aligned}
\mathcal{S} & ={ }^{\mathbf{g}} \mathcal{S}+{ }^{\phi} \mathcal{S}+{ }^{A} \mathcal{S}+{ }^{H} \mathcal{S} \\
& =\frac{1}{16 \pi} \int \delta u \sqrt{\left|\mathbf{g}_{\alpha \beta}\right|}\left({ }^{\mathbf{g}} \mathcal{L}+{ }^{\phi} \mathcal{L}+{ }^{A} \mathcal{L}+{ }^{H} \mathcal{L}\right) ; \\
\mathcal{S} & ={ }^{\mathbf{g}} \mathcal{S}+{ }_{1} \mathcal{S}+{ }_{1}^{A} \mathcal{S}+{ }^{H} \mathcal{S} \\
& =\frac{1}{16 \pi} \int \delta{ }^{\prime} u \sqrt{\left|\mathbf{I}_{\alpha \beta}\right|}\left({ }_{1}{ }^{\mathbf{g}} \mathcal{L}+{ }_{1}^{\phi} \mathcal{L}+{ }_{1}^{A} \mathcal{L}+{ }_{1}^{H} \mathcal{L}\right),
\end{aligned}
$$

where ${ }^{\mathbf{g}} \mathcal{L}:={ }^{s} \mathbf{R}=\mathbf{g}^{\alpha \beta} \mathbf{R}_{\alpha \beta}[\mathbf{D}],{ }_{1}^{\mathbf{g}} \mathcal{L}:={ }_{1}^{s} \mathbf{R}=$ ${ }^{\prime} \mathbf{g}^{\alpha \beta}{ }^{\prime} \mathbf{R}_{\alpha \beta}$ [' $\left.\mathbf{D}\right]$ and the Lagrange densities for scalar-YMH fields are given by corresponding formulas (40) and (44).

Performing a $\mathrm{N}$-adapted variational calculus and summarizing previous Consequences on energy-momentum dtensors, we prove
Consequence 3.3 (Sources for locally anisotropic YMH fields) On (co)tangent Lorentz bundles, minimal modifications of scalar and YMH systems encoding MDRs are characterized by respective sources

$$
\begin{aligned}
& { }^{\phi} \Upsilon_{\beta \gamma}:=\varkappa\left({ }^{\phi} \mathbf{T}_{\alpha \beta}-\frac{1}{2} \mathbf{g}_{\alpha \beta}{ }^{\phi} T\right), \\
& { }^{A} \Upsilon_{\beta \gamma}:=\varkappa\left({ }^{A} \mathbf{T}_{\alpha \beta}-\frac{1}{2} \mathbf{g}_{\alpha \beta}{ }^{A} T\right), \\
& { }^{H} \Upsilon_{\beta \gamma}:=\varkappa\left({ }^{H} \mathbf{T}_{\alpha \beta}-\frac{1}{2} \mathbf{g}_{\alpha \beta}{ }^{H} T\right) ; \\
& { }^{\phi} \Upsilon_{\beta \gamma}:=\varkappa\left({ }^{\phi} \mathbf{T}_{\alpha \beta}-\frac{1}{2}{ }^{1} \mathbf{g}_{\alpha \beta}{ }_{1} T\right), \\
& { }^{A} \Upsilon_{\beta \gamma}:=\varkappa\left({ }^{A}{ }^{{ }^{A}} \mathbf{T}_{\alpha \beta}-\frac{1}{2}{ }^{\prime} \mathbf{g}_{\alpha \beta}{ }_{1}{ }^{A} T\right), \\
& { }^{H} \Upsilon_{\beta \gamma}:=\varkappa\left({ }^{H} \mathbf{T}_{\alpha \beta}-\frac{1}{2}{ }^{{ }^{H}} \mathbf{g}_{\alpha \beta}{ }_{1}{ }^{H} T\right),
\end{aligned}
$$

where the energy-momentum tensors are given respectively by formulas (41), (45) and (46).

In GR, the sources of matter fields are approximated (for instance, for quantum fluctuations or certain summarized contributions) to (effective) cosmological constants. Following Principle 3.4, we consider

Assumption 3.1 (Effective cosmological constants for locally anisotropic YMH sources) Generalized sources for matter fields (including possible effective sources defined by distortion tensors of d-connections) can be approximated by respective cosmological constants $\Lambda$ and/or,$\Lambda$,

$$
\Upsilon_{\gamma}^{\beta}=\Lambda \delta_{\gamma}^{\beta}{ }_{\gamma} \text { and/or }{ }_{1} \Upsilon_{\gamma}^{\beta}{ }_{\gamma}={ }_{,} \Lambda \delta^{\beta}{ }_{\gamma} \text {, }
$$

when, correspondingly, $\Upsilon_{\beta \gamma}={ }^{\phi} \Upsilon_{\beta \gamma}+{ }^{A} \Upsilon_{\beta \gamma}+{ }^{H} \Upsilon_{\beta \gamma}$ and/or ${ }_{1} \Upsilon_{\beta \gamma}={ }_{1}^{\phi} \Upsilon_{\beta \gamma}+{ }_{1}^{A} \Upsilon_{\beta \gamma}+{ }_{1}^{H} \Upsilon_{\beta \gamma}$, considering that such sources are subjected to relations of additivity,

$$
\begin{aligned}
& \Lambda={ }^{\phi} \Lambda+{ }^{A} \Lambda+{ }^{H} \Lambda \text { and/or } \\
& { }_{1} \Lambda={ }_{1}^{\phi} \Lambda+{ }_{1}^{A} \Lambda+{ }_{1}^{H} \Lambda .
\end{aligned}
$$

We note that all such cosmological constants can be zero, positive, or negative. They may compensate each other and result in (fictive) vacuum configurations, or take different values in certain $h-, v$-, and/or $c v$-subspaces.

Remark 3.2 (Effective cosmological constants as nonlinear superpositions of associated cosmological constants for locally anisotropic YMH fields) In certain MGTs with quasiclassical corrections, renormalizations, nonlinear MDRs, nonlinear symmetries of generating functions and/or (effective) sources, nonlinear vacuum polarizations etc., one can be considered nonlinear functionals of type $\Lambda=$ $\Lambda\left[{ }^{\phi} \Lambda,{ }^{A} \Lambda,{ }^{H} \Lambda\right]$ and/or ${ }_{1} \Lambda={ }_{1} \Lambda\left[{ }_{1}^{\phi} \Lambda,{ }_{1}^{A} \Lambda,{ }_{1}^{H} \Lambda\right]$. We do not study such theories in this work. 


\subsubsection{Actions for MGTs with massive gravitons, bi-metric structures and MDRs}

A large class of MGTs are constructed with modifications of Lagrange density (47) to a general functional depending on Ricci scalar, torsion, energy-momentum and other fundamental geometric/physical values. For instance, there are considered modifications of type ${ }^{g} \mathcal{L}\left(g_{i j}, \nabla\right) \approx R \rightarrow$ $f(R)$, where $R$ is a scalar curvature determined by the LCconnection or a generalized metric-affine connection. During last 20 years, it was elaborated a paradigm of $f$-modified gravity theories attempting to explain the universe acceleration and solve the dark energy and dark matter problems. In a more general context, this paradigm involves theories with infra-red (IR) modifications of the GR theory and ultra-violet (UV) corrections expected to be of quantum origin. Various studies were on understanding possible physical implications of the massive spin-2 theory, MDRs and LIVs, generalized Finsler gravity theories, commutative and noncommutative/nonassociative and/or supersymmetric generalizations etc. A series of recent our works [68,69,198-203,207] is devoted to elaborating nonholonomic $f$-modified theories and study of cosmological implications of massive gravity and bi-metric gravity with local anisotropy. Such constructions were inspired by papers on holonomic models of nonlinear massive gravitational theories including $f(R)$ modifications $[24,76,106]$. Here we emphasize that locally (an)isotropic massive gravity theories contain the benefits of the so-called dRGT model and generalizations, see $[113,114,135,210]$, being free of ghost modes $[22,47]$. Advantages are those that by tuning the $f(R)$ and various MDR-deformed functionals (for holonomic configurations, see reviews $[26,105])$, we can relate locally anisotropic black hole solutions, stabilize cosmological backgrounds, elaborate various types of locally anisotropic cosmological evolution scenarios and provide for MGTs an unified description of inflation and late-time acceleration etc. In this paper, we geometrize specific models of massive $f(R)$ gravity with MDRs and (see subsections below) the corresponding systems of modified gravitational equations.

We extend the approaches elaborated in $[68,69,200$ $203,207]$ and $[24,76,106]$ and consider theories on nonholonomic (co)tangent Lorentz bundles enabled with a common $\mathrm{N}$-connection structure $\mathbf{N}$ for two d-metrics where $\mathbf{g}=\left\{\mathbf{g}_{\alpha \beta}\right\}$ is a dynamical d-metric and $\mathbf{q}=\left\{\mathbf{q}_{\alpha \beta}\right\}$ is a non-dynamical reference metric. For Lagrange theories, we work with a metric compatible d-connection $\mathbf{D}$ (instead of the LC-connection $\nabla$ ) and a corresponding Ricci scalar $s R$ both computed for g. On a base Lorentz manifold, the nonzero graviton mass is denoted by $\mu$, the constant $M_{P}$ is the Planck mass and such constants are lifted with re-definition on fiber space (on total phase spaces, such values have to be defined experimentally).
For elaborating Hamilton massive and bi-metric gravity theories, there are considered corresponding values: a $\mathrm{N}$ connection structure, $\mathbf{N}$ for, $\mathbf{g}=\left\{{ }_{,} \mathbf{g}_{\alpha \beta}\right\}$ being the dynamical d-metric and ${ }_{,} \mathbf{q}=\left\{{ }_{,} \mathbf{q}_{\alpha \beta}\right\}$ being the so-called non-dynamical reference (fiducial) d-metric, when respective ${ }_{1} \mathbf{D}$ and ${ }_{s} R$ are computed for $\mathbf{g}$. Let us consider the d-tensor

$$
\begin{aligned}
\left(\sqrt{\mathbf{g}^{-1} \mathbf{q}}\right)_{v}^{\mu} & =\left(\left(\sqrt{g^{-1} q}\right)_{j}^{i},\left(\sqrt{g^{-1} q}\right)_{b}^{a}\right), \text { and/or } \\
\left(\sqrt{\mathbf{g}^{-1}, \mathbf{q}}\right)_{v}^{\mu} & =\left(\left(\sqrt{g^{-1}{ }^{-1} q}\right)_{j}^{i},\left(\sqrt{{ } g^{-1}, q}\right)_{a}^{b}\right), \\
\text { i.e. }\left(\sqrt{\mathbf{g}^{-1}, \mathbf{q}}\right) & =\left(\sqrt{h\left(, g^{-1}, q\right)}, \sqrt{v\left(, g^{-1}, q\right)}\right), \text { and/or } \\
\left(\sqrt{\mathbf{g}^{-1}, \mathbf{q}}\right) & =\left(\sqrt{h\left(, g^{-1}, q\right)}, \sqrt{c v\left(, g^{-1}, q\right)}\right),
\end{aligned}
$$

where the square roots are computed respectively for $\mathbf{g}^{\mu \rho} \mathbf{q}_{\rho v}$ and/or ${ }_{i} \mathbf{g}^{\mu \rho}{ }_{1} \mathbf{q}_{\rho \nu}$, when

$$
\begin{aligned}
& \left(\sqrt{g^{-1} q}\right)_{j}^{i}\left(\sqrt{g^{-1} q}\right)_{k}^{j}=g^{i j} q_{j k}, \quad \text { and } \\
& \sum_{k=0}^{4}{ }_{h}^{k} \beta_{h} e_{k}\left(\sqrt{h\left(g^{-1} q\right)}\right)=3-\operatorname{tr} \sqrt{h\left(g^{-1} q\right)} \\
& \quad-\operatorname{det} \sqrt{h\left(g^{-1} q\right)} ; \\
& \left(\sqrt{g^{-1} q}\right)_{b}^{a}\left(\sqrt{g^{-1} q}\right)_{c}^{b}=g^{a b} q_{b c}, \quad \text { and } \\
& \sum_{k=0}^{4}{ }_{v}^{k} \beta_{v} e_{k}\left(\sqrt{v\left(g^{-1} q\right)}\right)=3-\operatorname{tr} \sqrt{v\left(g^{-1} q\right)} \\
& -\operatorname{det} \sqrt{v\left(g^{-1} q\right)} ;
\end{aligned}
$$

and/or $\left(\sqrt{{ }_{1} g^{-1}, q}\right)_{j}^{i}\left(\sqrt{{ } g^{-1}, q}\right)_{k}^{j}={ }_{1} g^{i j}{ }_{1} q_{j k}$, and

$$
\begin{aligned}
& \sum_{k=0}^{4}{\stackrel{o}{h}{ }_{h}}_{\beta}{ }_{h} e_{k}\left(\sqrt{h\left({ }_{1} g^{-1}, q\right)}\right)=3-\operatorname{tr} \sqrt{h\left({ }_{1} g^{-1}, q\right)} \\
& -\operatorname{det} \sqrt{h\left({ }_{1} g^{-1}, q\right)} \text {; } \\
& \left(\sqrt{{ }_{1} g^{-1}{ }_{1} q}\right)_{a}^{b}\left(\sqrt{{ }_{1} g^{-1}, q}\right)_{b}^{c}={ }_{1} g_{a b}, q^{b c}, \text { and } \\
& \sum_{k=0}^{4}{ }_{k v 1}^{k} \beta_{c v 1} e_{k}\left(\sqrt{c v\left(, g^{-1}, q\right)}\right)=3-\operatorname{tr} \sqrt{c v\left(, g^{-1}, q\right)} \\
& -\operatorname{det} \sqrt{c v\left(, g^{-1}, q\right)}
\end{aligned}
$$

for respective coefficients ${ }_{h}^{k} \beta,{ }_{v}^{k} \beta,{ }_{h 1}^{\circ} \beta$ and ${ }_{c v 1}^{\circ} \beta$. The values ${ }_{h} e_{k},{ }_{v} e_{k}$ and/or ${ }_{h} e_{k},{ }_{c v} e_{k}$ can be computed correspondingly for any d-tensor $\mathbf{X}_{\rho}^{\mu}=\left(X^{i}{ }_{j}, X_{b}^{a}\right)$ with trace 


$$
\begin{aligned}
X & ={ }^{h} X+{ }^{v} X=\left[{ }^{h} X\right]+\left[{ }^{v} X\right]:=\operatorname{tr}(\mathbf{X}) \\
& =\operatorname{tr}\left({ }^{h} X\right)+\operatorname{tr}\left({ }^{v} X\right)=\mathbf{X}^{\mu}{ }_{\mu}=X_{i}^{i}+X^{a}{ }_{a}
\end{aligned}
$$

and/or any d-tensor ${ }_{1} \mathbf{X}_{\rho}^{\mu}=\left({ }_{1} X^{i}{ }_{j},{ }_{1} X_{a}^{b}\right)$ with trace

$$
\begin{aligned}
{ }_{1} X & ={ }_{1}^{h} X+{ }_{1}^{c v} X=\left[{ }_{1}^{h} X\right]+\left[{ }_{1}^{c v} X\right]:=\operatorname{tr}\left({ }_{1} \mathbf{X}\right) \\
& =\operatorname{tr}\left({ }_{1}^{h} X\right)+\operatorname{tr}\left({ }_{1}^{c v} X\right)={ }_{1} \mathbf{X}_{\mu}^{\mu}={ }_{1} X_{i}^{i}+{ }_{1} X_{a}{ }^{a} .
\end{aligned}
$$

Such d-tensors are parameterized as "lifts" of certain $h$ objects to $v$-objects and/or $c v$-objects. The respective formulas are:

$$
\begin{aligned}
\text { for }{ }_{h} e_{k}(\mathbf{X}): & e_{0}\left({ }^{h} X\right)=1, e_{1}\left({ }^{h} X\right)={ }^{h} X, \\
2 e_{2}\left({ }^{h} X\right)= & { }^{h} X^{2}-\left[{ }^{h} X^{2}\right], \\
6 e_{3}\left({ }^{h} X\right)= & { }^{h} X^{3}-3{ }^{h} X\left[{ }^{h} X^{2}\right]+2\left[{ }^{h} X^{3}\right], \\
24 e_{4}\left({ }^{h} X\right)= & { }^{h} X^{4}-6{ }^{h} X^{2}\left[{ }^{h} X^{2}\right]+3\left[{ }^{h} X^{2}\right]^{2} \\
& +8{ }^{h} X\left[{ }^{h} X^{3}\right]-6\left[{ }^{h} X^{4}\right] ; e_{k}\left({ }^{h} X\right)=0 \\
& \text { for } \stackrel{\circ}{k}>4 ;
\end{aligned}
$$$$
\text { for }{ }_{v} e_{k}(\mathbf{X}): \quad e_{0}\left({ }^{v} X\right)=1, e_{1}\left({ }^{v} X\right)={ }^{v} X \text {, }
$$$$
2 e_{2}\left({ }^{v} X\right)={ }^{v} X^{2}-\left[{ }^{v} X^{2}\right], 6 e_{3}\left({ }^{v} X\right)
$$$$
={ }^{v} X^{3}-3{ }^{v} X\left[{ }^{v} X^{2}\right]+2\left[{ }^{v} X^{3}\right]
$$$$
24 e_{4}\left({ }^{v} X\right)={ }^{v} X^{4}-6{ }^{v} X^{2}\left[{ }^{v} X^{2}\right]+3\left[{ }^{v} X^{2}\right]^{2}
$$$$
+8{ }^{v} X\left[{ }^{v} X^{3}\right]-6\left[{ }^{v} X^{4}\right] ; e_{k}\left({ }^{v} X\right)=0
$$$$
\text { for } \stackrel{\circ}{*}>4 \text {; }
$$

for ${ }_{h} e_{k}\left({ }_{1} \mathbf{X}\right): \quad e_{0}\left({ }_{1}^{h} X\right)=1, e_{1}\left({ }_{1}^{h} X\right)={ }_{1}^{h} X$,

$$
\begin{aligned}
& 2 e_{2}\left({ }_{1}^{h} X\right)={ }_{1}^{h} X^{2}-\left[{ }_{1}^{h} X^{2}\right], 6 e_{3}\left({ }_{1}^{h} X\right) \\
& ={ }_{1}^{h} X^{3}-3{ }_{1}^{h} X\left[{ }_{1}^{h} X^{2}\right]+2\left[{ }_{1}^{h} X^{3}\right], \\
& 24 e_{4}\left({ }_{1}^{h} X\right)={ }_{1}^{h} X^{4}-6{ }_{1}^{h} X^{2}\left[{ }_{1}^{h} X^{2}\right]+3\left[{ }_{1}^{h} X^{2}\right]^{2} \\
& +8{ }_{1}^{h} X\left[{ }_{1}^{h} X^{3}\right]-6\left[{ }_{1}^{h} X^{4}\right] ; e_{k}\left({ }_{1}^{h} X\right)=0 \\
& \text { for } k>4 \text {; }
\end{aligned}
$$

for ${ }_{c v} e_{k}\left({ }_{k} \mathbf{X}\right): \quad e_{0}\left({ }_{1}^{c v} X\right)=1, e_{1}\left({ }_{1}^{c v} X\right)={ }_{1}^{c v} X$,

$$
\begin{aligned}
2 e_{2}\left({ }_{1}^{c v} X\right)= & { }_{1}^{c v} X^{2}-\left[{ }_{1}^{c v} X^{2}\right], 6 e_{3}\left({ }_{1}^{c v} X\right) \\
= & { }_{1}^{c v} X^{3}-3{ }_{1}^{c v} X\left[{ }_{1}^{c v} X^{2}\right]+2\left[{ }_{1}^{c v} X^{3}\right], \\
24 e_{4}\left({ }_{1}^{c v} X\right)= & { }_{1}^{c v} X^{4}-6{ }_{1}^{c v} X^{2}\left[{ }_{1}^{c v} X^{2}\right]+3\left[{ }_{1}^{c v} X^{2}\right]^{2} \\
& +8{ }_{1}^{c v} X\left[{ }_{1}^{c v} X^{3}\right]-6\left[{ }_{1}^{c v} X^{4}\right] ; \\
e_{k}\left({ }_{1}^{c v} X\right)= & 0, \stackrel{\circ}{k}>4 .
\end{aligned}
$$

Following [24,68,69,76,106,200-203,207], we state for locally anisotropic gravity theories the

Principle 3.6 -Convention (Actions for MDR-modified massive gravity theories) We introduce the mass-deformed scalar curvatures

$$
\begin{aligned}
\check{R}:= & { }_{s} R+2{ }^{h} \mu^{2}\left(3-\operatorname{tr} \sqrt{h\left(g^{-1} q\right)}-\operatorname{det} \sqrt{h\left(g^{-1} q\right)}\right) \\
& +2{ }^{v} \mu^{2}\left(3-t r \sqrt{v\left(g^{-1} q\right)}-\operatorname{det} \sqrt{v\left(g^{-1} q\right)}\right)
\end{aligned}
$$

and/or

$$
\begin{aligned}
{ }_{1} \check{R}:= & { }_{s} R+2{ }_{1}^{h} \mu^{2}\left(3-\operatorname{tr} \sqrt{h\left({ }_{1} g^{-1}, q\right)}-\operatorname{det} \sqrt{h\left(, g^{-1}, q\right)}\right) \\
& +2 \stackrel{c v}{c v} \mu^{2}\left(3-\operatorname{tr} \sqrt{c v\left({ }_{1} g^{-1}, q\right)}\right. \\
& \left.-\operatorname{det} \sqrt{c v\left({ }_{1} g^{-1}, q\right)}\right),
\end{aligned}
$$

with (MDR-induced, or other variants of $N$-connection structures) horizontal, ${ }^{h} \mu$ and/or ${ }_{1}^{h} \mu$, and (co)vertical, ${ }^{v} \mu$ and ${ }_{1}^{c v} \mu$, masses for locally anisotropic gravitons in respective phase spaces. Using such values, we construct respective $f$-modified Lagrange densities, ${ }^{\mathrm{g} \mu} \mathcal{L}=\mathbf{f}(\check{R})$ and/or ${ }_{1}^{\mathrm{g} \mu} \mathcal{L}=, \mathbf{f}(, \check{R})$, and respective actions on cotangent Lorentz bundles,

$$
\begin{aligned}
& \mathcal{S}={ }^{\mathbf{g} \mu} \mathcal{S}+{ }^{m} \mathcal{S}=\frac{1}{16 \pi} \int \delta u \sqrt{\left|\mathbf{g}_{\alpha \beta}\right|}\left({ }^{\mathbf{g} \mu} \mathcal{L}+{ }^{m} \mathcal{L}\right) \\
& \text { and/or } \\
& { }_{\mathcal{S}} \mathcal{S}={ }_{{ }^{\mathbf{g}} \mu} \mathcal{S}+{ }_{1}^{m} \mathcal{S}=\frac{1}{16 \pi} \int \delta{ }^{\prime} u \sqrt{\left|{ }^{\prime} \mathbf{g}_{\alpha \beta}\right|}\left({ }_{1}^{\mathbf{g} \mu} \mathcal{L}+{ }_{1}^{m} \mathcal{L}\right),
\end{aligned}
$$

where the Lagrange densities for matter fields, ${ }^{m} \mathcal{L}$ and/or ${ }_{1}^{m} \mathcal{L}$, can be taken in as for scalar fields (40), or generalized to YMH and/or fermion configurations.

Performing a $\mathrm{N}$-adapted variational calculus for above introduced Lagrange densities and actions for massive gravity models, with ${ }^{1} \mathbf{f}(\breve{R}):=d \mathbf{f}(\check{R}) / d \check{R}$ and ${ }_{-}^{1} \mathbf{f}(\mid \breve{R}):=$ $d$ |f $\left({ }_{1} \check{R}\right) / d, \check{R}$, we prove:

Consequence 3.4 (Sources in massive gravity with MDRs on (co)tangent bundles) On (co)tangent Lorentz bundles, massive gravity models encoding MDRs are characterized by respective (effective) sources

$$
\begin{aligned}
{ }^{f \mu} \boldsymbol{\Upsilon}_{\mu \nu}= & { }^{g \mu} \boldsymbol{\Upsilon}_{\mu \nu}+{ }^{f} \boldsymbol{\Upsilon}_{\mu \nu}+{ }^{m} \boldsymbol{\Upsilon}_{\mu \nu}, \\
{ }^{g \mu} \boldsymbol{\Upsilon}_{\mu \nu}= & \left.{ }^{g \mu} \Upsilon_{i j},{ }^{g \mu} \boldsymbol{\Upsilon}_{a b}\right), \text { where } \\
{ }^{g \mu} \boldsymbol{\Upsilon}_{i j}= & -2{ }^{h} \mu^{2}\left[3-t r \sqrt{h\left(g^{-1} q\right)}\right. \\
& \left.+\frac{1}{2} \operatorname{det} \sqrt{\left.h\left(g^{-1} q\right)\right)}\right] g_{i j} \\
& +\frac{{ }^{h} \mu^{2}}{2}\left\{q_{i k}\left[\left(\sqrt{h\left(g^{-1} q\right)}\right)^{-1}\right]_{j}^{k}\right. \\
& \left.+q_{i k}\left[\left(\sqrt{h\left(g^{-1} q\right)}\right)^{-1}\right]_{j}^{k}\right\}, \\
{ }^{g \mu} \boldsymbol{\Upsilon}_{a b}= & -2{ }^{v} \mu^{2}\left[3-t r \sqrt{v\left(g^{-1} q\right)}\right. \\
& \left.+\frac{1}{2} \operatorname{det} \sqrt{\left.v\left(g^{-1} q\right)\right)}\right] g_{i j} \\
& +\frac{{ }^{v} \mu^{2}}{2}\left\{q_{a c}\left[\left(\sqrt{v\left(g^{-1} q\right)}\right)^{-1}\right]_{b}^{c}\right.
\end{aligned}
$$




$$
\begin{gathered}
\left.+q_{a c}\left[\left(\sqrt{v\left(g^{-1} q\right)}\right)^{-1}\right]_{b}^{c}\right\}, \\
{ }^{f} \boldsymbol{\Upsilon}_{\mu \nu}=\left(\frac{\mathbf{f}}{2^{1} \mathbf{f}}-\frac{\mathbf{D}^{2}{ }^{1} \mathbf{f}}{{ }^{1} \mathbf{f}}\right) \mathbf{g}_{\mu \nu}+\frac{\mathbf{D}_{\mu} \mathbf{D}_{v}{ }^{1} \mathbf{f}}{{ }^{1} \mathbf{f}} ; \\
{ }^{m} \boldsymbol{\Upsilon}_{\mu \nu}=\frac{1}{2 M_{P}^{2}}{ }^{m} \mathbf{T}_{\mu \nu} ;
\end{gathered}
$$

or (for sources) on cotangent bundles

$$
\begin{aligned}
& { }_{1}^{\mathbf{f} \mu} \Upsilon_{\mu \nu}={ }_{1}^{g \mu} \boldsymbol{\Upsilon}_{\mu \nu}+{ }_{1}^{f} \boldsymbol{\Upsilon}_{\mu \nu}+{ }_{1}^{m} \Upsilon_{\mu \nu}, \\
& { }_{1}^{g \mu} \Upsilon_{\mu \nu}=\left({ }_{1}^{g \mu} \Upsilon_{i j},{ }_{1}^{g \mu} \Upsilon^{a b}\right) \text {, where } \\
& { }_{1}^{g \mu} \Upsilon_{i j}=-2{ }_{1}^{h} \mu^{2}\left[3-t r \sqrt{h\left({ }_{1} g^{-1}{ }_{1} q\right)}\right. \\
& \left.+\frac{1}{2} \operatorname{det} \sqrt{\left.h\left(, g^{-1}, q\right)\right)}\right] g_{i j} \\
& +\frac{{ }_{1}^{h} \mu^{2}}{2}\left\{{ }_{1} q_{i k}\left[\left(\sqrt{h\left(, g^{-1}, q\right)}\right)^{-1}\right]_{j}^{k}\right. \\
& \left.+{ }_{,} q_{i k}\left[\left(\sqrt{h\left(, g^{-1}, q\right)}\right)^{-1}\right]_{j}^{k}\right\} \text {, } \\
& { }_{1}^{g \mu} \Upsilon^{a b}=-2{ }_{1}^{c v} \mu^{2}\left[3-\operatorname{tr} \sqrt{c v\left({ }_{1} g^{-1}{ }_{1} q\right)}\right. \\
& \left.+\frac{1}{2} \operatorname{det} \sqrt{\left.c v\left(, g^{-1}, q\right)\right)}\right], g_{i j} \\
& +\frac{{ }^{c} v}{2} \mu^{2}\left\{{ }_{1} q^{a c}\left[\left(\sqrt{c v\left(, g^{-1}, q\right)}\right)^{-1}\right]_{c}^{b}\right. \\
& \left.+{ }_{1} q^{a c}\left[\left(\sqrt{c v\left(, g^{-1}, q\right)}\right)^{-1}\right]_{c}^{b}\right\},
\end{aligned}
$$

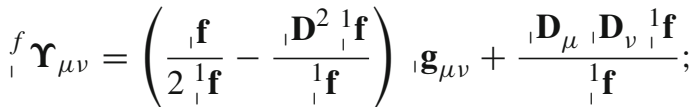

$$
\begin{aligned}
& { }_{1}^{m} \Upsilon_{\mu \nu}=\frac{1}{2 M_{P}^{2}}{ }_{1}^{m} \mathbf{T}_{\mu \nu} .
\end{aligned}
$$

In 4-d massive MGTs $[24,76,106]$, there are considered values of type $S^{i}{ }_{j}=g^{i k} \eta_{\overline{i j}} \mathbf{e}_{k} S^{\bar{j}} \mathbf{e}_{j} S^{\bar{j}}$, with the Minkowski metric $\eta_{\bar{i} j}=\operatorname{diag}(1,1,1,-1)$, generated by four scalar Stükelberg fields $s^{\bar{j}}$, which is necessary for restoring the diffeomorphism invariance. Similar values can be considered for fiducial fields $\mathbf{q}=\left\{, \mathbf{q}_{\alpha \beta}\right\}$ and/or ${ }_{1} \mathbf{q}=\left\{{ }_{1} \mathbf{q}_{\alpha \beta}\right\}$ for massive and bi-metric locally anisotropic theories when the $\mathrm{St}$ ükelberg fields are introduced also on (co)fiber spaces, see details and examples in [68,200-203,207].

The sources of (effective) matter fields are approximated (for instance, for quantum fluctuations or certain summarized contributions) to (effective) cosmological constants. Following Principle 3.4, we consider
Assumption 3.2 (Effective cosmological constants in massive gravity on (co)tangent bundles) Generalized sources for matter fields (including possible effective sources defined by distortion tensors of d-connections) can be approximated by respective cosmological constants $\Lambda$ and/or ${ }_{1} \Lambda$,

${ }^{f \mu} \Upsilon^{\beta}{ }_{\gamma}={ }^{f \mu} \Lambda \delta^{\beta}{ }_{\gamma}$ and/or ${ }_{1}^{f \mu} \Upsilon_{\gamma}^{\beta}{ }_{\gamma}={ }_{1}^{f \mu} \Lambda \delta_{\gamma}^{\beta}$,

when, correspondingly, ${ }^{f \mu} \boldsymbol{\Upsilon}_{\mu \nu}={ }^{g \mu} \boldsymbol{\Upsilon}_{\mu \nu}+{ }^{f} \boldsymbol{\Upsilon}_{\mu \nu}+{ }^{m} \boldsymbol{\Upsilon}_{\mu \nu}$ and/or ${ }^{f \mu} \Upsilon_{\mu \nu}={ }_{1}^{g \mu} \boldsymbol{\Upsilon}_{\mu \nu}+{ }_{1}^{f} \boldsymbol{\Upsilon}_{\mu \nu}+{ }_{1}^{m} \Upsilon_{\mu \nu}$ considering that such sources are related to relations of additivity,

$$
\begin{aligned}
{ }^{f \mu} \Lambda= & { }^{g \mu} \Lambda+{ }^{f} \Lambda+{ }^{m} \Lambda \text { and } / \text { or }{ }_{1}^{f \mu} \Lambda={ }_{1}^{g \mu} \Lambda \\
& +{ }_{1}^{f} \Lambda+{ }_{1}^{m} \Lambda .
\end{aligned}
$$

Bi-metric and/or massive gravity LC-configurations can be extracted by imposing additional (non)holonomic constraints when $\mathbf{D}_{\mathcal{T}=0} \rightarrow \nabla$.

\subsubsection{Lagrange densities for short-range locally anisotropic gravity with MDRs and LIV}

In [11] (see also references therein), a systematic study of LIVs for dimensions dim $>5$ (with explicit formulas for a class of effective MGTs of dimensions $\operatorname{dim}=4,5,6$ ) was initiated. In this subsection, we elaborate on toys models of Lagrange-Hamilton gravity theories of dimension $\operatorname{dim}=$ $3+3$, with signatures of local metrics of type $(++-;++-)$, and indices running values $i, j, \ldots=1,2,3$ and $a, b, \ldots=$ $4,5,6$, for $\alpha=(i, a), \beta=(j, b), \ldots$

Following Principle 3.4, we change the LC-connection into a metric compatible d-connection completely determined by the same metric structure and formulate:

Principle 3.7 -Convention (Lagrange densities for MDRmodified short-range gravity and LIV) The Lagrange densities of underlying actions for effective gravity theory with short-range, sr, locally anisotropic interactions with spontaneous LIVs are determined by such sums:

$$
\begin{aligned}
& { }^{\text {sr }} \mathcal{L}={ }^{\mathbf{g}} \mathcal{L}+{ }^{m} \mathcal{L}+{ }^{k} \mathcal{L}+{ }^{l v} \mathcal{L} \text {, where } \\
& { }^{\mathbf{g}} \mathcal{L}={ }^{s} \mathbf{R}-2 \Lambda,{ }^{m} \mathcal{L}={ }^{m} \mathcal{L} \text { see }(40), \\
& { }^{k} \mathcal{L}=\text { is for the dynamics of fields triggering } \\
& \text { spontaneous LIVs } \\
& { }^{l v} \mathcal{L}={ }^{l v} \mathcal{L}_{[4]}+{ }^{l v} \mathcal{L}_{[5]}+{ }^{l v} \mathcal{L}_{[6]}+\cdots, \text { for } \\
& { }^{l v} \mathcal{L}_{[4]}=\kappa_{\alpha \beta \gamma \delta}^{[4]}(x, y) \mathbf{R}^{\alpha \beta \gamma \delta}[\mathbf{D}], \\
& { }^{l v} \mathcal{L}_{[5]}=\kappa_{\alpha \beta \gamma \delta \tau}^{[5]}(x, y) \mathbf{D}^{\tau} \mathbf{R}^{\alpha \beta \gamma \delta}, \\
& { }^{l v} \mathcal{L}_{[6]}=\frac{1}{2}{ }^{1} \kappa_{\alpha \beta \gamma \delta \tau \nu}^{[6]}(x, y)\left(\mathbf{D}^{\tau} \mathbf{D}^{v}+\mathbf{D}^{v} \mathbf{D}^{\tau}\right) \mathbf{R}^{\alpha \beta \gamma \delta} \\
& +{ }^{2} \kappa_{\alpha \beta \gamma \delta \tau \nu \lambda \theta}^{[6]}(x, y) \mathbf{R}^{\tau \nu \lambda \theta} \mathbf{R}^{\alpha \beta \gamma \delta} \text {; }
\end{aligned}
$$


and/or

$$
\begin{aligned}
& { }_{1}^{\mathbf{s r}} \mathcal{L}={ }_{1}^{\mathrm{g}} \mathcal{L}+{ }_{1}{ }^{\mathrm{lv}} \mathcal{L}+{ }_{1}^{k} \mathcal{L}+{ }_{1}^{m} \mathcal{L} \\
& { }_{1}^{\mathbf{g}} \mathcal{L}={ }_{1}^{s} \mathbf{R}-2{ }_{1} \Lambda,{ }_{1}^{m} \mathcal{L}={ }_{1}^{m} \mathcal{L} \text { see }(40), \\
& { }_{1}^{k} \mathcal{L}=\text { is for the dynamics of fields triggering } \\
& \text { spontaneous LIVs } \\
& { }_{1}^{l v} \mathcal{L}={ }_{1}^{l v} \mathcal{L}_{[4]}+{ }_{1}^{l v} \mathcal{L}_{[5]}+{ }_{1}^{\text {lv }} \mathcal{L}_{[6]}+\cdots \text {, for } \\
& { }_{1}^{l v} \mathcal{L}_{[4]}={ }_{1} \kappa_{\alpha \beta \gamma \delta}^{[4]}(x, p){ }_{,} \mathbf{R}^{\alpha \beta \gamma \delta}\left[{ }_{,}, \mathbf{D}\right], \\
& { }_{1}^{l v} \mathcal{L}_{[5]}={ }_{1}{ }_{\alpha}{ }_{\alpha \beta \gamma \delta \tau}^{[5]}(x, p){ }_{1} \mathbf{D}^{\tau}{ }_{,} \mathbf{R}^{\alpha \beta \gamma \delta \delta}, \\
& { }_{1}^{l v} \mathcal{L}_{[6]}=\frac{1}{2}{ }_{1}^{1} \kappa_{\alpha \beta \gamma \delta \tau \nu}^{[6]}(x, p)\left({ }_{1} \mathbf{D}^{\tau}{ }_{1} \mathbf{D}^{\nu}+{ }_{,} \mathbf{D}^{\nu},{ }_{1} \mathbf{D}^{\tau}\right){ }_{1} \mathbf{R}^{\alpha \beta \gamma \delta} \\
& +{ }_{1}^{2} \kappa_{\alpha \beta \gamma \delta \tau \nu \lambda \theta}^{[6]}(x, p){ }_{1} \mathbf{R}^{\tau \nu \lambda \theta}{ }_{1} \mathbf{R}^{\alpha \beta \gamma \delta} \text {, }
\end{aligned}
$$

where $\kappa$-coefficients are taken as in [11] (see also references therein) but with $h-v$-and/or $h-c v$-decompositions.

The $h$-components of above formulas transform into holonomic ones for $\mathbf{D}_{\mathcal{T}=0} \rightarrow \nabla$.

Performing respective $\mathrm{N}$-adapted variational calculuses for actions

$$
\begin{aligned}
& { }^{\mathbf{s r}} \mathcal{S}={ }^{\mathbf{g}} \mathcal{S}+{ }^{m} \mathcal{S}+{ }^{k} \mathcal{S}+{ }^{l v} \mathcal{S} \\
& =\frac{1}{16 \pi G_{\text {dim }}} \int \delta u \sqrt{\left|\mathbf{g}_{\alpha \beta}\right|}\left({ }^{\mathbf{g}} \mathcal{L}+{ }^{m} \mathcal{L}+{ }^{k} \mathcal{L}+{ }^{l v} \mathcal{L}\right) \\
& \text { and/or } \\
& { }_{1}^{\mathbf{s r}} \mathcal{S}={ }_{1}^{\mathrm{g}} \mathcal{S}+{ }_{1}^{m} \mathcal{S}+{ }_{1}^{k} \mathcal{S}+{ }_{1}{ }_{1} \mathcal{S} \\
& =\frac{1}{16 \pi^{\prime} G_{\operatorname{dim}}} \int \delta^{\prime} u \sqrt{\left|{ }^{\prime} \mathbf{g}_{\alpha \beta}\right|}\left({ }_{1}^{\mathbf{g}} \mathcal{L}+{ }_{1}^{m} \mathcal{L}+{ }_{1}^{k} \mathcal{L}+{ }_{1}^{l v} \mathcal{L}\right),
\end{aligned}
$$

with conventional Newton constants $G_{\operatorname{dim}}$ and ${ }^{\prime} G_{\operatorname{dim}}$ one proves:

Consequence 3.5 (Effective matter energy-momentum dtensors in short-range gravity on (co)tangent Lorentz bundles) The symmetric energy-momentum d-tensors for matter fields and sources induced by LIV on (co)tangent bundles derived for respective Lagrange densities (52) are computed

$$
\begin{aligned}
{ }^{s r} \mathbf{T}_{\beta \delta}= & { }^{m} \mathbf{T}_{\beta \delta}+{ }^{l v} \mathbf{T}_{\beta \delta}, \text { for }{ }^{m} \mathbf{T}_{\beta \delta} \text { as in (41) and } \\
{ }^{l v} \mathbf{T}_{\beta \delta}= & \frac{1}{4 \pi G_{\operatorname{dim}}} \widehat{\bar{s}}^{\alpha \beta} \mathbf{E}_{\alpha(\mu \nu) \beta}-\frac{1}{16 \pi G_{\operatorname{dim}}} \widehat{\bar{u}} \mathbf{E}_{\mu \nu} \\
& +\frac{a}{8 \pi G_{\operatorname{dim}}}{ }^{1} \bar{\kappa}_{\alpha(\mu \nu) \beta \gamma \delta}^{[6]} \mathbf{e}^{\alpha} \mathbf{e}^{\beta} \mathbf{R}^{\gamma \delta} \\
& +\frac{1}{2 \pi G_{\operatorname{dim}}}{ }^{2} \bar{\kappa}_{\alpha(\mu \nu) \beta \gamma \delta \epsilon \zeta}^{[6]} \mathbf{e}^{\alpha} \mathbf{e}^{\beta} \mathbf{R}^{\gamma \delta \epsilon \zeta},
\end{aligned}
$$

and/or

$$
\begin{aligned}
& { }_{1}^{s r} \mathbf{T}_{\beta \delta}={ }_{1}^{m} \mathbf{T}_{\beta \delta}+{ }_{1}{ }^{v} \mathbf{T}_{\beta \delta} \text {, for }{ }_{1}^{m} \mathbf{T}_{\beta \delta} \text { as in (41) and } \\
& { }_{1}^{l v} \mathbf{T}_{\beta \delta}=\frac{1}{4 \pi{ }^{\prime} G_{\operatorname{dim}}}{ }^{\widehat{s}}{ }^{\alpha \beta}{ }^{\prime} \mathbf{E}_{\alpha(\mu \nu) \beta}-\frac{1}{16 \pi G_{\operatorname{dim}}}{ }^{\prime} \widehat{\bar{u}}^{\prime} \mathbf{E}_{\mu \nu} \\
& +\frac{1 a}{8 \pi G_{\operatorname{dim}}},{ }_{1} \bar{\kappa}_{\alpha(\mu \nu) \beta \gamma \delta}^{[6]} \mathbf{e}^{\alpha}{ }^{\prime} \mathbf{e}^{\beta}{ }^{\prime} \mathbf{R}^{\gamma \delta} \\
& +\frac{1}{2 \pi{ }^{\prime} G_{\operatorname{dim}}}{ }^{2} \bar{\kappa}_{\alpha(\mu \nu) \beta \gamma \delta \epsilon \zeta}^{[6]}{ }^{\prime} \mathbf{e}^{\alpha} \mathbf{e}^{\beta}{ }^{\beta} \mathbf{R}^{\gamma \delta \epsilon \zeta} .
\end{aligned}
$$

The coefficients and operators in (53) are computed in certain forms which are similar to formula (6) in $[11]^{8}$ when $\partial^{\alpha} \rightarrow \mathbf{e}^{\alpha}$ and $\nabla \rightarrow \mathbf{D}$ on $T \mathbf{V}$.Here we note that the double dual of the Riemannian d-tensor $\mathbf{E}_{\alpha \beta \gamma \delta}:=$ $\frac{1}{4} \epsilon_{\alpha \beta \kappa \lambda} \epsilon_{\gamma \delta \mu \nu} \mathbf{R}^{\kappa \lambda \mu \nu}$, the symmetrization of indices is denoted by " $(\alpha \beta)$ " and $a$ is an integration constant. Similar values for $T^{*} \mathbf{V}$ are labeled by " "”.

The sources of (effective) matter fields are approximated (for instance, for quantum fluctuations or certain summarized contributions) to (effective) cosmological constants. Following Principle 3.4, we consider

Assumption 3.3 (Effective cosmological constants for short-range locally anisotropic interactions) Generalized (effective) sources for matter and fields (including possible effective sources defined by distortion tensors of $\mathrm{d}$ connections) in short-range locally anisotropic gravity can be approximated by respective cosmological constants ${ }^{s r} \Lambda$ and/or ${ }_{\perp}^{s r} \Lambda$, when effective sources

$$
{ }^{s r} \Upsilon^{\beta}{ }_{\gamma}={ }^{s r} \Lambda \delta_{\gamma}^{\beta} \text { and/or } \quad{ }_{1}^{s r} \Upsilon^{\beta}{ }_{\gamma}={ }_{1}^{s r} \Lambda \delta^{\beta}{ }_{\gamma}
$$

are taken respectively ${ }^{s r} \Upsilon_{\mu \nu}={ }^{m} \Upsilon_{\mu \nu}+{ }^{l v} \Upsilon_{\mu \nu}$ and/or ${ }_{1}^{s r} \Upsilon_{\mu \nu}={ }_{1}^{m} \Upsilon_{\mu \nu}+{ }_{1}^{l v} \Upsilon_{\mu \nu}$, for relations of additivity

${ }^{s r} \Lambda={ }^{m} \Lambda+{ }^{l v} \Lambda$ and ${ }_{1}^{s r} \Lambda={ }_{1}^{m} \Lambda+{ }_{1}^{l v} \Lambda$.

Short-range LC-configurations (in general, parameterized by off-diagonal metrics) can be extracted by imposing additional (non)holonomic constraints when $\mathbf{D}_{\mathcal{T}=0} \rightarrow \nabla$. The constructions can be performed in similar forms, for instance, if respective energy-momentum tensors for YMH fields are considered instead of ${ }^{m} \mathbf{T}_{\beta \delta}$, or there are studied massive gravity effects and bi-metric structures.

\subsubsection{The equations of motion and nonholonomic conservation laws for (effective) sources}

The conservation laws

$\nabla^{i}\left(R_{i j}-\frac{1}{2} g_{i j} R\right)=0$ and $\nabla_{i} T^{i j}=0$

in GR are consequences of the Bianchi relations. Such laws involve the idea that the Einstein's equations actually imply the geodesic hypothesis when the world lines of test bodies are geodesics of the spacetime metric. However, it should be noted that bodies which are "large" enough to feel the tidal forces of the gravitational field will deviate from geodesic motion. Such deviations may be caused by internal symmetries and (non)linear interactions and certain nonholonomic constraints on the dynamics of gravitational and matter fields.

\footnotetext{
${ }^{8}$ Readers may see that paper and references therein for details on symmetries of LIV coefficients, definition of operators and sources etc.
} 
Nevertheless, the equations of motion of "large and/or complex structure" bodies in GR also can be found from the condition $\nabla_{a} T^{a b}=0$.

For a Finsler d-connection $\mathbf{D}$ (even it can be chosen to metric compatible, $\mathbf{D}_{\alpha} \mathbf{g}^{\beta \gamma}=0$ ), we have $\mathbf{D}_{\alpha} \mathbf{T}^{\alpha \beta} \neq 0$, which is a consequence of non-symmetry of the Ricci and Einstein d-tensors; see explanations for formulas (30) and (31) and Definition-Theorems 2.9 and generalized Bianchi identities. Such a property is also related to nonholonomic constraints on the dynamics of Finsler gravitational fields. It is not surprising that the "covariant divergence" of (effective) matter sources does not vanish even for canonical d-connections $\widehat{\mathbf{D}}$ and/or $\widetilde{\mathbf{D}}$ (on nonholonomic manifolds and/or (co)tangent bundles) see formulas (32) and (33). In such cases, the conservation law became more sophisticate because of nonholonomic constraints and differences between the autoparallels of $\mathbf{D}$ and/or ' $\mathbf{D}$ and nonlinear geodesic (semi-spray) equations (15). Nevertheless it is possible to compute effective nonholonomic tidal forces of locally anisotropic gravitational fields using distorting relations of type $\mathbf{D}=\nabla-\mathbf{Z}$ (28), see Lemma 2.2 and, for canonical distortions, formulas (34).

Assumption 3.4 (Energy-momentum d-tensors for (non) massive gravity theories on (co)tangent Lorentz bundles) The (effective) energy momentum d-tensors on (co)tangent Lorentz bundles are defined by all possible components (41), (45), (46), (50), (51) and (53) constructed by respective geometric principles and $\mathrm{N}$-adapted variational calculuses,

$$
\begin{aligned}
\mathbf{T}_{\alpha \beta}= & { }^{\phi} \mathbf{T}_{\alpha \beta}+{ }^{A} \mathbf{T}_{\alpha \beta}+{ }^{H} \mathbf{T}_{\alpha \beta}+{ }^{g \mu} \mathbf{T}_{\alpha \beta} \\
& +{ }^{f} \mathbf{T}_{\alpha \beta}+{ }^{l v} \mathbf{T}_{\alpha \beta}+\ldots \\
{ }_{1} \mathbf{T}_{\alpha \beta}= & { }_{1}{ }_{1} \mathbf{T}_{\alpha \beta}+{ }_{1}^{A} \mathbf{T}_{\alpha \beta}+{ }_{1}{ }_{1} \mathbf{T}_{\alpha \beta}+{ }_{1}{ }^{g \mu} \mathbf{T}_{\alpha \beta} \\
& +{ }_{1}{ }_{1} \mathbf{T}_{\alpha \beta}+{ }_{1}{ }_{1}^{l v} \mathbf{T}_{\alpha \beta}+\ldots
\end{aligned}
$$

Dots in these formulas contain possible other types contributions of locally anisotropic spinor fields, from (super) string gravity, noncommutative deformations, fractional, diffusion and kinetic processes etc., see examples in Refs. [13,23,68-70,147, 149, 150,152,153,161,167, 169, $170,173,179,189,192,206,207,209]$. The sources in such works were considered in the bulk for nonholonomic manifolds and tangent bundles. But analogy, the constructions can re-defined for cotangent Lorentz bundles. In this work, we omit cumbersome formulas for the Bianchi identities and conservation laws with nonholonomic constraints written in variables $(\mathbf{g}, \mathbf{N}, \mathbf{D})$ and/or ( 'g, 'N, 'D).

We note that the Bianchi identities for some data $(\mathbf{g}, \mathbf{N}, \widehat{\mathbf{D}})$ can be computed by introducing nonholonomic deformations $\nabla=\widehat{\mathbf{D}}-\widehat{\mathbf{Z}}$ into the standard relations $\nabla^{\alpha}\left(R_{\alpha \beta}-\frac{1}{2} g_{\alpha \beta} R\right)=0$ and $\nabla^{\alpha} T_{\alpha \beta}=0$. Even, in general, $\widehat{\mathbf{D}}^{\alpha} \mathbf{T}_{\alpha \beta}=\mathbf{Q}_{\beta} \neq 0$, such a $\mathbf{Q}_{\beta}[\mathbf{g}, \mathbf{N}]$ is completely defined by the d-metric and chosen $\mathrm{N}$-connection structure. This is a consequence of the nonholonomic structure. A similar "problem" exists in Lagrange mechanics with non-integrable constraints when the standard conservation laws do not hold true. A new class of effective variables can be introduced using Lagrange multiples.

Principle 3.8 (Nonholonomic deformations by MDRs of conservation laws on (co)tangent Lorentz bundles) Postulating for respective LC-connections $\nabla$ and/or ${ }^{\prime} \nabla$ on (co)tangent Lorentz bundles conservation laws of type

$$
\begin{aligned}
& \nabla^{\alpha}\left(R_{\alpha \beta}-\frac{1}{2} g_{\alpha \beta} R\right)=0 \text { and } \nabla^{\alpha} T_{\alpha \beta}=0 ; \text { and/or } \\
& { }^{\prime} \nabla^{\alpha}\left({ }^{\prime} R_{\alpha \beta}-\frac{1}{2}{ }^{\prime} g_{\alpha \beta}{ }^{\prime} R\right)=0 \text { and }{ }^{\prime} \nabla^{\alpha}{ }^{\prime} T_{\alpha \beta}=0,
\end{aligned}
$$

we define unique nonholonomic conservation laws

$\mathbf{D}^{\alpha} \mathbf{T}_{\alpha \beta}=\mathbf{Q}_{\beta} \neq 0$ and/or ${ }^{\prime} \mathbf{D}^{\alpha}{ }^{\prime} \mathbf{T}_{\alpha \beta}={ }^{\prime} \mathbf{Q}_{\beta} \neq 0$,

with d-tensors $\mathbf{Q}_{\beta}$ and/or ${ }^{\prime} \mathbf{Q}_{\beta}$ uniquely determined by respective data with unique distortion relations $(\mathbf{g}, \mathbf{N}, \mathbf{D}=$ $\nabla-\mathbf{Z})$ and/or $\left({ }^{\prime} \mathbf{g},{ }^{\prime} \mathbf{N},{ }^{\prime} \mathbf{D}={ }^{\prime} \nabla-{ }^{\prime} \mathbf{Z}\right)$.

Nonholonomic deformations of conservation laws (56) are similar to modifications of conservation laws in nonholonomic Lagrange and/or Hamilton mechanics. The motion of probing point particles in phase spaces with MDRs are modeled by canonical data for Finsler-Lagrange-Hamilton spaces with respective d-connections (32) and (33) and canonical distortions (34). Nonholonomic canonical deformations $\widetilde{\mathbf{D}}^{\alpha} \widetilde{\mathbf{T}}_{\alpha \beta}=\widetilde{\mathbf{Q}}_{\beta} \neq 0$ and ${ }^{\prime} \widetilde{\mathbf{D}}^{\alpha}{ }^{\prime} \widetilde{\mathbf{T}}_{\alpha \beta}={ }^{\prime} \widetilde{\mathbf{Q}}_{\beta} \neq 0$ are considered also in almost symplectic classical and (deformation) quantum models, commutative and noncommutative, of gravity. Finally, we note that Principle 3.8 has to be re-formulated and stated in model dependent forms for theories with multi-metric structure, in metric-affine spaces with nontrivial nonmetricity, nonlocal interactions etc.

\section{Modified field equations with MDRs and Lagrange-Hamilton gravity}

The goal of this section is to formulate the gravitational and matter field equations in MGTs with MDRs and LIVs. Such systems of nonlinear PDEs can be derived in abstract and/or $\mathrm{N}$-adapted (in general, coordinate free) forms following Principles 3.3 and 3.4 and applying geometric and variational methods using Lagrange densities on (co)tangent Lorentz bundles postulated in previous section.

4.1 Generalized Einstein equations on nonholonomic (co)tangent Lorentz bundles

We shall analyse modified gravitational field equations for d-metrics (13) and (14) (see Assumption 2.3 and equivalent to off-diagonal metrics (20)) and metric compatible d-connections. The (effective) matter sources $\Upsilon_{\beta \gamma}:=$ 
$\varkappa\left(\mathbf{T}_{\alpha \beta}-\frac{1}{2} \mathbf{g}_{\alpha \beta} \mathbf{T}\right)$ and/or ${ }^{\prime} \Upsilon_{\beta \gamma}:=\varkappa\left({ }^{\prime} \mathbf{T}_{\alpha \beta}-\frac{1}{2}{ }^{\prime} \mathbf{g}_{\alpha \beta}{ }^{\prime} \mathbf{T}\right)$ are determined by energy-momentum d-tensors of type (55), see Assumption 3.4. Such sources are defined both by distortion d-tensors of respective d-connections and generalized energy-momentum d-tensors.

\subsubsection{Nonholonomic Einstein equations with general (effective) sources}

Following a N-adapted variational calculus for data $(T \mathbf{V}$, $\mathbf{N}, \mathbf{g}, \mathbf{D})$ and source $\Upsilon_{\beta \gamma}$, we motivate and prove:

Principle 4.1 -Theorem (Nonholonomic modifications of Einstein equations on tangent Lorentz bundles) The modified Einstein equations for the Ricci d-tensor (30) corresponding to some metric compatible data $(\mathbf{g}, \mathbf{D g}=0)$ and an effective source $\Upsilon_{\beta \gamma}$ computed for (55) on a tangent Lorentz, bundle TV are

$\mathbf{R}_{\alpha \beta}[\mathbf{D}]=\Upsilon_{\alpha \beta}$.

Considering distortion d-tensors, $\mathbf{D} \rightarrow \check{D}=\mathbf{D}+\mathbf{Z}$ determined in unique forms by data $(\mathbf{N}, \mathbf{g})$ following certain geometric principles, the equations (57) can be re-defined for metric non-compatible d-connections, $\check{\mathbf{D}} g \neq 0$. Such theories determined by different types of (effective) sources $\Upsilon_{\alpha \beta}$, and examples of exact solutions, are studied in details in monograph [161]. Using frame transforms $\mathbf{g}_{\alpha \beta}=e_{\alpha}^{\alpha^{\prime}} e_{\beta}^{\beta^{\prime}} g_{\alpha^{\prime} \beta^{\prime}}$ and distortion relations of type (34), we prove

Corollary 4.1 (Canonical form of locally anisotropic Einstein equations on tangent bundles) The modified Einstein equations (57) on TV can be written equivalently for a canonical d-connection $\widehat{\mathbf{D}}$

$\widehat{\mathbf{R}}_{\alpha \beta}[\widehat{\mathbf{D}}]=\widehat{\Upsilon}_{\alpha \beta}$.

Imposing additional (non)holonomic constraints for zero torsion, $\widehat{\mathbf{D}}_{\mid \widehat{T}=0}=\nabla$, we extract LC-configurations when

$\widehat{\mathcal{T}}=0$.

The equations (59) can be solved in exact form for configurations subjected to the conditions (36).

Remark 4.1 (Nonholonomic Lagrange-Finsler variables for locally anisotropic Einstein equations) The system of nonlinear PDEs (57) and (59) can be considered on (pseudo) Riemannian manifolds with nonholonomic fibered $h$-and $v$-structures. Prescribing N-connection structures, we can reformulate the GR theory and the Einstein equations using data $(\mathbf{g}, \nabla)$ and/or in terms of nonholonomic variables with data $(\mathbf{g}, \widehat{\mathbf{D}})$.

Variables $(\mathbf{g}, \widehat{\mathbf{D}})$ with corresponding parameterizations for $2+2+2+\cdots$ splitting of spacetimes and phase spaces play a preferred role in elaborating the AFDM for decoupling and integrating (modified) Einstein equations, see Refs. $[9,68,69,151,153,158-165,168,169,188-$ 190,196,198,199,201-203]. Certain classes of generic offdiagonal solutions can be constructed in explicit form for the canonical data $(\widetilde{\mathbf{g}}, \widetilde{\mathbf{D}})[182,183,186]$ and (in almost Kähler variables) for performing deformation quantization of gravity theories $[8,172,174,177,184,197,206]$.

\subsubsection{Gravitational equations on Lorentz cotangent bundles}

For geometric data $\left(\mathbf{T}^{*} \mathbf{V},{ }^{\prime} \mathbf{N},{ }^{\prime} \mathbf{g},{ }^{\prime} \mathbf{D}\right)$ and prescribed source ${ }^{\prime} \Upsilon_{\beta \gamma}$, we derive

Principle 4.2 -Theorem (Nonholonomic modifications of Einstein equations on cotangent Lorentz bundles) On $\mathbf{T}^{*} \mathbf{V}$, the modified Einstein equations, when ( $\mathbf{g}$, ' $\mathbf{D}$ ' $\mathbf{g}=0$ ), an effective source ' $\Upsilon_{\alpha \beta}$ is computed for d-tensors (55) and Ricci d-tensor (31) are

$$
{ }^{\prime} \mathbf{R}_{\alpha \beta}\left[{ }^{\prime} \mathbf{D}\right]={ }^{\prime} \Upsilon_{\alpha \beta} \text {. }
$$

Considering distortion d-tensors, ${ }^{\prime} \mathbf{D} \rightarrow{ }^{\prime} \check{\mathbf{D}}={ }^{\prime} \mathbf{D}+{ }^{\prime} \mathbf{Z}$ and nonholonomic deformations, we prove

Corollary 4.2 (Canonical form of locally anisotropic Einstein equations on cotangent bundles) The analog of modified Einstein equations (57) on $T \mathbf{V}$ can be derived on $T^{*} \mathbf{V}$ for a canonical d-connection ' $\widehat{\mathbf{D}}$,

$$
{ }^{\prime} \widehat{\mathbf{R}}_{\alpha \beta}\left[{ }^{'} \widehat{\mathbf{D}}\right]={ }^{\prime} \widehat{\Upsilon}_{\alpha \beta} \text {. }
$$

We extract LC-configurations from solutions of such equations if there are imposed additional zero-torsion constraints (37).

Remark 4.2 (Nonholonomic Hamilton-Cartan variables for locally anisotropic Einstein equations) The system of nonlinear PDEs (60) can be re-written equivalently in terms of nonholonomic canonical variables with data $(' \mathbf{g}$, ' $\widehat{\mathbf{D}})$, or ( $\left.\widetilde{\mathbf{g}}, \mathbf{I}^{\mathbf{D}}\right)$. Such equations can be used for constructing generic off-diagonal solutions, or for performing deformation quantization.

In general, theories with gravitational field equations (57) are different from theories determined by systems of type (60) because the corresponding phase spaces and d-connection and d-metric structures are different. Some subclasses of solutions can be transformed mutually in equivalent forms for different theories if canonical variables with $L$-duality are introduced.

\subsection{Modified Einstein equations for pseudo Lagrange-Hamilton spaces}

Using geometric and $\mathrm{N}$-adapted variational methods, we can derive gravitational field equations for different models of Lagrange and Hamilton gravity theory respectively 
constructed on (co)tangent Lorentz bundles. If the geometric objects and fundamental field equations are written in canonical variables, there are obtained systems of nonlinear PDEs containing forth and higher order partial derivatives of generating functions. This makes technically impossible to find physically important exact solutions using standard analytic methods for some reduced nonlinear systems on ODEs. Nevertheless, we can construct very general classes of exact solutions in Einstein-Finsler/-Lagrange/-Hamilton theories considering nonholonomic frame transforms and distortion of connections to certain configurations with decoupling and general integrability properties. In this section, the gravitational field equations are formulated in terms of canonical, i.e. "tilde", variables because such equations can be re-defined in straightforward forms in almost symplectic variables. Such almost Kähler models are important for geometric/deformation quantization and in order to study locally anisotropic kinetic and stochastic processes on relativistic curved spacetimes and phase space generalizations [148, 153-155, 172,174, 177, 178, 184, 195, 197].

\subsubsection{Einstein-Finsler-Lagrange gravity}

We consider canonical d-metric, $\widetilde{\mathbf{g}}(18), \mathrm{N}$-connection, $\widetilde{\mathbf{N}}$, see Theorem (2.2), and d-connection, $\widetilde{\mathbf{D}}$ (32), structures (all defined by a Lagrange generating function $\widetilde{L}$, which can be determined by a MDR (1)). Possible sources $\widetilde{\Upsilon}_{\beta \gamma}$ are prescribed by energy-momentum d-tensors of type (55), see Assumption 3.4, being compatible and constructed by analogy to GR but for such canonical data. In such nonholonomic variables, the Principle-Theorem 4.1 transforms into

Principle 4.3 -Corollary (Generalized canonical Einstein equations for Lagrange gravity) For the canonical data $(\widetilde{L}, \widetilde{\mathbf{N}}, \widetilde{\mathbf{g}}, \widetilde{\mathbf{D}})$, the gravitational field equations for the Einstein-Lagrange phase spaces are

$\widetilde{\mathbf{R}}_{\alpha \beta}[\widetilde{\mathbf{D}}]=\widetilde{\Upsilon}_{\alpha \beta}$.

Considering distortion d-tensors of type $\widetilde{\mathbf{D}} \rightarrow \check{\mathbf{D}}=\widetilde{\mathbf{D}}+$ $\mathbf{Z}$ determined in unique forms by ( $\widetilde{\mathbf{N}}, \widetilde{\mathbf{g}})$, the equation (62) can be re-defined for metric non-compatible d-connections, $\check{\mathbf{D}} \widetilde{\mathbf{g}} \neq 0$. For instance, we can consider the Chern $[16,41]$ or Berwald [20,21] d-connections. Such constructions result in systems of PDEs which can not be integrated in general forms and with ambiguities in introducing spinor fields on pseudo Lagrange spaces, see critics $[185,194]$. It is more efficient to consider frame transforms correlated to distortions to the canonical d-connection, $\widetilde{\mathbf{D}} \rightarrow \widehat{\mathbf{D}}=\widetilde{\mathbf{D}}+\widehat{\mathbf{Z}}$. In result, we prove

Corollary 4.3 (A general integrable canonical form of locally anisotropic Einstein equations on tangent bundles) The modified Einstein equations (62) for Einstein-Lagrange phase spaces can be written equivalently using the canon- ical d-connection $\widehat{\mathbf{D}}$, see Eq. (58), with re-defined sources, $\widetilde{\Upsilon}_{\beta \gamma} \rightarrow \widehat{\Upsilon}_{\beta \gamma}$.

We conclude that the gravitational field equations for the Einstein-Lagrange phase space geometry can be transformed into certain systems of nonlinear PDEs with decoupling properties. In particular, such transforms can be considered for pseudo Finsler spaces with $L=F^{2}$ as in Example 2.4.

\subsubsection{Einstein-Hamilton gravity}

The constructions for Einstein-Lagrange phase spaces can reproduced on cotangent Lorentz bundles taking canonical data for Hamilton geometries. In result, the PrincipleTheorem 4.1 transforms into

Principle 4.4 -Corollary (Generalized canonical Einstein equations for Hamilton gravity) For canonical data $(\widetilde{H}, ' \widetilde{\mathbf{N}}$, ' $\widetilde{\mathbf{g}}, \quad \widetilde{\mathbf{D}})$, the gravitational field equations on the EinsteinHamilton phase spaces are written

$$
{ }^{\prime} \widetilde{\mathbf{R}}_{\alpha \beta}\left[{ }^{\prime} \widetilde{\mathbf{D}}\right]={ }^{\prime} \widetilde{\Upsilon}_{\alpha \beta} \text {. }
$$

Considering frame transforms distortions to the canonical d-connection, ' $\widetilde{\mathbf{D}} \rightarrow{ }^{\prime} \widehat{\mathbf{D}}={ }^{\prime} \widetilde{\mathbf{D}}+\mathbf{Z}$, we prove

Corollary 4.4 (A general integrable canonical form of locally anisotropic Einstein equations on cotangent bundles) The modified Einstein equations (63) for Einstein-Hamilton phase spaces can be written equivalently in terms of the canonical d-connection ' $\widehat{\mathbf{D}}$, see Eq. (61), with re-defined sources, ${ }^{\prime} \widetilde{\Upsilon}_{\beta \gamma} \rightarrow{ }^{\prime} \widehat{\Upsilon}_{\beta \gamma}$.

We conclude that the gravitational field equations for the Einstein-Hamilton phase space geometry can be transformed into certain systems of nonlinear PDEs with decoupling properties. Examples of exact solutions are discussed in Directions 10-12, 18, 19 in Appendix B of [208]. Such solutions have been constructed by applying the AFDM for the case Einstein-Lagrange spaces and various modifications on nonholonomic manifolds. In dual form, the method can be developed for (modified) Einstein-Hamilton spaces.

Remark 4.3 ((Non)equivalence of Einstein-Lagrange and Einstein-Hamilton theories) The models of locally anisotropic gravity defined by equations (62) and (63) are different because they are derived on different phase spaces and for different types of d-metric and d-connection structures. Nevertheless, the geometric and physical data can be transformed equivalently from a tangent bundle to a cotangent bundle, and inversely, if a well-defined $L$-duality map (9) is considered.

Finally, we note that the locally anisotropic gravitational field equations (63) (with d-metric coefficients transformed into almost symplectic structures) can be studied in deformation quantization theories as in Refs. $[8,161]$. 


\subsection{MDRs and the Einstein-Yang-Mills-Higgs equations}

We can define such equations in abstract geometric form by extending the analogy Principle 3.4 both to Lagrange densities and gravitational and matter field equations on (co)tangent Lorentz bundles. All formulas can be derived alternatively applying the Principle-Convention 3.5 and Consequence 3.3 .

\subsubsection{EYMH systems on pseudo Lagrange spaces}

Modeling locally anisotropic gravitational and matter fields interactions on TV determined by geometric and physical data $\left(\mathbf{N}, \mathbf{g}, \mathbf{D} ; \mathbf{A}^{\check{a}}(u), \phi^{\check{a}}(u)\right)$ with a metric compatible d-connection D, we prove following geometric and/or $\mathrm{N}$ adapted variational methods:

Theorem 4.1 (MDR-modified EYMH equations on pseudo Lagrange phase spaces) Nonholonomic gravitational-scalargauge fields-Higgs field interactions on tangent Lorentz bundles can be described by such a system of nonlinear PDEs:

$$
\begin{aligned}
\mathbf{R}_{\alpha \beta}[\mathbf{D}] & ={ }^{\phi} \Upsilon_{\alpha \beta}+{ }^{A} \Upsilon_{\alpha \beta}+{ }^{H} \Upsilon_{\alpha \beta}, \\
\mathcal{D}_{\mu}\left(\sqrt{|\mathbf{g}|} \mathcal{F}^{\check{a} \mu \nu}\right) & =\frac{1}{2} i \check{e} \sqrt{|\mathbf{g}|}\left[\bar{\phi}^{\check{a}}, \mathcal{D}^{v} \phi^{\check{a}}\right], \\
\mathcal{D}^{\mu} \mathcal{D}_{\mu}\left(\sqrt{|\mathbf{g}|} \phi^{\check{a}}\right) & =\check{\lambda} \sqrt{|\mathbf{g}|} \mid\left(\left|\phi_{[0]}^{\check{a}}\right|^{2}-\bar{\phi}^{\check{a}} \phi^{\check{a}}\right) \phi^{\check{a}},
\end{aligned}
$$

with sources determined by the energy-momentum tensors (41), (45) and (46).

Equation (64) can be integrated in general off-diagonal forms for configurations with $\mathbf{D}=\widehat{\mathbf{D}}$ and, if necessary, for further restrictions to $\widehat{\mathbf{D}}_{\mid \widehat{\mathcal{T}}=0}=\nabla$. Exact classical and quantum solutions with generalized connections and for nonholonomic configurations have been studied in a series of works [143,144,146,152,153,156,157,161, $166,169,180,181]$. Recently, such solutions were found for locally anisotropic interactions of gravitational fields and effective matter fields, EYMH systems in GR and string gravity, for nonholonomic quantization of gauge models of gravity etc., see [23,190,198,203,209] and references therein. We note also that having constructed a class of solutions for $\widehat{\mathbf{D}}$ it is possible to define nonholonomic configurations with $\widetilde{\mathbf{D}}$ generating new classes of solutions via nonholonomic transforms and distorting nonlinear and linear connections. In such case, contributions of MDRs can be computed in explicit form.

\subsubsection{EYMH systems on pseudo Hamilton spaces}

On cotangent Lorentz bundles, we consider the data ( ' $\mathbf{N},{ }^{\prime} \mathbf{g}$, 'D; ' $\left.\mathbf{A}^{\check{a}}(u),{ }^{\prime} \phi^{\check{a}}\left({ }^{\prime} u\right)\right)$. Applying the analogy Principle 3.4, we formulate and prove
Theorem 4.2 (MDR-modified EYMH equations on pseudo Hamilton phase spaces) Nonholonomic gravitational-scalargauge fields-Higgs field interactions on cotangent Lorentz bundles are defined by a system of nonlinear PDEs with "dual” geometric data:

$$
\begin{aligned}
& { }^{\prime} \mathbf{R}_{\alpha \beta}\left[{ }^{\prime} \mathbf{D}\right]={ }_{1}^{\phi} \Upsilon_{\alpha \beta}+{ }_{1}^{A} \Upsilon_{\alpha \beta}+{ }_{1}^{H} \Upsilon_{\alpha \beta}, \\
& { }^{\prime} \mathcal{D}_{\mu}\left(\sqrt{\left|{ }^{\prime} \mathbf{g}\right|}{ }^{\prime} \mathcal{F}^{\check{a} \mu \nu}\right)=\frac{1}{2} i \check{e} \sqrt{\left|{ }^{\prime} \mathbf{g}\right|}\left[{ }^{\prime} \bar{\phi}^{\check{a}},{ }^{\prime} \mathcal{D}^{\nu}{ }^{\prime} \phi^{\check{a}}\right] \text {, } \\
& { }^{\prime} \mathcal{D}^{\mu}{ }^{\prime} \mathcal{D}_{\mu}\left(\sqrt{\left|{ }^{\prime} \mathbf{g}\right|}{ }^{\prime} \phi^{\check{a}}\right)=\check{\lambda} \sqrt{\left|{ }^{\prime} \mathbf{g}\right|}\left(\left.\left.\right|^{\prime} \phi_{[0]}^{\check{a}}\right|^{2}-\bar{\phi}^{\check{a}}{ }^{\prime} \phi^{\check{a}}\right){ }^{\prime} \phi^{\check{a}},
\end{aligned}
$$

with sources determined by the "dual" formulas for energymomentum tensors (41), (45) and (46). For simplicity, we can consider the same interaction constants for the $h-, v-$, and cv-subspaces.

The solutions for EYMH systems with MDRs on tangent Lorentz bundles and nonholonomic manifolds in GR and extra dimensions can be re-defined in cotangent bundle variables for $L$-dual (9) configurations when $\widehat{\mathbf{D}} \rightarrow$ ' $\widehat{\mathbf{D}}$ are correlated with respective nonholonomic transforms and distortions for $\widetilde{\mathbf{D}} \rightarrow$ ' $\widetilde{\mathbf{D}}$. The symmetries of systems (65) encode more general symplectomorphisms of d-connection structures (for Hamilton systems) which usually are not considered for Lagrange type phase space models.

\subsection{Massive and bi-metric MGTs as Lagrange-Hamilton geometries}

We speculated on geometric principles for formulating MGTs with $f$-deformations, massive gravitons, bi-metric structures and MDRs in Sect. 3.2.4. In this subsection, we provide main Theorems on such modifications of the Einstein equations on nonholonomic (co)tangent Lorentz bundles.

\subsubsection{Generalized Einstein equations with massive graviton on tangent Lorentz bundles}

Following a $\mathrm{N}$-adapted variational calculus for the action in Principle-Convention 3.6, we prove

Theorem 4.3 (MDR-modified massive bi-metric gravity on tangent Lorentz bundles) Nonholonomic $f$-modified and/or massive gravitational with bi-metric configurations on tangent Lorentz bundles are defined by such a system of nonlinear PDEs:

$\mathbf{R}_{\alpha \beta}[\mathbf{D}]={ }^{f \mu} \Upsilon_{\mu \nu}={ }^{g \mu} \Upsilon_{\mu \nu}+{ }^{f} \Upsilon_{\mu \nu}+{ }^{m} \Upsilon_{\mu \nu}$,

with sources determined by (50).

The equations (66) can be integrated following the AFDM for configurations with $\mathbf{D}=\widehat{\mathbf{D}}$ and nonholonomic constraints for extracting LC-configurations, $\widehat{\mathbf{D}}_{\mid \widehat{\mathcal{T}}=0}=\nabla$. A series of locally anisotropic wormhole and cosmological solutions 
were constructed and analyzed in Refs. [200-202,207]. The right side of (66) can be completed with additional sources EYMH systems and/or modifications from string gravity, for nonholonomic quantization of gauge models of gravity etc. like we generated cosmological and black hole like solutions in $[23,190,198,203,209]$ and references therein. Such generalizations can be defined following Assumption 3.1 when the contributions of gauge and Higgs fields can be approximated with respect to $\mathrm{N}$-adapted frames to certain effective cosmological constants. In general frame/coordinate systems, such solutions are generic off-diagonal and for generalized connections with coefficients depending, in principle, on all phase space coordinated. In order to compute contributions of MDRs, we have to re-define the physical equations and solutions for $\widetilde{\mathbf{D}}$-configurations.

\subsubsection{Massive gravitational equations on cotangent Lorentz bundles}

On cotangent Lorentz bundles, the main result for $f$-modified massive/bi-metric gravity is stated by

Theorem 4.4 (MDR-modified massive bi-metric gravity on cotangent Lorentz bundles) Nonholonomic $f$-modified and/or massive gravitational with bi-metric configurations on tangent Lorentz bundles are defined by such a system of nonlinear PDEs:

$$
{ }^{\prime} \mathbf{R}_{\alpha \beta}\left[{ }^{\prime} \mathbf{D}\right]={ }_{1}^{f \mu} \boldsymbol{\Upsilon}_{\mu \nu}={ }_{1}^{g \mu} \boldsymbol{\Upsilon}_{\mu \nu}+{ }_{1}^{f} \boldsymbol{\Upsilon}_{\mu \nu}+{ }_{1}^{m} \boldsymbol{\Upsilon}_{\mu \nu},
$$

with sources determined by (51).

Examples of exact solutions for the system (67) are discussed in Directions 10, 18, 19 in Appendix B of [208]. Such solutions exist at least as $L$-dual ones which have been constructed for (66).

\subsection{Short-range locally anisotropic gravity}

In this section, we formulate the gravitational filed equations for short-range gravitational toy models determined by MDRs with LIVs on (co)tangent Lorentz bundles with total phase spaces of $3+3$ dimension. Such models are constructed following the Principle-Convention 3.7.

\subsubsection{Field equations for short-range gravity with MDRs}

Following a N-adapted variational calculus of actions (52) on tangent bundle, we prove

Theorem 4.5 (Gravitational field equations for short-range gravity with MDR and LIV on tangent bundles) Nonholonomic short-range gravitational equations on tangent bun- dles determined by MDRs and LIVs can be written in the form

$\mathbf{R}_{\beta \delta}[\mathbf{D}]={ }^{s r} \boldsymbol{\Upsilon}_{\beta \delta}={ }^{m} \boldsymbol{\Upsilon}_{\beta \delta}+{ }^{l v} \boldsymbol{\Upsilon}_{\beta \delta}$,

with sources determined by energy-momentum d-tensors (53).

Examples of exact solutions for the system (68) for $\mathbf{D}=\widehat{\mathbf{D}}$ will be provided in our future partner works. Such solutions consist some particular 6-d examples (with short range effective source and associated cosmological constants, see Assumption 3.3) of 8-d and 10-d solutions constructed in Refs. [23, 68, 209].

\subsubsection{Field equations for short-range co-gravity with MDRs}

Following geometric methods (considering the dual of the short range gravity equations from tangent to cotangent bundles) and $\mathrm{N}$-adapted variational calculus of actions (52) on cotangent bundle, we prove

Theorem 4.6 (Gravitational field equations for short-range gravity with MDR and LIV on cotangent bundles) Nonholonomic short-range gravitational equations on cotangent bundles determined by MDRs and LIVs can be written in the form

$$
{ }^{\prime} \mathbf{R}_{\alpha \beta}\left[{ }^{\prime} \mathbf{D}\right]={ }_{1}^{f \mu} \boldsymbol{\Upsilon}_{\mu \nu}={ }_{1}^{g \mu} \boldsymbol{\Upsilon}_{\mu \nu}+{ }_{1}^{f} \boldsymbol{\Upsilon}_{\mu \nu}+{ }_{1}^{m} \boldsymbol{\Upsilon}_{\mu \nu},
$$

with sources determined by energy-momentum d-tensors (53) with labels " "”.

The systems (68) and/or (69) can be extended to 8-d and 10 -d phase spaces. Such black hole and cosmological type solutions (in general, with nontrivial torsion) in Directions 18 and 19 of Appendix B in [208]. Using nonholonomic frame transforms and distortions of d-connections, the short-range gravitational equations and respective exact/parametric solutions can be re-defined equivalently as Finsler-LagrangeHamilton systems of higher order. Geometrically, such generic off-diagonal equations are equivalent, or can be imbedded, into certain classes of physically important solutions constructed in $[23,190,198,203,209]$ and references therein.

\subsection{Towards axiomatic formulation of MGTs with MDRs on (co)tangent bundles}

A constructive-axiomatic approach to GR was proposed in 1964 by Ehlers et al. [43] (the system of so-called EPS axioms). It was completed with a series of conventions and results on physically important exact solutions and fundamental singularity and topological censorship theorems in gravity theories (for reviews, see monographs $[48,82,104$, 
213]). The concept of EPS spacetime, as a physically motivated model of spacetime geometry and locally isotropic gravitational interactions, was studied in a series of publications in the early 1970's, see original results and references in $[97,107,110,215]$. That EPS axiomatic approach led to an "orthodox" belief that the underlying spacetime geometry can be only pseudo-Riemannian. It resulted in the paradigmatic concept of "Lorentzian 4-manifold" described by a metric structure $g$ with pseudo-Euclidean signature $(+++-)$ and a corresponding unique metric compatible and torsionless (Levi Civita) LC-connection $\nabla$. It was postulated that the geometric data $(g, \nabla)$ are determined by a well-defined physical solution of the Einstein equations. For gravitational and matter field interactions, it was imposed a local causality condition that there are preserved locally all postulates of the special relativity theory, SRT.

All constructions in GR (on finite, or infinite, spacetime regions) are based on a well-defined physical principle of propagation of light along null geodesics. This way, a causal structure for massive particles and fields can be established if the speed of interactions is smaller than the maximal speed of light. The EPS axiomatic formalism is not valid, and has to be revised, for MGTs developed with the aim to explain acceleration and dark matter and dark energy in cosmology. Here we note that the ESP axiomatic approach has to be also extended in order to include in the scheme QG theories with MDRs and LIVs effects.

In this subsection, we conclude on how the axiomatic EPS scheme can be modified and generalized for Finsler like gravity theories. Such an approach was proposed in Refs. [187,194] for locally anisotropic spacetimes modeled on nonholonomic manifolds and vector/tangent bundles and can be performed in similar forms for MGTs with MDRs constructed on cotangent Lorentz bundles. In previous two sections, we have shown that the Lorentzian manifold geometric formulation of GR can be extended on TV and $\mathbf{T}^{*} \mathbf{V}$ for geometric data (35) defining models of generalized Einstein-Finsler/-Lagrange/-Hamilton gravity on phase spaces with MDRs and LIVs. We proved that using geometric and physical data for a large class of indicator functionals and respective canonical nonholonomic variables, $\varpi\left(x^{i}, E, \overrightarrow{\mathbf{p}}, m ; \ell_{P}\right) \rightarrow\left({ }^{\prime} \mathbf{g},{ }^{\prime} \mathbf{N}, \quad \widehat{\mathbf{D}}\right) \leftrightarrows(H:$ ' $\widetilde{\mathbf{g}}, \quad$ ' $\mathbf{N}, \quad \widetilde{\mathbf{D}}) \leftrightarrow\left[\left({ }^{\prime} \mathbf{g}\left[{ }^{\prime} N\right],{ }^{\prime} \nabla\right)\right]$ is possible to elaborate well-defined physical models in certain forms which are very similar to GR. In a more general context, the approach allows us to include in such a scheme various classes of MGTs with MDRs. There were considered models with multi-connection/-metric structure and motion equations formulated as effective Einstein-Yang-Mills-Higgs equations determined by equivalent geometric data $(\mathbf{g}, \mathbf{N}, \widehat{\mathbf{D}})$ and/or ( ' $\mathbf{g}$, ' $\mathbf{N}, ~ ' \widehat{\mathbf{D}})$. We argue that such gravitational and matter field equations possess certain generalized nonlinear symmetries with respect to redefinition of (effective) sources and distortion of distinguished (Finsler like) connections and fundamental geometric data.

Perhaps, the first attempt to formulate an axiomatic approach to Finsler gravity theories was considered in the early 80ths in the former URSS by Pimenov [109] in his habilitation thesis. In parallel, it was proposed also a minimal set of axioms for Finsler geometry due to M. Matsumoto, see a summary of work in [92]. Those works were not devoted to theories with multi-connection and multi-metric structures and had not studied how a corresponding Finsler axiomatic formalism should be related to the set of EPS axioms for GR. Recently, we proved [187,194] that the EPS approach can be generalized for a class of so-called Einstein-Finsler/Lagrange gravity theories. That geometric scheme was elaborated for nonholonomic deformations of fundamental geometric objects, $(g, \nabla) \rightarrow(\mathbf{g}, \mathbf{N}, \widehat{\mathbf{D}}) \leftrightarrows(L: \widetilde{\mathbf{g}}, \widetilde{\mathbf{N}}, \widehat{\mathbf{D}})$, working on nonholonomic manifolds, or tangent bundles, with $\mathrm{N}$-adapted splitting of total spacetime dimensions, $n+m=$ $2+1,2+2,2+2+1, \ldots$. Such locally anisotropic spacetimes (phase space gravity theories) are endowed with metrics of local pseudo-Euclidean signature and generalized Finsler connections, when probing point mass particles and light follow along semi-spray equations stating a causal structure at least for small nonholonomic deformations. Corresponding geometric methods and physical models were elaborated and studied in our monographs and articles on locally anisotropic black ellipsoid, wormhole, cosmological and other type solutions, see $[68,151,153,158,161,169,171,173,182,185,186$, $188,198,201,203]$ and references therein. Various issues on geometrization and axiomatic formulation of locally anisotropic relativistic/supersymmetric/noncommutative/ quantum deformed/string like/gauge like and other type theories (including corresponding types of MDRs) were studied in $[133,134,147,149,150,152]$, see recent results [23, 70, 117, 207,209].

A set of assumptions, conventions and principles which could be formalized into a complete system of axioms should be formulated for a generalized Finsler like theory (as a generalized Lagrange and/or Hamilton type) must be verified by experimental and observational data. This is a task for future research and theoretical constructions. In next subsection, we summarize key points and speculate only on structural blocks which have to be elaborated in detail for respective geometric models on tangent and/or cotangent Lorentz bundles:

\subsubsection{Assumptions on metrics, nonlinear quadratic line elements and d-metrics}

Let us discuss the assumptions considered in Sect. 2.1:

Assumption 2.1 (Background quadratic elements on total spaces of (co)tangent bundles) is important for theories with MDRs and LIVs which have a limit to a base Lorentz 
manifold with a typical Minkowski fiber for a corresponding tangent bundle. ${ }^{9}$ Using nonholonomic frame transforms and deformations of geometric structures, such background quadratic elements are transformed into general ones on (co)tangent Lorentz bundles. Intuitively, this assumption allows us to elaborate a causality structure on total phase space (at least for small nonholonomic deformations).

Assumption 2.2 (Nonlinear quadratic elements for modeling Finsler-Lagrange-Hamilton geometries on (co)tangent bundles) states a natural relation between the indicators of MDRs and respective generalized Finsler generating functions. For MDRs on cotangent Lorentz bundles, we can parameterize the construction in a form when the effective Hamiltonian can be considered as a generating function. Considering necessary type nonholonomic variables, we construct Legendre transforms to effective Lagrange generating functions. Relativistic and non-relativistic Finsler metrics can be considered for some particular cases when certain homogeneity conditions are imposed. It should be emphasized that such nonlinear quadratic line elements can be transformed into (pseudo) Riemannian ones only for very special "quadratic" configurations (as in the B. Riemann habilitation thesis [115]). We can consider some "simplified" examples of Randers type geometries, with applications in gravity and cosmology, see for instance, [15,61,63,127-129]. Nevertheless, a general assumption relating indicators with generating functions and nonlinear quadratic line elements is important for formulating an axiomatic approach to theories with MDRs.

Assumption 2.3 (d-metrics on (co)tangent Lorentz bundles) is motivated for physical theories geometrized with linear quadratic elements when nonlinear contributions are considered for certain small parameters, nonholonomic constraints and certain linearizable symmetries. We are not able to construct self-consistent and viable physically theories working only with nonlinear generating functions. Sasaki lifts of geometric objects from the base to total spaces (see details in monograph [218]) allows us to define a total metric structure completely defined by a generating (for instance, Finsler) function. More than that, we can perform such a construction in $\mathrm{N}$-adapted form and consider equivalent variants for nonholonomic Kaluza-Klein theories. In result, the concept of d-metric allows us to encode geometrically contributions for MDRs into generating functions and respective quadratic elements in frame/coordinate free forms.

Above assumptions should be completed with additional ones for Clifford bundles with $\mathrm{N}$-connections and spinor metric structures if MDRs are considered for spinor fields and generalized Dirac operators $[147,152,153,161,167,170$, 179].

\footnotetext{
${ }^{9}$ It is not obligatory defined in a smooth form and one could be considered nonholonomic constraints and various singular structures.
}

\subsubsection{Geometric and physical principles for Finsler-Lagrange-Hamilton gravity extensions}

Principles 3.1-3.3 formulated in Sect. 3.1 consist a set of main principles for extending GR to Finsler-LagrangeHamilton theories. We should add the Principle 3.4 if it is supposed that the locally anisotropic interactions of matter fields are modelled by Lagrange densities for a gravity theory with MDRs when the vacuum Finsler like gravitational fields are supposed to be described in a variational form (with minimal action).

Principle 3.1 (Modified equivalence principle) states that we can extend the fundamental equivalence principle for GR in self-consistent forms for certain classes of generalized Finsler theories. The constructions on (co)tangent Lorentz bundles are natural ones and allow "simplest causal" realizations of theories with MDRs. In such locally anisotropic spacetimes, free propagating particles and small perturbations of fields do not only follow certain (nonlinear) geodesic equations. They are described additionally by certain autoparallel equations which should be analyzed together with experiments and observational data for determining which linear connection is more appropriate for covariant derivatives. One can be constructed MGTs with multi-metric and multi-connection structures when a linear connection can be considered for one type of gravitationalmatter filed interactions and another type of linear connections taken for other types of matter field equations. Some metric structures can be considered as "hidden/un-physical" ones but one of them must be defined as the physical one. This allows us to define a Levi-Civita connection which should be completed with distorting tensors for respective linear connections and nonholonomic frame effects. A modified equivalence principle can be formulated and verified in a "minimal form" for metric compatible linear connections which are adapted to certain physically motivated $\mathrm{N}$-connection structures. In such a case, a causality structure can be established in a self-consistent form. It is not clear how such a principle could be formulated for general metric noncompatible connections. Nevertheless, if such a noncompatible connection is described by a unique distortion tensor from, for instance, the Levi-Civita connection, a modified equivalence principle can be reconsidered for certain congruences of special classes of curves determined by distortion relations.

Principle 3.2 (Generalized Mach principle) can be realized in more complex forms on generalized phase spaces modelled on (co)tangent Lorentz bundles. The geometric structure of such nonholonomic spaces is more richer and encodes more sophisticate correlations of spacetime properties and general locally anisotropic interactions with various noncommutative, stochastic, fractional configurations etc. In principle, we elaborate on certain models of generalized spacetime and 
phase space aether for different types of MGTs. The approach with nonholonomic bundles and manifolds allows us to formulate theories with MDRs and LIVs. For such theories, a generalized Mach principle can be verified by local and global observational and experimental data.

Principle 3.3 (General covariance and equivalent nonholonomic geometrizations of gravitational theories) states explicitly that the formulation of geometric models and physical theories should be performed in general frame and coordinate free forms. The nonholonomic and multi-connection structures of such locally anisotropic spacetime and phase spaces involve more sophisticated geometric constructions. If there are distortion relations for linear connections uniquely defined by the metric and $\mathrm{N}$-connection structure, all physically viable theories must admit an equivalent reformulation for any admissible and well-defined linear connection structure. Certain experimental/observational data may allow us choosing a physical connection for certain classes of matter filed interactions. Nevertheless, we shall be always able to distort in an equivalent form the physical data if a unique distortion connection structure can be defined. This is a consequence of the fact that theories with MDRs and LIVs are naturally geometrized on spaces with three fundamental geometric objects (N-connection, d-metric and d-connection) which is very different from (pseudo) Riemannian geometry determined completely by the metric structure.

Principle 3.4 (Analogy principle for Lagrange densities) has to be considered additionally to the previous ones if we attempt to construct variational physical models. For instance, we can substitute the LC-connection on a Lorentzian manifold by a metric compatible d-connection in the total space for all respective Lagrangians in GR. This way the constructions are naturally extended on (co)tangent Lorentz bundles and admit a self-consistent causal formulation.

Principle 3.4 has to re-formulated if spinor fields are introduced into consideration, see discussions for Direction 3 in Appendix B of [208].

\subsubsection{Conventions on Lagrangians and energy-momentum tensors on (co)tangent bundles}

The conventions, principles and assumptions formulated in Sect. 3.2 are necessary for constructing models with locally anisotropic gravitational and matter field interactions and MDRs in $\mathrm{N}$-adapted variational form. An explicit formulation of the main principles depends on the type of gravity and matter field theory we elaborate. For certain classes of canonical d-connections, such models can be elaborated to be exactly integrable in some general forms and/or quantized following methods of geometric/deformation quantization.

\section{On scalar field interactions and MDRs:}

Convention 3.1 (Scalar fields with MDRs on (co)tangent bundles) is for models of gravitational and matter field interactions with minimal coupling but extended on generalized phase spaces. In a similar form, we can postulate Lagrange densities with non-minimal coupling, complex scalar fields etc. when corresponding Lagrangians for MGTs on some manifolds are lifted by using Sasaki lifts with N-connections and d-metrics on total spaces of (co)tangent bundles.

\section{EYMH systems with MDRs on cotangent bundles:}

Convention 3.2 (Covariant derivatives of gauge fields in (co)tangent bundles) states that a metric compatible $\mathrm{d}$ connection on a phase space can be elongated by a gauge field potential for describing interactions with gauge fields in locally anisotropic spacetimes. In a simplest approach, we can consider canonical d-connections on (co)tangent bundles which allows us to integrate in some general forms respective gravitational - gauge filed equations with MDRs.

Convention 3.3 (Lagrange densities for YMH interactions on (co)tangent Lorentz bundles) is necessary for formulating Yang-Mills and Higgs theories with MDRs. The constructions are similar to those in GR but for respective metric compatible d-connection structures nonholonomically extended on total (co)bundle spaces.

Principle-Convention 3.5 (Actions for minimally MDRmodified EYMH systems) defines a model of locally anisotropic EYMH theory with minimal coupling on generalized Finsler-Lagrange-Hamilton spaces. MDRs and LIVs are encoded in respective generating functions via corresponding indicators. In a similar way, we can construct such theories to be with non-minimal coupling, for instance, in certain limits of (super) string theory.

Assumption 3.1 (Effective cosmological constants for locally anisotropic YMH sources) concerns both the possibility to construct self-dual gauge like configurations and to encode such configurations into an effective cosmological constant. For generalized Finsler spacetimes, this assumption allows us to consider a new class of nonlinear symmetries for generating functions, effective matter sources and effective cosmological constants.

\section{Massive gravity theories with MDRs:}

Principle-Convention 3.6 (Actions for MDR-modified massive gravity theories) is necessary for formulating massive gravity theories with MDRs and LIVs on (co)tangent Lorentz bundles. In particular, we can model a new class of locally anisotropic theories which similar GR on base spacetime but extended to effective massive gravity on phase space.

Assumption 3.2 (Effective cosmological constants in massive gravity on (co)tangent bundles) reflects the existence of a new 
class of nonlinear symmetries for generating functions and effective sources and cosmological constants.

\section{MDR-modified short-range gravity:}

Principle-Convention 3.7 (Lagrange densities for MDRmodified short-range gravity and LIV) is important for formulating a self-consistent extension of such short-range theories on (co)tangent bundles. This allows us to construct exact solutions in corresponding canonical variables by applying the AFDM.

Assumption 3.3 (effective cosmological constants for shortrange locally anisotropic interactions) is based on nonlinear symmetries of generating functions and effective matter fields and cosmological constants which can introduces for a large class of theories.

\section{Conservation laws (effective) sources and MDRs:}

The equations of motion and nonholonomic conservation laws for (effective) sources are discussed in Sect. 3.2.6. Additionally, we conclude:

Assumption 3.4 (energy-momentum d-tensors for (non) massive gravity theories on (co)tangent Lorentz bundles) allows us to define such values as in GR and MGTs on metricaffine manifolds but redefining the covariant derivatives into respective ones on (co)tangent bundles.

Principle 3.8 (nonholonomic deformations by MDRs of conservation laws on (co)tangent Lorentz bundles) reflects the properties that nonholonomic deformations and distortions result in theories for which the covariant derivative of the energy-momentum tensor is not zero. This is typical for nonholonomic systems, for instance, in nonholonomic mechanics when the variational problem is solved by using additional Lagrange multiples. In gravity theories constructed on nonholonomic manifolds and/or (co)tangent bundles, this issue is encoded into generalized Bianchi identities when distortions of covariant derivatives result in unique effective sources for nonzero covariant derivatives of energy momentum tensor. We can define distortions to LC-configurations resulting in effective sources which allows us to define conservation laws with covariant derivatives as in GR but on (co)tangent Lorentz bundles.

\subsubsection{Principles and main theorems for modified field equations with MDRs}

A series of principles stated in Sect. 4 can be formulated and derived as some main theorems which can be proven by respective $\mathrm{N}$-adapted variational calculus for respective actions and Lagrange densities outlined in previous subsection. Alternatively, such gravity theories can be formulated following only geometric principles like in the original A.
Einstein works, see details monograph [104]. For "pure" geometric constructions, the Principles-Theorems/-Corollaries and certain important Theorems discussed below can be considered as postulates for respective modified gravitational equations.

Principle-Theorem 4.1 (Nonholonomic modifications of Einstein equations on tangent Lorentz bundles) and PrincipleTheorem 4.2 (Nonholonomic modifications of Einstein equations on cotangent Lorentz bundles) can be formulated for the gravitational field equations on corresponding (co)tangent bundles. In general, such theories can be constructed in terms of triples (N-connection, d-connection and d-metric) without any assumptions on the properties of indicators of MDRs and/or related generating functions. The monographs $[153,161,169]$ and review [173] summarize such constructions for generalized Finsler and metric-affine spaces endowed with $\mathrm{N}$-connection structure.

Principle-Corollary 4.3 (generalized canonical Einstein equations for Lagrange gravity) and Principle-Corollary 4.4 (Generalized canonical Einstein equations for Hamilton gravity) are considered for canonical Lagrange and/or Hamilton variables which allow almost symplectic formulations. Such systems of nonlinear PDEs are equivalent if nonsingular Legendre transforms are defined. In general, such theories are not equivalent because they are described by different types of symmetries, different almost symplectic forms and symplectomorphisms, and non-equivalent dconnections. They have different classes of solutions and/or noncommutative/nonassociative and supersymmetric generalizations.

Theorem 4.1 (MDR-modified EYMH equations on pseudo Lagrange phase spaces) and Theorem 4.2 (MDR-modified EYMH equations on pseudo Hamilton phase spaces) show how we can formulate generalizations of EYMH theories with MDRs on pseudo Lagrange and/or Hamilton phase spaces. The constructions can be performed in canonical nonholonomic variables admitting decoupling and integration of fundamental field equations; using almost symplectic forms which is more suitable for deformation quantization; or in locally anisotropic spinor variables which allow twistor formulations and twistor quantization [143,153,161,169].

Theorem 4.3 (MDR-modified massive bi-metric gravity on tangent Lorentz bundles) and Theorem 4.4 (MDR-modified massive bi-metric gravity on cotangent Lorentz bundles) derive generalized Einstein equations for massive bi-metric gravity theories with MDRs on phase spaces. Such theories and exact solutions have been studied recently in a series of works $[24,68,76,113,114,123,135,200-202,207,210]$. In this paper, the gravitational field equations are proven originally for bi-metric and connection structures depending on momentum type coordinates. 
Theorem 4.5 (Gravitational field equations for short-range gravity with MDR and LIV on tangent bundles) and Theorem 4.6 (Gravitational field equations for short-range gravity with MDR and LIV on cotangent bundles) provide proofs how short-range gravity theories with MDRs [11] can be geometrized on (co)tangent Lorentz bundles.

Principles analyzed in this subsection have to be extended for new classes of nonholonomic distributions with $\mathrm{N}$ - and d-connections if spinor fields and twistors are introduced into consideration $[143,161,205]$. It is possible to elaborate a N-adapted Palatini formalism [104], quasi-classical extensions, noncommutative and nonassociative generalizations, deformation quantization, gauge like gravity theories etc., see Refs. [32-34,37,38,42, 146, 147, 152, 156, 157, 159, 162, $167,168,170,179,190,206]$.

\section{Conclusions, achievements and perspectives}

In this work, we have presented a unified geometric perspective on modified gravity theories, MGTs, with modified dispersion relations, MDRs, encoding Lorentz invariance violations, LIVs. We omitted technical aspects but attempted to formulate an axiomatic background and elaborate on new geometric methods, and discuss solutions to conceptual and fundamental problems in such ways we understand them. As this article is not intended to be a complete review of generalized Finsler geometries and applications we were able to mention only the sources which are most relevant to our research interests. We discussed the most important fundamental contributions of various international schools and individual researchers, provided main bibliographic sources but we should note that citations on possible applications are not comprehensive. This work also contains original results on geometric, variational, and axiomatic formulations of a series of relativistic models of Hamilton-Lagrange geometry and commutative MGTs elaborated on (co)tangent Lorentz bundles. We emphasize that relevant historical remarks being important for understanding the logic and motivations for developing such directions in modern geometry and physics are presented in Appendix B of [208] (see also there a summary of the main achievements and results, and a discussion of open problems and perspectives).

\subsection{On geometric models of MGTs with MDRs and generalized relativistic Finsler spaces}

As we have shown in Sect. 2, a large class of MGTs can be geometrized as models of relativistic Finsler-LagrangeHamilton spacetimes elaborated on nonholonomic (co) tangent Lorentz bundles/manifolds. The fundamental geometric objects (defined as d-metrics, N-connections, and dconnections) in such generalized phase spacetimes are with generic dependence on "velocity or momentum" type coordinates and determined by respective generating functions. The first examples of such nonlinear quadratic linear elements (i.e. Finsler metrics which can be modelled as some particular examples with homogeneous Lagrange generating functions (4), see also Example 2.4) was considered in the famous habilitation thesis (B. Riemann, 1854) [115]. That author emphasized that for simplicity his research was restricted only to geometries with linear quadratic elements which allowed an axiomatic formulation for Riemann geometry and, later, for pseudo-Riemannian (Lorentz spacetime) geometry.

The term Finsler geometry was introduced due to (E. Cartan, 1935), see [27] and references therein. That monograph was devoted to further developments of locally anisotropic geometric models proposed in (P. Finsler, 1918) [46]. E. Cartan contributions in elaborating the fundamentals of Finsler geometry were crucial. He understood that such a geometry is determined not only by a (nonlinear) quadratic line element with certain homogeneity conditions but that there are also two other important fundamental geometric objects. It was used in a coefficient form a new geometric object later called the N-connection (10) and introduced in Finsler geometry a metric compatible and N-adapted distinguished connection (the so-called Cartan d-connection). A series of fundamental books [27-29] with definition of bundle spaces, spinors, torsion fields and Riemann-Cartan geometry, Pfaff forms for systems of partial differential equations, PDEs, etc. were translated in many languages and had a substantial influence for thousands of researchers on developments of Finsler geometry by research schools in France, Germany, the USA, Czech Republic, Romania, Hungary, Poland, Russia, Japan, China, India, Greece, Iran, Egypt etc.

We mention here certain very important contributions to Finsler geometry due to (L. Berwald, 1926, 1941) [20,21]. He introduced new types of $\mathrm{N}$ - and d-connection structures completely determined by a semi-spray generating functions. His d-connection is not metric compatible but also admits a semi-spray geometric formulation in terms of certain nonlinear geodesic congruences. Here, we note that the concept of $\mathrm{N}$-connection is equivalent to that of (C. Ehresmann, 1955) [44]. A rigorous and original study of N-connections in Finsler geometry was performed due to (A. Kawaguchi, 1937, 1952) [72,73].

A very important concept for geometrizing theories with locally anisotropic interactions and/or MDRs is that of nonholonomic manifold. Such a space is defined as a real or complex (super) manifold with prescribed nonintegrable distributions, in particular, with N-connections, which are very important in Finsler geometry and generalizations. In certain sense, any geometric and/or physical model involve nonholonomic structures/constraints/frames and correspondingly adapted geometric objects and for- 
mulas. The approach was elaborated by (G. Vrănceanu 1931, 1957) [211,212], see references therein and [19] on relevant papers beginning 1926). ${ }^{10}$ We cite also an independent research and similar results due to ( $\mathrm{Z}$. Horak, 1927) [52]. The geometry of nonholonomic manifolds may encode and/or model via respective classes of distributions various complex/noncommutative/supersymmetric Finsler, Lagrange, Hamilton and other type nonholonomic spaces. Certain methods of the geometry of nonholonomic manifold (generalizing also various constructions in nonholonomic mechanics and classical and quantum field theories with nonintegrable constraints) were very important for elaborating the AFDM [153,161,169,173,185] for generating exact and parametric solutions in MGTs.

Then, a very important contribution in Finsler geometry was due to (S. -S. Chern, 1948) [41]. He introduced a new type of d-connection which is $\mathrm{N}$-adapted, with zero torsion, and coincides with the Levi-Civita connection on the base manifold. But the Chern d-connection is metric noncompatible on the total tangent bundle. Here we note that there is also a Chern connection for complex manifolds which is different from that in Finsler geometry. In certain sense, Finsler-Chern spaces consist examples of the Weyl geometry [214] which is defined, in general, with metric-affine noncompatible metric and linear connection structures. In a detailed form with various applications and noncommutative modifications, Finsler-/Lagrange-/Hamilton-affine geometric and physical theories were studied both on nonholonomic manifolds and (co)tangent bundles endowed with generalized Finsler like metric and N-connection structure, see monograph [169]. Chern's d-connection was re-discovered by Rund [118]. That monograph played a very important international role in the education of a new generation of researchers on Finsler geometry and applications. Later (after a number of Finsler books appeared in the former USSR, Romania and R. Moldova, due to G. Asanov, A. Bejancu, R. Miron and co-authors, S. Vacaru), it was published a series of important geometric books developing the Finsler-Chern geometry, see Bao et al. [16] and Shen [124] and references therein. Those three monographs, and mentioned above E.

\footnotetext{
${ }^{10}$ Historically, it is interesting to note that G. Vrănceanu was taught mathematics at the University of Iaşi (the first University in Romania) and studied at G öttingen under D. Hilbert; then at Rome under LeviCivita; and at Paris, where he worked with E. Cartan. His PhD was validated by an examining board consisted of 11 professors headed by V. Volterra. He was awarded a Rockefeller scholarship to study in France and the USA. In 1929, G. Vră nceanu was appointed as a professor at the Cernauti University which was one of the most important (at that time) in Romania, where he became one of the leading geometers (at that time) in the World. At present, his name is almost not known at the Chernivtsi State University, Ukraine. During his career as an university professor and leading member of the Romanian Academy of Sciences, Vrănceanu published more than 300 articles in all branches of modern geometry (till the end 1990).
}

Cartan and H. Rund books, are most cited and known books on Finsler geometry. Unfortunately, the style, methods and results of such works written by mathematicians, and respective geometric methods, are quite sophisticated for applications in modern physics and elaborating MGTs admitting limits to standard particle theories and GR. We had to elaborate our "metric compatible" approach to relativistic generalized Finsler theories, locally anisotropic gravity and cosmology as it was performed in $[8,12,153,161,161,169,172$ 174,177, 184, 185, 197,204,206].

Experts in geometry and physics know Chern's approach to Finsler geometry when the quadratic conditions are not imposed on linear quadratic elements and metric noncompatible d-connection structures are derived for nonlinear quadratic elements. Some authors consider that the Chern's d-connection is "the most appropriate one to study such locally anisotropic geometries and possible physical implications". ${ }^{11}$ We emphasize that it is a very difficult technical task and less physically motivated to elaborate well-defined Finsler-Chern/-Berwald (and other not metric compatible) modifications of standard physical theories. In brief, the main problems with applications in physics of such "noncompatible" geometries are those that the nonmetricity of a dconnection does not allow a self-consistent and generally accepted definition of spinors and Dirac operators and that formulation of some physically motivated conservative laws seems to be not possible. This creates a number of conceptual and technical difficulties for constructing physically viable, for instance, Finsler-Chern generalizations of GR; to formulate locally anisotropic (super) string and brane theories, noncommutative gravity and nonholonomic flow theories, EYMH and Einstein-Dirac system with MDRs; to study of locally anisotropic kinematic, stochastic and thermodynamic processes etc. Readers may consider critics of metric noncompatible physical models and alternative developments on Finsler MGTs in a series of works due to S. Vacaru and coauthors (almost all such works have been dubbed in arXiv.org and inspirehep.net or reviewed in MathSciNet and Zentralblatt $^{12}$ ). The main conclusions of those works were that there

\footnotetext{
11 For applications in physics and mechanics, it is more constructive to elaborate (generalized) Finsler geometry models defined in a selfconsistent physical and axiomatic mathematical form for certain fundamental metric, nonlinear and/or linear connection structures, vierbein and/or spinor like variables, etc. The N-adapted coefficients of such geometric objects are with generic dependence both on some basic spacetime coordinates and fiber (ed) type variables. We conclude that a Finsler space can be constructed as a nonholonomic geometric model with a nonlinear quadric line element, inducing certain fundamental metric and/or almost symplectic structures and generalized connections depending on velocity/momentum type coordinates on a (co)vector bundle or a nonholonomic fibered manifold. In explicit form, such FinslerLagrange-Hamilton theories can be elaborated for certain additional geometric and/or physical assumptions.

12 During 1987-1995, there were a series of original publications in the former USSR, the R. Moldova, and Romania. Here, we cite also some
} 
are natural extensions and/or equivalent realizations of GR and standard theories of particle physics, and various quantum models like loop quantum gravity/deformation quantization/noncommutative geometry etc. In a more general context, the constructions can be extended on (co)tangent Lorentz bundles enabled with d-metric $\mathrm{N}$-connection and Cartan d-connection (or other types metric compatible) structures determined by MDRs and respective fundamental generating Finsler-Lagrange-Hamilton functions. The fundamental field and/or evolution field equations in such theories can be transformed into systems of nonlinear PDEs with general decoupling property.

Some very influent schools on Finsler geometry, during 40 years after the second World War, were created in Japan. Such a research on pure and applied mathematics is related to two prominent geometers (A. Kawaguchi, 1937, 1952) [72,73] and (M. Matsumoto, 1966, 1986) $[17,91,92]$ and their coauthors. In this work, we emphasized the importance of a class of Finsler metric compatible geometric models which can be described in terms of almost Kähler variables, see Theorem 2.4 (they are important for deformation and geometric quantization $[8,12,161,172,174,177,184,197,204,206])$.

It should be noted that in Finsler geometry a symmetric variant of Ricci tensor can be constructed (H. Akbar Zadeh, 1988) $[1,2])$. The idea was to derive such a value directly from the fundamental generating function (Finsler metric) and semispray equations and do not enter in the ambiguities related to multi-connection character of such geometries. For applications in physics, such an approach remains incomplete because we can not elaborate viable theories without "covariant derivatives", i.e. without introducing a Finsler dconnection, on total bundle spaces (see discussions related to formula (38)).

\subsection{The physical picture of Finsler-Lagrange-Hamilton gravity theories}

The special relativity, SR, and general relativity, GR, theories have modified our understanding of causal and spacetemporal structure of classical reality in a way admitting an axiomatic formulation but whose consequences have not been fully explored yet. Various directions of research in QG and, for instance, modern accelerating cosmology with DE and DM problem result in fundamental problems on formulating theories with MDRs and possible LIVs. Our exploration of foundational issues for geometrizing MGTs with MDRs give rise to the conclusion that classical and quantum physical models can be described in a self-consistent axiomatic form on (co)tangent Lorentz bundles. This way

further original and review articles and books published by Western Editors beginning 1995 [153,161,169,173,185,193,194], see references therein and Appendix B in [208]. we have to revise a series of fundamental and conventional physical ideas and principles for locally anisotropic phase spaces and work with more rich geometric structures and advanced mathematical methods. We keep a lot from a credible physical intuition which is similar to that in standard theories of physics and do not need any drastic conceptual elaborations, for instance, on absolutely new and different Finsler causality and axiomatic approaches which cannot be elaborated, in principle, for general generating functions and indicators of MDRs. Nevertheless, various possible nonlinear symmetries/classifications/conservation laws of locally anisotropic and inhomogeneous interactions and modifications of standard theories determined by a (generalized) Finsler metric (nonlinear quadratic element) can be encoded into respective data for effective d-metrics, N-connections and d-connections. We also emphasize that our approach allows to analyze and compute explicit classical and quantum MDRs and LIVs effects, to find exact solutions and quantize in both perturbative and non-perturbative forms such MGTs.

The SR theory is based on the idea that the concepts of Newton space and independent time can be unified partially for physical models on a flat Minkowski spacetime as a consequence of experimentally verified constant speed of light and homogeneous and isotropic properties of light propagation. This allows a self-consistent formulation of relativistic mechanics together with Maxwell electrodynamics and such a unification can be realized with a respective Poincaré/Lorentz invariance both the fundamental flat spacetime, main physical equations and respective conservation laws. The physical meaning of GR is that the concept of curved spacetime, modelled as a Lorentz manifold, and the gravitational field are the same entity when the causality structure is determined in any point (for instance, along with a light geodesic) as in SR. If any MDRs of type (1) is considered (as a modification from QG, a generalized commutative or noncommutative theory etc.), the geometric constructions are naturally extended to a (co)tangent Lorentz bundle. For elaborating such approaches, it is always invoked the concept of a generalized spacetime aether (phase space, or locally anisotropic spacetime) with rich geometric structure depending both on space, time and velocity/momentum type coordinates.

In a locally anisotropic spacetime, the fundamental concepts of space and time of Newton, the Minkowski spacetime in SR with electromagnetic but without gravitational interactions, and the GR curved spacetime (the gravitational field, in our approach with zero MDR indicator) can be reinterpreted for respective configurations of locally anisotropic gravitational and matter field in a conventional phase space. Such a locally anisotropic picture implies also physical entities - particles, propagating dynamically and under nonholonomic constraints, and perturbations of locally anisotropic interacting real and effective fields - are not all immersed 
in a space moving in time or falling along curved pseudoRiemannian geodesics and/or causal curves. All they do not live only in a (generalized) spacetime. The dynamics and evolution of particles and field are mutually correlated (let say, live on one another) via a locally anisotropic aether for a nonholonomic phase space. It is a matter of convention which type of (generalized) Finsler-Lagrange-Hamilton, or their almost symplectic models, we use for modelling such geometries and physical theories. This approach presents a natural generalization of curved spacetime geometry for any MDRs and LIVs encoded in a nonlinear quadratic element $L(x, y)(4)$ and/or $H(x, p)(5)$. Such fundamental generating functions can be of any nature and with very general nonlinear symmetries. It is not possible to formulate a selfconsistent and viable causal-axiomatic approach in terms of general nonlinear quadratic elements (for instance, considering that they may describe certain nonlinear geodesics with space, time, or null-like interpretation). We can elaborate axiomatic approaches as in GR but for Finsler generalized metrics/connections/nonholonomic frames if we make one natural assumption: for a nontrivial indicator $\varpi$ of MDRs, the dynamics and evolution of particles and field is geometrized by respective nonholonomic structures on (co)tangent Lorentz bundles.

\subsection{What has been achieved in mathematical and theoretical physics with Finsler methods?}

The problem of formulating realistic Finsler MGTs and applications in classical and quantum physics and mechanics has many aspects. There is a great number of important ideas, results, and methods scattered in the literature. In this work, we have attempted to collect the most important and perspective mathematical and theoretical physics results and geometric methods and to present an overall perspective on classical and quantum theories with generic local anisotropy, MDRs and LIVs. We argue that such theories can be constructed on Lorentz manifolds with nonholonomic fibered structure and/or on (co)tangent Lorentz bundles enabled with nonlinear connection structure. The nonholonomic geometric scheme (with nonlinear quadratic elements and generalized (non)linear connections can be preserved for various noncommutative/supersymmetric/quantum/fractional etc. modifications and generalized symmetries when the Lorentz symmetries are restricted, modified, or generalized.

The strategy of almost 35 years research activity and author's points of view were both personal but also adapted to a temporary state of art evolution of main directions in modern mathematical and theoretical physics. The choice of subjects was determined by own interests and skills in geometry and physics but also oriented to solve important problems in modern gravity, non-standard particle physics and modified cosmology. He apologizes to colleagues in math- ematics and physics for what is missing and omitted from considerations (see Appendix B in [208] for a historical survey of achievements due to other authors, and references therein). So much is missing and the reason was author's intellectual and time limits and restrictions on lengths of this article: It is not a book and not a general or status review report but an attempt to formulate an axiomatic unification of main principles and results based on most important 20 directions of research in modern Finsler MGTs and applications.

Conventionally, main achievements on generalized Finsler geometry and applications in modern physics correlated to author's research activity, experience, and important publications can be structured into Seven Strategic Directions:

1. MGTs with MDRs and LIVs and Finsler-LagrangeHamilton geometry, with applications in modern cosmology and astrophysics

2. Geometric methods for constructing exact and parametric off-diagonal solutions in GR, MGTs and geometric flow theories

3. Geometric and almost Kähler deformation quantization, perturmative and nonperturbative quantization methods for models with nonlinear and/or nonholonomic dynamics and locally anisotropic field interactions

4. Nonholonomic Clifford structures, spinors and twistors, and Dirac operators for Lagrange-Hamilton and Riemann

-Finsler spaces and analogous/modified gravity

5. (Non) commutative/associative Finsler geometry and gravity related to locally anisotropic (super) string/ gravity theories

6. Kinetic, stochastic, fractional, and geometric and statistical thermodynamical processes with local anisotropy, classical and quantum gravity theories, quantum geometric informatics

7. Nonholonomic geometric flow evolution, modified Ricci solitons and field equations in supersymmetric, commutative and noncommutative, fractional (modified) gravity theories

In a more general context, including author's collaborations with scholars and young researchers from Western Countries, Romania and R. Moldova, mentioned above seven strategic directions are related to Twenty Main Research Directions outlined and discussed in Appendix $B$ of [208] (in general, there are considered there more than 100 sub-directions of research in modern Finsler-LagrangeHamilton geometry and applications). We present also a synopsis of related works published by Western Editors but also by less known (former) Editors in Eastern Europe. A part of such works were usually reviewed in MathSciNet and Zentralblatt, partially dubbed in arXv.org, but they are still un-known and (in many cases) not cited correctly in mod- 
ern literature of particle physics, gravity, and cosmology and astrophysics.

\subsection{What is missing in the scheme and further perspectives}

The main ambition of this work was to formulate a general axiomatic approach to Finsler-Lagrange-Hamilton MGTs. To elaborate and study of such classical and quantum theories is inevitable if we consider generic nonlinear interactions and evolution models with MDRs. This results in Finsler like generalizations of Einstein gravity, standard particle physics, astrophysics and cosmology theories. A number of papers involves research on locally anisotropic thermodynamics, statistics and kinetics, and quantum information theories with applications of a series of concepts and methods from quantum mechanics, QFT, and QG. The main aspects that are still missing or not sufficiently developed in our scheme are the following:

1. What type of Finsler like MGTs should we elaborate? MDRs arise naturally in various QG theories, for instance, non perturbative effects dominate at the Plank scale and yield to a discrete structure of spacetime. But we can also consider nonlinear interactions in classical physics with locally anisotropic stochastic and kinetic processes, nonholonomic dynamics and evolution models. For geometric modeling of such processes, MDRs and LIVs can be considered as certain effective ones, or as some fundamental relations imposed by experimental/observational data. Another important question is if Finsler like theories could solve fundamental problems of accelerating cosmology and DE and DM physics? Having a well-defined axiomatic of a theory (we proved that this is possible for very general assumptions of FinslerLagrange-Hamilton theories) is not enough for knowing how to extract physics from it and provide logical and experimental tests. What is missing is a systematic analysis and experimental data which would allow us to decide if theories with generic local anisotropy are generic quantum ones, and/or for certain locally anisotropic media (classical and/or quantum), or a respective Finsler MGT provides a well defined approach in modern cosmology.

2. Nonholonomic (co)tangent Lorentz bundles or other type modifications? As we shown, self-consistent axiomatic approaches with well-defined causal structure can be naturally elaborated by extending the main principles of ST and GR. But certain interesting geometric and physical models can be elaborated for restricted/deformed/duble etc. linear and nonlinear symmetries. What physical implications may have Finsler-Lagrange-Hamilton theories with "very radical" modifications of the Einstein/string gravity theory, quantum mechanics etc. and how to treat such new concepts of locally anisotropic spacetime?

3. Difference between Finsler-Lagrange-Hamilton and almost symplectic variables. In general, such variables are described by different nonholonomic structures. For certain well defined conditions such classical theories are equivalent. But noncommutative/nonassociative/ quantum/string/fractional/stochastic ...MGTs are positively elaborated differently for different classes of nonholonomic variables. Such theories are described by different Lagrangians/Hamiltonians with corresponding generalized topological and geometric structures/ symmetries. In principle, we have to elaborate rigorously on all 20 directions (and various sub-directions) outlined in Appendix B of [208] in order to distinguish the constructions on (co)tangent bundles, nonholonomic manifolds, with effective velocity/momentum variables etc.

4. Physically important solutions on (co)tangent bundles should be constructed explicitly and distinguished in velocity and momentum type variables, in particular, for Hamilton like configurations. Further partner works will be devoted to:

(a) Generalization of the AFDM for generating exact solutions with noncommutative/nonassociative/ supersymmetric and stochastic/fractional Hamilton like and entropic variables

(b) Exact and parametric black hole and cosmological solutions with modified dispersions in FinslerLagrange-Hamilton theories on (co)tangent Lorentz bundles

(c) Modified dispersion relations for Einstein-Dirac systems and pseudo Finsler-Hamilton geometry on (co)tangent Lorentz bundles

(d) Exact and parametric solutions for Einstein-YangMills-Higgs systems with modified dispersion and pseudo Finsler-Hamilton geometry

(e) Cosmological solutions in Hamilton variables and quantum effects

5. Quantization of Finsler-Lagrange-Hamilton MGTs. At present we have a quite well developed scheme of deformation quantization of such theories. For future research, it is very important to elaborate on

(a) Quantum (non)commutative gauge like LagrangeHamilton MGTs

(b) Geometric quantization of generalized Finsler gravity theory

(c) Loop Quantum Gravity, LQG, with MDRs.

(d) Perturbative and semiclassical limits of quantum Finsler-Lagrange-Hamilton theories

6. Quantum geometric informatics and computations and generalized entropic methods, entanglement etc. con- 
sist a series of recent developments in modern informatics, physics, and cosmology. Some series of works involve nonholonomic classical and quantum geometric methods, entropic dynamics, geometric thermodynamics etc. How such constructions should be generalized for Finsler-Lagrange-Hamilton variables?

Finally, we conclude that there are many problems that we have to understand and then solve in order to get credible, viable and physically important theories of locally anisotropic spacetime and gravitational and matter field interactions, nonholonomic evolution processes, quantization and cosmological applications. The author of this article hopes that some readers that have followed the formulated axiomatic scheme for Finsler-Lagrange-Hamilton MGTs will develop the research and new directions.

Acknowledgements During 2012-2017, authors research on modified Finsler gravity theories were supported by a project IDEI in Romania, PN-II-ID-PCE-2011-3-0256, and a series of senior research fellowships for CERN and DAAD. Recently, such results were presented at the IUCSS Workshop on "Finsler Geometry and Lorentz Violation", May 12-13, 2017, Indiana University, Bloomington, USA (organizer A. Kostelecký) and a seminar at CSU Fresno, USA (host D. Singleton). The authors of this work thanks all his co-authors (see bibliography at the end). They are grateful for support, important discussions and collaboration to C. Arcuş, H. E. Brandt (passed away), I. Bubuianu, G. Calcagni, C. Castro Perelman, H. Dehnen, E. Elizalde, F. Etayo, I. Gottlieb (passed away), A. P. Kouretsis, K. Lämmerzahl, N. Mavromatos, C. Mociuţchi (passed away), J. Moffat, S. Odintsov, V. Perlick, E. Peyghan, S. F. Ponomarenko, M. Sá nchez, P. Stavrinos, E. V. Veliev, M. Vişinescu and others.

Open Access This article is distributed under the terms of the Creative Commons Attribution 4.0 International License (http://creativecomm ons.org/licenses/by/4.0/), which permits unrestricted use, distribution, and reproduction in any medium, provided you give appropriate credit to the original author(s) and the source, provide a link to the Creative Commons license, and indicate if changes were made.

Funded by SCOAP ${ }^{3}$.

\section{A Two important corollaries for $\mathbf{N}$-adapted formulas}

We provide some examples and technical results, formulas and proofs which are similar to those used for elaborating the AFDM and applications to MGTs in Refs. $[68,151,153$, $158,161,169,171,173,182,186,188,198,201,203]$.

\section{A.1 Curvatures and torsions of d-connections on (co)tangent bundles}

By explicit computations for $\mathbf{X}=\mathbf{e}_{\alpha}, \mathbf{Y}=\mathbf{e}_{\beta}, \mathbf{D}=\left\{\boldsymbol{\Gamma}_{\alpha \beta}^{\gamma}\right\}$ and ${ }^{\prime} \mathbf{X}={ }^{\prime} \mathbf{e}_{\alpha},{ }^{\prime} \mathbf{Y}={ }^{\prime} \mathbf{e}_{\beta},{ }^{\prime} \mathbf{D}=\left\{{ }^{\prime} \boldsymbol{\Gamma}_{\alpha \beta}^{\gamma}\right\}$ in (29), we prove

Corollary A.1 For a d-connection $\mathbf{D}$ or ' $\mathbf{D}$, there are computed corresponding $\mathrm{N}$-adapted coefficients: d-curvature, $\mathcal{R}=\left\{\mathbf{R}_{\beta \gamma \delta}^{\alpha}=\left(R_{h j k}^{i}, R_{b j k}^{a}, P_{h j a}^{i}, P_{b j a}^{c}\right.\right.$,

$\left.\left.S_{h b a}^{i}, S_{b e a}^{c}\right)\right\}$, for

$R_{h j k}^{i}=\mathbf{e}_{k} L_{h j}^{i}-\mathbf{e}_{j} L_{h k}^{i}+L_{h j}^{m} L_{m k}^{i}-L_{h k}^{m} L_{m j}^{i}-C_{h a}^{i} \Omega_{k j}^{a}$,

$R_{b j k}^{a}=\mathbf{e}_{k} \dot{L}_{b j}^{a}-\mathbf{e}_{j} \dot{L}_{b k}^{a}+\dot{L}_{b j}^{c} \dot{L}_{c k}^{a}-\dot{L}_{b k}^{c} \dot{L}_{c j}^{a}-C_{b c}^{a} \Omega^{c}{ }_{k j}$,

$P_{j k a}^{i}=e_{a} L^{i}{ }_{j k}-D_{k} \hat{C}^{i}{ }_{j a}+\hat{C}^{i}{ }_{j b} T_{k a}^{b}$,

$P_{b k a}^{c}=e_{a} \dot{L}_{b k}^{c}-D_{k} C_{b a}^{c}+C_{b d}^{c} T_{k a}^{c}$,

$S^{i}{ }_{j b c}=e_{c} \dot{C}^{i}{ }_{j b}-e_{b} \dot{C}^{i}{ }_{j c}+\dot{C}^{h}{ }_{j b} \dot{C}^{i}{ }_{h c}-\dot{C}^{h}{ }_{j c} \dot{C}^{i}{ }_{h b}$,

$S_{b c d}^{a}=e_{d} C_{b c}^{a}-e_{c} C_{b d}^{a}+C_{b c}^{e} C_{e d}^{a}-C_{b d}^{e} C_{e c}^{a}$,

or ${ }^{\prime} \mathcal{R}=\left\{{ }^{\prime} \mathbf{R}_{\beta \gamma \delta}^{\alpha}=\left({ }^{\prime} R_{h j k}^{i},{ }^{\prime} R_{a j k}^{b},{ }^{\prime} P_{h j}^{i}{ }^{a},{ }^{\prime} P_{c j}^{b}{ }^{a},{ }^{\prime} S^{i}{ }_{h b a}\right.\right.$, ' $\left.\left.S_{\text {bea }}^{c}\right)\right\}$, for

$$
\begin{aligned}
& { }^{\prime} R_{h j k}^{i}={ }^{\prime} \mathbf{e}_{k}{ }^{\prime} L^{i}{ }_{h j}-{ }^{\prime} \mathbf{e}_{j}{ }^{\prime} L^{i}{ }_{h k}+{ }^{\prime} L^{m}{ }_{h j}{ }^{\prime} L^{i}{ }_{m k} \\
& \text { - ' } L_{h k}^{m}{ }^{\prime} L^{i}{ }_{m j}-{ }^{\prime} C^{i}{ }_{h}{ }^{\prime} \Omega_{a k j}, \\
& { }^{\prime} R_{a j k}^{b}={ }^{\prime} \mathbf{e}_{k}{ }^{\prime} \dot{L}_{a j}^{b}-{ }^{\prime} \mathbf{e}_{j}{ }^{\prime} L_{a k}^{b}+{ }^{\prime} \dot{L}_{c j}^{b}{ }^{\prime} \dot{L}_{a k}^{c} \\
& \text { - ' } L_{c k}^{b}{ }^{\prime} L_{a j}^{c}-{ }^{\prime} C_{a}^{b c}{ }^{\prime} \Omega_{c k j} \text {, } \\
& { }^{\prime} P_{j k}^{i}{ }^{a}={ }^{\prime} e^{a}{ }^{\prime} L^{i}{ }_{j k}-{ }^{\prime} D_{k}{ }^{\prime} \dot{C}^{i}{ }_{j}^{a}+{ }^{\prime} \dot{C}^{i}{ }_{j}^{b}{ }^{\prime} T_{b k}{ }^{a} \text {, } \\
& { }^{\prime} P_{c k}^{b a}={ }^{\prime} e^{a}{ }^{\prime} L_{c k}^{b}-{ }^{\prime} D_{k}{ }^{\prime} C_{c}^{b a}+{ }^{\prime} C_{b d}^{c}{ }^{\prime} T_{k a}^{c}, \\
& { }^{\prime} S^{i}{ }_{j}^{b c}={ }^{\prime} e^{c}{ }^{\prime} \dot{C}^{i}{ }_{j}^{b}-{ }^{\prime} e^{b}{ }^{\prime} \dot{C}^{i c}{ }_{j}{ }^{\prime}+{ }^{\prime} \dot{C}^{h}{ }_{j}{ }^{b}{ }^{\prime} \dot{C}^{i c}{ }_{h} \\
& -{ }^{\prime} C^{h}{ }_{j}^{c}{ }^{1} \dot{C}^{i b}{ }_{h} \\
& { }^{\prime} S_{a}^{b c d}={ }^{\prime} e^{d}{ }^{\prime} C_{a}^{b c}-{ }^{b} e^{c}{ }^{\prime} C_{a}^{b d}+{ }^{\prime} C_{a}^{b c}{ }^{\prime} C_{b}^{e d} \\
& -{ }^{\prime} C_{e}^{b d}{ }^{1} C_{a}^{e c} \text {; }
\end{aligned}
$$

d-torsion, $\mathcal{T}=\left\{\mathbf{T}_{\alpha \beta}^{\gamma}=\left(T^{i}{ }_{j k}, T_{j a}^{i}, T_{j i}^{a}, T_{b i}^{a}, T_{b c}^{a}\right)\right\}$, for

$$
\begin{aligned}
& T^{i}{ }_{j k}=L_{j k}^{i}-L_{k j}^{i}, T^{i}{ }_{j b}=C_{j b}^{i}, T^{a}{ }_{j i}=-\Omega^{a}{ }_{j i}, \\
& T_{a j}^{c}=L_{a j}^{c}-e_{a}\left(N_{j}^{c}\right), T_{b c}^{a}=C_{b c}^{a}-C_{c b}^{a} \text {, } \\
& \text { or }{ }^{\prime} \mathcal{T}=\left\{{ }^{\prime} \mathbf{T}_{\alpha \beta}^{\gamma}=\left({ }^{\prime} T_{j k}^{i},{ }^{\prime} T_{j}^{i}{ }_{j}^{a},{ }^{\prime} T_{a j i},{ }^{\prime} T_{a i}^{b},{ }^{b} T_{a}^{b c}\right)\right\}, \text { for } \\
& { }^{\prime} T^{i}{ }_{j k}={ }^{\prime} L_{j k}^{i}-{ }^{\prime} L_{k j}^{i},{ }^{\prime} T^{i}{ }_{j}^{a}={ }^{\prime} C_{j}^{i a},{ }^{\prime} T_{a j i}=-{ }^{\prime} \Omega_{a j i}, \\
& { }^{\prime} T_{c j}^{a}={ }^{\prime} L_{c j}^{a}-{ }^{\prime} e^{a}\left({ }^{\prime} N_{c j}\right),{ }^{\prime} T_{a}^{b c}={ }^{\prime} C_{a}^{b c}-{ }^{\prime} C_{a}^{c b} ;
\end{aligned}
$$

d-nonmetricity, $\mathcal{Q}=\left\{\mathbf{Q}_{\gamma \alpha \beta}=\left(Q_{k i j}, Q_{k a b}, Q_{c i j}, Q_{c a b}\right)\right\}$, for

$$
\begin{aligned}
& Q_{k i j}=D_{k} g_{i j}, Q_{k a b}=D_{k} g_{a b}, \\
& Q_{c i j}=D_{c} g_{i j}, Q_{c a b}=D_{c} g_{a b} \\
& o r{ }^{\prime} \mathcal{Q}=\left\{{ }^{\prime} \mathbf{Q}_{\gamma \alpha \beta}=\left({ }^{\prime} Q_{k i j},{ }^{\prime} Q_{k a b},{ }^{\prime} Q_{c i j},{ }^{\prime} Q_{c a b}\right)\right\}, \text { for } \\
& { }^{\prime} Q_{k i j}={ }^{\prime} D_{k}{ }^{\prime} g_{i j},{ }^{\prime} Q_{k}^{a b}={ }^{\prime} D_{k}{ }^{\prime} g^{a b}, \\
& { }^{\prime} Q^{c}{ }_{i j}={ }^{\prime} D^{c}{ }^{\prime} g_{i j},{ }^{\prime a b} Q^{c a b}={ }^{\prime} D^{c}{ }^{\prime} g^{a b} .
\end{aligned}
$$

A.2 The coefficients of canonical Lagrange and Hamilton d-connections

Such d-connections are very important for elaborating MGTs on (co)tangent bundles because they allow a very general 
decoupling and integration of generalized Einstein and matter field equations. By explicit computations in $\mathrm{N}$-adapted frames, we can prove that necessary conditions for defining and constructing, respectively, $\widehat{\mathbf{D}}$ (32) and ' $\widehat{\mathbf{D}}$ (33), are satisfied following

Corollary A.2 The N-adapted coefficients of canonical Lagrange and Hamilton d-connections are computed respectively:

$$
\begin{aligned}
\text { on TTV, } \widehat{\mathbf{D}}= & \left\{\widehat{\boldsymbol{\Gamma}}_{\alpha \beta}^{\gamma}=\left(\widehat{L}_{j k}^{i}, \widehat{L}_{b k}^{a}, \widehat{C}_{j c}^{i}, \widehat{C}_{b c}^{a}\right)\right\}, \text { where }, \\
& \text { for }\left[\mathbf{g}_{\alpha \beta}=\left(g_{j r}, g_{a b}\right), \mathbf{N}=\left\{N_{i}^{a}\right\}\right] \\
\simeq & {\left[\widetilde{\mathbf{g}}_{\alpha \beta}=\left(\widetilde{g}_{j r}, \widetilde{g}_{a b}\right), \widetilde{\mathbf{N}}_{i}^{a}=\widetilde{N}_{i}^{a}\right] } \\
\widehat{L}_{j k}^{i}= & \frac{1}{2} g^{i r}\left(\mathbf{e}_{k} g_{j r}+\mathbf{e}_{j} g_{k r}-\mathbf{e}_{r} g_{j k}\right), \\
\widehat{L}_{b k}^{a}= & e_{b}\left(N_{k}^{a}\right)+\frac{1}{2} g^{a c} \\
& \left(e_{k} g_{b c}-g_{d c} e_{b} N_{k}^{d}-g_{d b} e_{c} N_{k}^{d}\right) \\
\widehat{C}_{j c}^{i}= & \frac{1}{2} g^{i k} e_{c} g_{j k}, \\
\widehat{C}_{b c}^{a}= & \frac{1}{2} g^{a d}\left(e_{c} g_{b d}+e_{b} g_{c d}-e_{d} g_{b c}\right)
\end{aligned}
$$

and, on $T \mathbf{T}^{*} \mathbf{V}, \quad ' \widehat{\mathbf{D}}=\left\{{ }^{\prime} \widehat{\boldsymbol{\Gamma}}^{\gamma}{ }_{\alpha \beta}=\left({ }^{\prime} \widehat{L}_{j k}^{i},{ }^{\prime} \widehat{L}_{a k}^{b},{ }^{\prime} \widehat{C}^{i c}{ }_{j},{ }^{\prime} \widehat{C}^{i c}{ }_{j}\right)\right\}$, where,

$$
\begin{aligned}
& \text { for }\left[{ }^{\prime} \mathbf{g}_{\alpha \beta}=\left({ }^{\prime} g_{j r},{ }^{\prime} g^{a b}\right)\right. \text {, } \\
& \left.{ }^{\prime} \mathbf{N}=\left\{{ }^{\prime} N_{a i}\right\}\right] \\
& \simeq\left[{ }^{\prime} \widetilde{\mathbf{g}}_{\alpha \beta}=\left({ }^{\prime} \widetilde{g}_{j r},{ }^{\prime} \widetilde{g}^{a b}\right),{ }^{\prime} \widetilde{\mathbf{N}}_{a i}={ }^{\prime} \widetilde{N}_{a i}\right], \\
& \widehat{L}_{j k}^{i}=\frac{1}{2}{ }^{\prime} g^{i r}\left({ }^{\prime} \mathbf{e}_{k}{ }^{\prime} g_{j r}+{ }^{\prime} \mathbf{e}_{j}{ }^{\prime} g_{k r}-{ }^{\prime} \mathbf{e}_{r}{ }^{\prime} g_{j k}\right) \text {, } \\
& { }^{\prime} \widehat{L}_{a k}^{b}={ }^{\prime} e^{b}\left({ }^{\prime} N_{a k}\right) \\
& +\frac{1}{2}{ }^{\prime} g_{a c}\left({ }^{\prime} e_{k}{ }^{\prime} g^{b c}-{ }^{\prime} g^{d c}{ }^{\prime} e^{b}{ }^{\prime} N_{d k}\right. \\
& \left.-{ }^{\prime} g^{d b}{ }^{\prime} e^{c}{ }^{\prime} N_{d k}\right), \\
& { }^{\prime} \widehat{C}^{i c}=\frac{1}{2}{ }^{\prime} g^{i k}{ }^{\prime} e^{c}{ }^{\prime} g_{j k}, \\
& { }^{\prime} \widehat{C}_{a}^{b c}=\frac{1}{2}{ }^{\prime} g_{a d}\left({ }^{\prime} e^{c}{ }^{\prime} g^{b d}+{ }^{\prime} e^{b}{ }^{\prime} g^{c d}-{ }^{\prime} e^{d}{ }^{\prime} g^{b c}\right) .
\end{aligned}
$$

In a similar form, we can prove that all $\mathrm{N}$-adapted coefficient formulas necessary formulating and finding solutions of physically important field and evolution equations in theories with MDRs and LIVs.

\section{References}

1. H. Akbar-Zadeh, Sur les espaces de Finsler a courbures sectionnelles constants. Acad. R. Belg. Bull. Cl. Sci. 74, 281-322 (1988)

2. H. Akbar-Zadeh, Generalized Einstein manifolds. J. Geom. Phys. 17, 342-380 (1995)

3. G. Amelino-Camelia, J.R. Ellis, N.E. Mavromatos, D.V. Nanopoulos, Distance measurement and wave dispersion in a Liouville string approach to quantum gravity. IJMPA 12 607-624, arXiv: hep-th/9605211 (1997). arXiv:hep-th/9605211
4. G. Amelino-Camelia, J.R. Ellis, N.E. Mavromatos, D.V. Nanopoulos, S. Sarkar, Potential sensitivity of gamma-ray burster observations to wave dispersion in vacuo. Nature 393, 763-765 (1998). arXiv:astro-ph/9712103

5. G. Amelino-Camelia, Doubly special relativity. Nature 418, 34 35 (2002). arXiv:gr-qc/0207049

6. G. Amelino-Camelia, Relativity in space-times with short distance structure governed by an observer independent (Planckian) length scale. Int. J. Mod. Phys. D 11, 35-60 (2009)

7. G. Amelino-Camelia, L. Barcaroli, G. Gubitosi, S. Liberati, N. Loret, Realization of doubly special relativistic symmetries in Finsler geometries. Phys. Rev. D 90, 125030 (2014). arXiv: 1407.8143

8. M. Anastasiei, S. Vacaru, Fedosov quantization of LagrangeFinsler and Hamilton-Cartan spaces and Einstein gravity lifts on (co)tangent bundles. J. Math. Phys. 50, 013510 (2009). arXiv:0710.3079

9. S. Anco, S. Vacaru, Curve flows in Lagrange-Finsler geometry, bi-Hamiltonian structures and solitons. J. Geom. Phys. 59, 79-103 (2009). arXiv:math-ph/0609070

10. G.S. Asanov, Finsler Geometry, Relativity and Gauge Theories (Reidel, Dordrecht, 1985)

11. Q.G. Bailey, V.A. Kostelecky, X. Rui, Short-range gravity and Lorentz violation. PRD 91, 022006 (2015). arXiv:1410.6162

12. D. Baleanu, S. Vacaru, Fractional almost Kaehler-Lagrange geometry. Nonlinear Dyn. 64, 365-373 (2011). arXiv:1006.5535

13. D. Baleanu, S. Vacaru, Constant curvature coefficients and exact solutions in fractional gravity and geometric mechanics. Cent. Eur. J. Phys. 9, 1267-1279 (2011). arXiv:1007.2864

14. L. Barcaroli, L.K. Brunkhorst, G. Gubitosi, N. Loret, C. Pfeifer, Hamilton geometry: phase space geometry from modified dispersion relations. Phys. Rev. D 92, 084053 (2015). arXiv:1507.00922

15. S. Basilakos, A.P. Kouretsis, E.N. Saridakis, P. Stavrinos, Resembling dark energy and modified gravity with Finsler-Randers cosmology. PRD 88, 123510 (2013). arXiv:1311.5915

16. D. Bao, S.-S. Chern, Z. Shen, An Introduction to Riemann-Finsler Geometry. Graduate Text in Mathematics, vol. 200 (Springer, New York, 2000)

17. D. Bao, On two curvature-driven problems in Riemann-Finsler geometry, in Finsler Geometry. In Memory of M. Matsumoto. Proceedings of the 40th Symposium on Finsler Geometry, Sapporo, September 6-10, 2005. Advanced Studies in Pure Mathematics, vol. 48, ed. by S.V. Sabau et al. (Mathematical Society of Japan, Tokyo, 2007), pp. 19-71

18. A. Bejancu, Finsler Geometry and Applications (Ellis Horwood, Chichester, 1990)

19. A. Bejancu, H.R. Farran, Foliations and Geometric Structures (Springer, Berlin, 2003)

20. L. Berwald, Untersuchung der Krummung allgemeiner metrischer Raume auf Gunnd des in ihnen herrschenden Parllelismus. Math. Z. 25, 40-73 (1926)

21. L. Berwald, On Finsler and Cartan geometries, III. Twodimensional Finsler spaces with rectilinear extremals. Ann. Math. 42(2), 84-122 (1941)

22. D.G. Boulware, S. Deser, Can gravitation have a finite range? Phys. Rev. D 6, 3368-3382 (1972)

23. L. Bubuianu, K. Irwin, S. Vacaru, Heterotic supergravity with internal almost-Kaehler spaces; instantons for SO(32), or E8 x E8, gauge groups; and deformed black holes with soliton, quasiperiodic and/or pattern-forming structures. Class. Quantum Gravity 34, 075012 (2017). arXiv:1611.00223

24. Y.-F. Cai, F. Duplessis, E.N. Saridakis, F(R) nonlinear massive theories of gravity and their cosmological implications. Phys. Rev. D 90, 045004 (2014). arXiv:1307.7150

25. E. Caponio, G. Stancarone, Standard static Finsler spacetimes. IJGMMP 13, 1650040 (2016). arXiv:1506.07451 
26. S. Capozziello, V. Faraoni, Beyond Einstein gravity: a survey of gravitational theories for cosmology and astrophysics (Springer, Berlin, 2010)

27. E. Cartan, Les Espaces de Finsler (Herman, Paris, 1935)

28. E. Cartan, Lecons sur la theories des spineurs. Tome I: Les spineurs de l'espace a $\mathrm{n}>3$ dimensions. Les Spineurs en geometries riemanniene (Hermann, Paris, 1938); E. Cartan, The Theory of Spinors (Dover Publications, New York, 1966)

29. E. Cartan, La Methode du Repere Mobile, La Theorie des Groups Continus at les Espaces Generalises (Herman, Paris, 1963)

30. C. Castro, String theory, scale relativity and the generalized uncertainty principle. Found. Phys. Lett. 10, 273-293 (1997). arXiv:hep-th/9512044

31. C. Castro, The extended relativity theory in Born-Clifford phase spaces with a lower and upper length scales and Clifford group geometric unification. Found. Phys. 35, 971-1041 (2005)

32. C. Castro, On the noncommutative and nonassociative geometry of octonionic spacetime, modified dispersion relations and grand unification. J. Math. Phys. 48, 073517 (2007)

33. C. Castro, Born's reciprocal general relativity theory and complex nonabelian gravity as gauge theory of quaplectic group: a novel path to quantum gravity. IJMPA 23, 1487-1506 (2008)

34. C. Castro, On Born's deformed reciprocal complex gravitational theory and noncommutative gravity. Phys. Lett. B 668, 442-446 (2008)

35. C. Castro, Some consequences of Born's reciprocal relativity in phase-spaces. IJMPA 26, 3653-3678 (2011)

36. C. Castro, Gravity in curved phase-spaces, Finsler geometry and two-times physics. IJMPA 27, 1250069 (2012)

37. C. Castro, On Clifford space relativity, black hole entropy, rainbow metrics, generalized disperions and uncertainty relations. Found. Phys. 44, 990-1008 (2014)

38. C. Castro, A gauge theory of gravity in curved-phase-spaces. IJGMMP 13, 1650097 (2016)

39. Z. Chang, X. Li, Modified Newton's gravity in Finsler space as a possible alternative to dark matter hypothesis. Phys. Lett. B 668, 453-456 (2008). arXiv:0806.2184

40. Z. Chang, S. Wang, X. Li, Fine structure constant variation or spacetime anisotropy. EPJC 72, 1838 (2012). arXiv:1106.2726

41. S. Chern, Local equivalence and Euclidean connections in Finsler spaces. Sci. Rep. Nat. Tsing Hua Univ. Ser. A 5, 95-121 (1948); or Selected Papers, vol. II, 194 (Springer, 1989)

42. H. Dehnen, S. Vacaru, Nonlinear connections and nearly autoparallel maps in general relativity. Gen. Relativ. Gravit. 35, 807-850 (2003). arXiv:gr-qc/0009038

43. J. Ehlers, F.A. Pirani, A. Schild, The geometry of free fall and light propagation, in General Relativity. Papers Honour of J. L. Synge, ed. by L. O' Rifertaigh (Clarendon Press, Oxford, 1972), pp. 63-82

44. C. Ehresmann, Les connections infinitesimales dans un space fibre differentiable. Coloque de Topologie, Bruxelles. 1955, 29 55 (1955)

45. F. Etayo, R. Santamaría, S. Vacaru, Lagrange-Fedosov nonholonomic manifolds. J. Math. Phys. 46, 032901 (2005). arXiv:math.SG/0407495

46. P. Finsler, Ueber Kurven und Flaeschen in Allgemeiner Raemen. Dissertation, University of Gottingen 1918, Reprinted 1951 (Birkhauser, Basel, 1951)

47. S.F. Hassan, R.A. Rosen, Confirmation of the secondary constraint and absence of ghost in massive gravity and bimetric gravity. JHEP 1204, 123 (2012). arXiv: 1111.2070

48. S.W. Hawking, G.F.R. Ellis, The Large Scale Structure of the Space-Time. Cambridge Monographs on Mathematical Physics (1973)
49. M. Hohmann, Extensions of Lorentzian spacetime geometry: from Finsler to Cartan and vice versa. Phys. Rev. D 87, 123034 (2013). arXiv:1304.5430

50. M. Hohmann, Spacetime and observer space symmetries in the language of Cartan geometry. J. Math. Phys. 57, 082502 (2016). arXiv: 1505.07809

51. G. 't Hooft, Quantization of point particles in $(2+1)$-dimensional gravity and spacetime discreteness. CQG 13, 1023-1039 (1996)

52. Z. Horak, Sur les systemes non holonomes. Bull. Internt, Acad. Sci. Boheme. 1927, 1-18 (1927)

53. J.I. Horvath, A geometrical model for the unified theory of physical fields. Phys. Rev. 80, 901 (1950)

54. S. Ikeda, Some Finslerian features underlying the theory of physical fields. Lett. Nuovo Ciemnto (2) 15, 623-626 (1976)

55. S. Ikeda, Some field theoretical aspects underlying the theory of higher-order spaces. Lett. Nuveo Cimento (2) 20, 550-562 (1977)

56. S. Ikeda, A geometrical consideration on the concepts of unified gauge filed and supersymmetry. Lett. Nuovo Cimento 23, 455458 (1978)

57. S. Ikeda, On the theory of gravitational filed in Finsler spaces. Lett. Nuovo Cimento 26, 277-281 (1979)

58. S. Ikeda, On the conservation laws in the theory of fields in Finsler spaces. J. Math. Phys. 22, 1211-1214 (1981)

59. R.S. Ingarden, Ueber die Einbettung eines Finslerschen Rumes in einem Minkowskischen Raum (Regrman). Bull. Acad. Pol. Sci. Cl. III 2, 305-308 (1954)

60. R.S. Ingarden, J. Lawrynowicz, O. Suzuki, Superluminal signals and Finslerian physics. Bull. Soc. Sci. Lett. Lodz Ser. Rech. Deform. 39, 77-105 (2003)

61. R.S. Ingarden, Quantum Randers geometry as noncommuttive associative and commutative nonassociative geometries. Bull. Soc. Sci. Lett. Lodz Ser. Rech. Deform. 44, 7-22 (2004)

62. R.S. Ingarden, J. Lawrynowicz, Finsler geometry and physics. Mathematical overview. Bull. Soc. Sci. Lett. Lodz Ser. Rrech. Deform. 57, 51-56 (2008)

63. R.S. Ingarden, J. Lawrynowicz, Randers geometry and gauge theories. II. Randers and Kaluza-Klein gauge theories, self-duality and homogeneity. Bull. Soc. Sci. Lett. Lorz Ser. Rech. Deform. 56, 49-60 (2008)

64. H. Ishikawa, Einstein equations in lifted Finsler spaces. Nuovo Cimento B 56, 252-262 (1980)

65. H. Ishikawa, Note on Finslerian relativity. J. Math. Phys. 22, 995 (1981)

66. Y. Itin, C. Laemmerzahl, V. Perlick, Finsler-type modification of the Coulomb law. PRD 90, 124057 (2014). arXiv:1411.2670

67. M.A. Javaloyes, M. Sanchez, Finsler metrics and relativistic spacetimes. IJGMMP 11, 1460032 (2014). arXiv:1311.4770

68. T. Gheorghiu, O. Vacaru, S. Vacaru, Off-diagonal deformations of Kerr black holes in Einstein and modified massive gravity and higher dimensions. Eur. Phys. J. C 74, 3152 (2014). arXiv: 1312.4844

69. T. Gheorghiu, O. Vacaru, S. Vacaru, Modified dynamical supergravity breaking and off-diagonal super-Higgs effects. Class. Quantum Gravity 32, 065004 (2015). arXiv: 1311.6365

70. T. Gheorghiu, V. Ruchin, O. Vacaru, S. Vacaru, Geometric flows and Perelman's thermodynamics for black ellipsoids in R2 and Einstein gravity theories. Ann. Phys. NY 369, 1-35 (2016). arXiv: 1602.08512

71. F. Girelli, S. Liberati, L. Sindoni, Planck-scale modified dispersion relations and Finsler geometry. PRD 75, 0604015 (2006). arXiv:gr-qc0611024

72. A. Kawaguchi, Bezienhung zwischen einer metrischen linearen Ubertragung unde iener micht-metrischen in einem allmeinen metrishchen Raume. Akad. Wet. Amst. Proc. 40, 596-601 (1937) 
73. A. Kawaguchi, On the theory of non-linear connections, I. Tensor N. S. 2, 123-142 (1952)

74. A. Kawaguchi, On the theory of non-linear connections, II. Tensor N. S. 6, 165-199 (1956)

75. J. Kern, Lagrange geometry. Arch. Math. (Basel) 25, 438-443 (1974)

76. J. Klusoň, S. Nojiri, S.D. Odintsov, New proposal for nonlinear ghost-free massive $\mathrm{F}(\mathrm{R})$ gravity: cosmic acceleration and Hamiltonian analysis. Phys. Lett. B 726, 918-925 (2013). arXiv: 1309.2185

77. V.A. Kostelecky, Riemann-Finsler geometry and Lorentzviolating kinematics. Phys. Lett. B 701, 137-143 (2011). arXiv: 1104.5488

78. V.A. Kostelecky, N. Russel, R. Tso, Bipartite Riemann-Finsler geometry and Lorentz violation. Phys. Lett. B 716, 470 (2012). arXiv: 1209.0750

79. A.P. Kouretsis, M. Stathakopoulos, P.C. Stravrinos, Imperfect fluids, Lorentz violations and Finsler cosmology. Phys. Rev. D 82, 064035 (2010). arXiv: 1003.5640

80. A.P. Kouretsis, M. Stathakopoulos, P.C. Stavrinos, Relativistic Finsler geometry. Math. Methods Appl. Sci. 37, 223-229 (2014)

81. A.P. Kouretsis, Cosmic magnetization in curved and Lorentz violating space-times. EPJC 74, 2879 (2014). arXiv:1312.4631

82. D. Kramer, H. Stephani, E. Herdlt, M.A.H. MacCallum, Exact Solutions of Einstein's Field Equations, 2nd edn. (Cambridge University Press, Cambridge, 2003)

83. C. Laemmerzahl, D. Lorek, H. Dittus, Confronting Finsler spacetime with experiment. Gen. Relativ. Gravit. 41, 1345-1353 (2009). arXiv:0811.0282

84. C. Laemmerzahl, V. Perlick, W. Hasse, Observable effects in a class of spherically symmetric static Finsler spacetimes. PRD 86, 104042 (2012). arXiv: 1208.0619

85. X. Li, Z. Chang, Exact solution of vacuum field equation in Finsler spacetime. PRD 90, 064049 (2014). arXiv: 1401.6363

86. X. Li, S. Wang, Z. Chang, Finslerian perturbation for the $\Lambda$ CDM model. Commun. Theor. Phys. 61, 781-788 (2014). arXiv: 1309.1758

87. K. Lin, A spherical symmetrical spacetime solution in Finsler gravity. IJTP 53, 1271-1275 (2014)

88. X. Li, S. Wang, Primordial power spectrum of tensor perturbations in Finsler spacetime. EPJC 76, 51 (2016). arXiv:1508.04331

89. J. Lukierski, H. Ruegg, W.J. Zakrzewski, Classical quantum mechanics of free k-relativistic systems. Ann. Phys. 243, 90 (1995). arXiv:hep-th/9312153

90. J. Magueijo, L. Smolin, Gravity's rainbow. Class. Quantum Gravity 21, 1725 (2004). arXiv:gr-qc/0305055

91. M. Matsumoto, Connections, metrics and almost complex structures on tangent bundles. J. Math. Kyoto Univ. 5, 251-278 (1966)

92. M. Matsumoto, Foundations of Finsler Geometry and Special Finsler Spaces (Kaseisha Press, Tokyo, 1986)

93. N.E. Mavromatos, Decoherence and CPT violation in a stringy models of space-time foam. Found. Phys. 40, 917-960 (2010). arXiv:0906.2712

94. N.E. Mavromatos, String quantum gravity, Lorentz-invariance violation and gamma-ray astronomy. Int. J. Mod. Phys. A 25, 5409-5485 (2010). arXiv:1010.5354

95. N.E. Mavromatos, Quantum-gravity induced Lorentz violation and dynamical mass generation. Phys. Rev. D 83, 025018 (2011). arXiv: 1011.3528

96. N.E. Mavromatos, S. Sarkar, CPT-violating leptogenesis induced by gravitational defects. EPJC 73, 2359 (2013). arXiv:1211.0968

97. R. Meister, Eine Neuformulierung der EPS Axiomatik, Master's Thesis (Paderborn University, Paderborn, 1990)

98. E. Minguzzi, Light cones in Finsler spacetime. Commun. Math. Phys. 334, 1529-1551 (2015). arXiv:1403.7060
99. E. Minguzzi, Raychaudhuri equation and singularity theorems in Finsler spacetimes. CQG 32, 185008 (2015). arXiv:1502.02313

100. E. Minguzzi, An equivalence of Finslerian relativistic theories. Rep. Math. Phys. 77, 45-55 (2016). arXiv:1412.4228

101. R. Miron, M. Anastasiei, The Geometry of Lagrange Spaces: Theory and Applications. Fundamental Theories of Physics, vol. 59 (Kluwer Academic Publishers, Dordrecht, 1994)

102. R. Miron, D. Hrimiuc, H. Shimada, V.S. Sabau, The Geometry of Hamilton and Lagrange Spaces (Kluwer Academic Publishers, Dordrecht, 2000)

103. R. Miron, The Geometry of Higher-Order Hamilton Spaces (Kluwer Academic Publishers, Dordrecht, 2003)

104. C.W. Misner, K.S. Thorn, J.A. Wheeler, Gravitations (Freeman, Toronto, 1973)

105. S. Nojiri, S.D. Odintsov, Introduction to modified gravity and gravitational alternative for dark energy, eConf C 0602061 (2006). arXiv:hep-th/0601213 [Int. J. Geom. Methods Mod. Phys. 4, 115146 (2007)]

106. S. Nojiri, S.D. Odintsov, N. Shirai, Variety of cosmic acceleration models from massive $\mathrm{F}(\mathrm{R})$ bigravity. JCAP 1305, 020 (2013). arXiv: 1212.2079

107. V. Perlick, Characterization of standard clock by means of light rays and freely falling particles. Gen. Relativ. Gravit. 19, 10591073 (1987)

108. C. Pfeifer, M.N.R. Wohlarth, Finsler geometric extension of Einstein gravity. PRD 85, 064009 (2012). arXiv:1112.5641

109. R.I. Pimenov, Anisotropic Finsler Extension of General Relativity as an Order Structure (in Russian) (Fomi Filial AN SSSR, Syktyvkar, 1987)

110. F.A.E. Pirani, Building Space-Time from Light Rays and Free Particles. Symposia Mathematica, vol. XII (Academic Press, New York, 1973), pp. 67-83

111. F. Rahman, N. Paul, S.S. De, S. Ray, M.A.K. Jafry, The Finslerian compact star model. EPJC 75, 564 (2015). arXiv:1506.02501

112. F. Rahman, N. Paul, A. Banerjee, S.S. De, S. Ray, A.A. Usmani, The Finslerian wormhole models. EPJC 76, 246 (2016). arXiv: 1607.04329

113. C. de Rham, G. Gabadadze, Generalization of the Fierz-Pauli action. Phys. Rev. D 82, 044020 (2010). arXiv: 1007.0443

114. C. de Rham, G. Gabadadze, A.J. Tolley, Resummation of massive gravity. Phys. Rev. Lett. 106, 231101 (2011). arXiv:1011.1232

115. B. Riemann, Ueber die Hypothesen, welche der Geometries zugrunde liegen. Habilitationsvortrag 1854. Ges. Math. Werke (Leibzig, 1892), pp. 272-287. Reproduced (Dover Publications, 1953)

116. G. Rosati, G. Amelino-Camelia, A. Marciano, M. Matassa, Planck-scale-modified dispersion relations in FRW spacetime. Phys. Rev. D 92, 124042 (2015). arXiv:1507.02056

117. V. Ruchin, O. Vacaru, S. Vacaru, Perelman's W-entropy and statistical and relativistic thermodynamic description of gravitational fields. Eur. Phys. J. C 77, 184 (2017). arXiv:1312.2580

118. H. Rund, The Differential Geometry of Finsler Spaces (Springer, Berlin, 1959)

119. N. Russell, Finsler-like structures from Lorentz-breaking classical particles. PRD 91, 045008 (2015). arXiv:1501.02490

120. M. Schreck, Classical kinematics and Finsler structures for nonminimal Lorentz-violating fermions. EPJC 75, 187 (2015). arXiv: 1405.5518

121. M. Schreck, Eikonal approximation, Finsler structures, and implications for Lorentz-violating phonons in weak gravitational fields. PRD 92, 125032 (2015). arXiv: 1508.00216

122. M. Schreck, Classical Lagrangians and Finsler structures for the nonminimal fermion sector of the Standard-Model extension. PRD 93, 10517 (2016). arXiv: 1512.04299

123. L. Sebastiani, S. Vagnozzi, R. Myrzakulov, Mimetic gravity: a review of recent developments and applications to cosmology 
and astrophysics. Adv. High Energy Phys. 2017, 3156915 (2017). arXiv: 1612.08661

124. Z. Shen, Lectures on Finsler Geometry (World Scientific Publ. Co., Singapore, 2001)

125. Z. Shen, Differential Geometry of Spray and Finsler Spaces (Kluwer Academic Publishers, Dordrecht, 2001)

126. Z.K. Silagadze, On the Finslerian extension of the Schwarzschild metric. Acta. Phys. Pol. B 42, 1199-1206 (2011). arXiv:1007.4632

127. J.E.G. Silva, C.A.S. Almeida, Kinematics and dynamics in a bipartite-Finsler spacetime. Phys. Lett. B 731, 74-79 (2014). arXiv: 1312.7369

128. J.E.G. Silva, R.V. Maluf, C.A.S. Almeida, A nonlinear dynamics for the scalar field in Randers spacetime. Phys. Lett. B 766, $263-$ 267 (2017). arXiv: 1511.00769

129. J.E.G. Silva, Symmetries and fields in Randers-Finsler spacetime (preprint). arXiv:1602.07345

130. P. Stavrinos, S. Ikeda, Some connections and variational principle to the Finslerian scalar-tensor theory of gravitation. Rep. Math. Phys. 44, 221-230 (1999)

131. P.C. Stavrinos, F.I. Diakogiannis, Finslerian structure of anisotropic gravitational field. Gravit. Cosmol. 10, 269-278 (2004)

132. P.C. Stavrinos, Congruences of fluids in a Finslerian anisotropic space-time. Int. J. Theor. Phys. 44, 245-254 (2005)

133. P. Stavrinos, S. Vacaru, Cyclic and ekpyrotic universes in modified Finsler osculating gravity on tangent Lorentz bundles. Class. Quantum Gravity 30, 055012 (2013). arXiv: 1206.3998

134. P. Stavrinos, O. Vacaru, S. Vacaru, Off-diagonal solutions in modified Einstein and Finsler theories on tangent Lorentz bundles. Int. J. Mod. Phys. D 23, 1450094 (2014). arXiv:1401.2879

135. M. von Strauss, A. Schmidt-May, J. Enander, E. Mortsell, S.F. Hassan, Cosmological solutions in bimetric gravity and their observational tests. JCAP 1203, 042 (2012). arXiv:1111/1655

136. C. Tadmon, S. Plaise Tchapnada, On the spherically symmetric Einstein-Yang-Mills-Higgs equations in Bondi coordinates. Proc. R. Soc. Lond. A 468, 3191-3214 (2012). arXiv:1203.3291

137. Y. Takano, Theory of fields in Finsler spaces. I. Prog. Theor. Phys. 40, 1159-1180 (1968)

138. Y. Takano, Gravitational filed in Finsler spaces. Lett. Nuovo Cimento 10, 747-750 (1974)

139. R. Tavakol, Geometry of spacetime and Finsler geometry. IJMPA 24, 1678-1685 (2009)

140. A. Tayebi, E. Peyghan, A Kaehler structure on Finsler spaces with nonzero constant flag curvature. J. Math. Phys. 51, 022904 (2010)

141. A. Tayebi, E. Peyghan, On a class of Riemannian metrics arising from Finsler structures. C. R. Math. Acad. Sci. Paris 349, 319-322 (2011)

142. S.I. Vacaru, Multiphoton-graviton resonance processes and parametric amplification in gase like media, in Gravitational Waves, Works of the Conference on Problems on Radiation and Detection of Gravitational Waves, Joint Institute of Nuclear Researches, Dubna, URSS, 1983, D2, 13-83-689, ed. by A.F. Pisarev (JINR, Dubna, 1983), pp. 60-69 (in Russian)

143. S.I. Vacaru, Twistor-gauge interpretation of the Einstein-Hilbert equations. Vestnik Moscovskogo Universiteta. Phys. Astron. 28, 5-12 (1987) (in Russian). English translation: S.I. Vakaru, Mosc. Univ. Phys. Bull. 42, 1-7 (1987)

144. S. Vacaru, Nearly autoparallel maps and conservation laws on curved spaces. Rom. J. Phys. 39, 37-52 (1994)

145. S. Vacaru, S. Ostaf, Yu. Goncharenko, Nearly autoparallel maps and modeling of field interactions. Rom. J. Phys. 39, 199-212 (1994)

146. S. Vacaru, Yu. Goncharenko, Yang-Mills fields and gauge gravity on generalized Lagrange and Finsler spaces. Int. J. Theor. Phys. 34, 1955-1980 (1995)
147. S. Vacaru, Spinor structures and nonlinear connections in vector bundles, generalized Lagrange and Finsler spaces. J. Math. Phys. 37, 508-523 (1996)

148. S. Vacaru, Locally anisotropic stochastic processes in fiber bundles, in Proceeding of the Workshop "Global Analysis, Differential Geometry and Lie Algebras", December 16-18, 1995, Thessaloniki, Greece, ed. by G. Tsagas (Geometry Balkan Press, Bucharest, 1997), pp 123-140. http://www.mathem.pub.ro/proc/ bsgp-01/0VA.PDF. arXiv:gr-qc/9604014

149. S. Vacaru, Locally, anisotropic gravity and strings. Ann. Phys. (NY) 256, 39-61 (1997). arXiv:gr-qc/9604013

150. S. Vacaru, Superstrings in higher order extensions of Finsler superspaces. Nucl. Phys. B 434, 590-656 (1997). arXiv:hep-th/9611034

151. S. Vacaru, Exact solutions in locally anisotropic gravity and strings, in Particle, Fields and Gravitations. AIP Conference Proceedings, vol. 453, ed. by J. Rembielinski (American Institute of Physics, Woodbury, 1998), pp. 528-537. arXiv:gr-qc/9806080

152. S. Vacaru, Spinors and field interactions in higher order anisotropic spaces. JHEP 09(011), 1-49 (1998). arXiv:hep-th/9807214

153. S. Vacaru, Interactions, Strings and Isotopies in Higher Order Anisotropic Superspaces (Hadronic Press, Palm Harbor, 1998). arXiv:math-ph/0112056

154. S. Vacaru, Stochastic processes and thermodynamics on curved spaces. Ann. Phys. (Leipzig) 9(Special Issue), 175-176 (2000). arXiv:gr-qc/0001057

155. S. Vacaru, Locally anisotropic kinetic processes and thermodynamics in curved spaces. Ann. Phys. (N.Y.) 290, 83-123 (2001). arXiv:gr-qc/0001060

156. S. Vacaru, Gauge and Einstein gravity from non-Abelian gauge models on noncommutative spaces. Phys. Lett. B 498, 74-82 (2001). arXiv:hep-th/0009163

157. S.I. Vacaru, I.A. Chiosa, Nadejda A. Vicol, Locally anisotropic supergravity and gauge gravity on noncommutative spaces, in NATO Advanced Research Workshop Proceedings "Noncommutative Structures in Mathematics and Physics", September 23-27, Kyiv, Ukraine, ed. by S. Duplij, J. Wess (Kluwer Academic Publishers, Dordrecht, 2001), pp. 229- 243. arXiv:hep-th/0011221

158. S. Vacaru, Anholonomic soliton-dilaton and black hole solutions in general relativity. JHEP 04, 009 (2001). arXiv:gr-qc/0005025

159. S. Vacaru, F.C. Popa, Dirac spinor waves and solitons in anisotropic Taub-NUT spaces. Class. Quantum Gravity 18, 49214938 (2001). arXiv:hep-th/0105316

160. S. Vacaru, D. Singleton, V. Botan, D. Dotenco, Locally anisotropic wormholes and flux tubes in 5D gravity. Phys. Lett. B 519, 249258 (2001). arXiv:gr-qc/0103058

161. S. Vacaru, P. Stavrinos, Spinors and Space-Time Anisotropy (Athens University Press, Athens, 2002). arXiv:gr-qc/0112028

162. S. Vacaru, O. Tintareanu-Mircea, Anholonomic frames, generalized Killing equations, and anisotropic Taub NUT spinning spaces. Nucl. Phys. B 626, 239-264 (2002). arXiv:hep-th/0104075

163. S. Vacaru, D. Singleton, Ellipsoidal, cylindrical, bipolar and toroidal wormholes in 5D gravity. J. Math. Phys. 43, 2486-2504 (2002). arXiv:hep-th/0110272

164. S. Vacaru, Horizons and geodesics of black ellipsoids. Int. J. Mod. Phys. D. 12, 479-494 (2003). arXiv:gr-qc/0206014

165. S. Vacaru, Perturbations and stability of black ellipsoids. Int. J. Mod. Phys. D 12, 461-478 (2003). arXiv:gr-qc/0206016

166. S. Vacaru, H. Dehnen, Locally anisotropic structures and nonlinear connections in Einstein and gauge gravity. Gen. Relativ. Gravit. 35, 209-250 (2003). arXiv:gr-qc/0009039

167. S. Vacaru, N. Vicol, Nonlinear connections and spinor geometry. Int. J. Math. Math. Sci. 23, 1189-1237 (2004). arXiv:math.DG/0406585 
168. S. Vacaru, Exact solutions with noncommutative symmetries in Einstein and gauge gravity. J. Math. Phys. 46, 042503 (2005). arXiv:gr-qc/0307103

169. Clifford and Riemann-Finsler Structures in Geometric Mechanics and Gravity, Selected Works, by S. Vacaru, P. Stavrinos, E. Gaburov, D. Gonta, Differential Geometry—Dynamical Systems, Monograph 7 (Geometry Balkan Press, Bucharest, 2006). http:// www.mathem.pub.ro/dgds/mono/va-t.pdf. arXiv:gr-qc/0508023

170. S. Vacaru, Clifford-Finsler algebroids and nonholonomic Einstein-Dirac structures. J. Math. Phys. 47, 093504 (2006). arXiv:hep-th/0501217

171. S. Vacaru, Parametric nonholonomic frame transforms and exact solutions in gravity. Int. J. Geom. Methods Mod. Phys. 4, 12851334 (2007). arXiv:0704.3986

172. S. Vacaru, Deformation quantization of almost Kaehler models and Lagrange-Finsler spaces. J. Math. Phys. 48, 123509 (2007). arXiv:0707.1519

173. S. Vacaru, Finsler-Lagrange geometries and standard theories in physics: new methods in Einstein and string gravity. (a short variant was published in Int. J. Geom. Methods Mod. Phys. 5, 473511 (2008). See arXiv:0801.4958 [gr-qc]) and arXiv:0707.1524 [gr-qc]

174. S. Vacaru, Deformation quantization of nonholonomic almost Kaehler models and Einstein gravity. Phys. Lett. A 372, 29492955 (2008). arXiv:0707.1667

175. S. Vacaru, Finsler and Lagrange geometries in Einstein and string gravity. Int. J. Geom. Methods Mod. Phys. 5, 473-511 (2008). arXiv:0801.4958

176. S. Vacaru, Loop quantum gravity in Ashtekar and LagrangeFinsler variables and Fedosov quantization of general relativity. (Icfai Univ. J. Phys.) IUP J. Phys. II(4), 15-58 (2009). arXiv:0801.4942

177. S. Vacaru, Branes and quantization for an A-model complexification of Einstein gravity in almost Kaehler variables. Int. J. Geom. Methods Mod. Phys. 6, 873-909 (2009). arXiv:0810.4692

178. S. Vacaru, The entropy of Lagrange-Finsler spaces and Ricci flows. Rep. Math. Phys. 63, 95-110 (2009). arXiv:math.DG/0701621

179. S. Vacaru, Spectral functionals, nonholonomic Dirac operators, and noncommutative Ricci flows. J. Math. Phys. 50, 073503 (2009). arXiv:0806.3814

180. S. Vacaru, Nonholonomic distributions and gauge models of Einstein gravity. Int. J. Geom. Methods Mod. Phys. 7, 215-246 (2010). arXiv:0902.0911

181. S. Vacaru, Two-connection renormalization and nonholonomic gauge models of Einstein gravity. Int. J. Geom. Methods Mod. Phys. 7, 713-744 (2010). arXiv:0902.0961

182. S. Vacaru, On general solutions in Einstein and high dimensional gravity. Int. J. Theor. Phys. 49, 884-913 (2010). arXiv:0909.3949v4

183. S. Vacaru, Finsler black holes induced by noncommutative anholonomic distributions in Einstein gravity. Class. Quantum Gravity 27, 105003 (2010). arXiv:0907.4278

184. S. Vacaru, Einstein gravity as a nonholonomic almost Kaehler geometry, Lagrange-Finsler variables, and deformation quantization. J. Geom. Phys. 60, 1289-1305 (2010). arXiv:0709.3609

185. S. Vacaru, Critical remarks on Finsler modifications of gravity and cosmology by Zhe Chang and Xin Li. Phys. Lett. B 690, 224-228 (2010). arXiv:1003.0044v2

186. S. Vacaru, New classes of off-diagonal cosmological solutions in Einstein gravity. Int. J. Theor. Phys. 49, 2753-2776 (2010). arXiv: $1003.0043 \mathrm{v} 1$

187. S. Vacaru, Principles of Einstein-Finsler gravity and cosmology. J. Phys. Conf. Ser. 314, 012069 (2011). arXiv:1012.4148 [Proceedings of Spanish Relativity Meeting (ERE 2010): Gravity as a Crossroad in Physics, Granada, Spain, 6-10 September, 2010]
188. S. Vacaru, On general solutions in Einstein gravity. Int. J. Geom. Methods Mod. Phys. 8, 9-21 (2011). arXiv:0909.3949v1

189. S. Vacaru, Finsler branes and quantum gravity phenomenology with Lorentz symmetry violations. Class. Quantum Gravity 28, 215991 (2011). arXiv:1008.4912

190. S. Vacaru, Covariant renormalizable anisotropic theories and offdiagonal Einstein-Yang-Mills-Higgs solutions. Europhys. Lett. (EPL) 96, 50001 (2011). arXiv:1108.2023

191. S. Vacaru, Fractional nonholonomic Ricci flows. Chaos Solitons Fractals 45, 1266-1276 (2012). arXiv:1004.0625

192. S. Vacaru, Modified dispersion relations in Horava-Lifshitz gravity and Finsler brane models. Gen. Relat. Gravit. 44, 1015-1042 (2012). arXiv:1010.5457

193. S. Vacaru, Metric compatible or noncompatible Finsler-Ricci flows. Int. J. Geom. Methods Mod. Phys. 9, 1250041 (2012). arXiv: 1106.4888

194. S. Vacaru, Principles of Einstein-Finsler gravity and perspectives in modern cosmology. Int. J. Mod. Phys. D 21, 1250072 (2012) arXiv: 1004.3007

195. S. Vacaru, Diffusion and self-organized criticality in Ricci flow evolution of Einstein and Finsler spaces. Symmetry Cult. Sci. 23(nr. 2), 105-124 (2013). ISSN:0865-4824 (printed), ISSN:2226-1877 (online), Thematic Issue: Field theories on Finsler Space (Symmetries with Finsler metric, 2013). arXiv: 1010.2021

196. S. Vacaru, Black holes, ellipsoids, and nonlinear waves in pseudoFinsler spaces and Einstein gravity. Int. J. Theor. Phys. 52, 1654 1681 (2013). arXiv:0905.4401

197. S. Vacaru, The algebraic index theorem and Fedosov quantization of Lagrange-Finsler and Einstein spaces. J. Math. Phys. 54, 073511 (2013). arXiv:1005.3647

198. S. Vacaru, Decoupling of field equations in Einstein and modified gravity. J. Phys. Conf. Ser. 543, 012021 (2013). arXiv: $1108.2022 \mathrm{v} 3$

199. S. Vacaru, Wormholes and off-diagonal solutions in $\mathrm{f}(\mathrm{R}, \mathrm{T})$, Einstein and Finsler gravity theories, in Progress in Mathematical Relativity, Gravitation and Cosmology, Springer Proceedings in Mathematics \& Statistics, vol. 60, ed. by A. Garcia-Parrado, F.C. Mena, F. Moura. E. Vaz (2014), pp. 439-443. arXiv:1303.3515v1 [Proceedings of the Spanish Relativity Meeting ERE2012, University of Minho, Guimaraes, Portugal, September 3-7, 2012]

200. S. Vacaru, Covariant renormalizable modified and massive gravity theories on (non) commutative tangent Lorentz bundles. Int. J. Geom. Methods Mod. Phys. 11, 1450032 (2014). arXiv: 1304.1079

201. S. Vacaru, Exact solutions in modified massive gravity and offdiagonal wormhole deformations. Eur. Phys. J. C 74, 2781 (2014). arXiv: 1403.1815

202. S. Vacaru, Ghost-free massive $f(R)$ theories modelled as effective Einstein spaces \& cosmic acceleration. Eur. Phys. J. C 74, 3132 (2014). arXiv:1401.2882

203. S. Vacaru, E. Veli Veliev, E. Yazici, A geometric method of constructing exact solutions in modified $\mathrm{f}(\mathrm{R}, \mathrm{T})$ gravity with YangMills and Higgs interactions. Int. J. Geom. Methods Mod. Phys. 11, 1450088 (2014). arXiv: 1411.2849

204. S. Vacaru, Almost Kaehler Ricci flows and Einstein and Lagrange-Finsler structures on Lie algebroids. Mediterr. J. Math. 12, 1397-1427 (2015). arXiv:1306.2813

205. S. Vacaru, Spinors and twistor geometry in Einstein gravity and Finsler modifications. Adv. Appl. Clifford Algebras 25, 453-485 (2015). arXiv: 1206.4012

206. S. Vacaru, Noncommutative Einstein, almost Kaehler-Finsler and Quantum Deformations. Chapter 24, in The Algebraic Way. Space, Time and Quantum Beyond Peaceful Coexistence, ed. by Ignazio Licata (World Scientific, Singapore, 2016), pp. 661-695 
207. S. Vacaru, Off-diagonal ekpyrotic scenarios and equivalence of modified, massive and/or Einstein gravity. Phys. Lett. B 752, 27 33 (2016). arXiv: 1304.1080

208. S. Vacaru, On axiomatic formulations of gravity and matter field theories with MDRs and Finsler-Lagrange-Hamilton geometry on (co) tangent Lorentz bundles (preprint). arXiv:1801.06444

209. S. Vacaru, K. Irwin, Off-diagonal deformations of Kerr metrics and black ellipsoids in heterotic supergravity. Eur. Phys. J. C 77, 17 (2017). arXiv: 1608.01980

210. M.S. Volkov, Cosmological solutions with massive gravitons in the bigravity theory. JHEP 1201, 035 (2012). arXiv:1110.6153

211. G. Vrănceanu, Sur quelques point de la theories des espaces nonholonomies. Bull. Fac. Şt. Cernăuţi 5, 177-205 (1931)

212. G. Vrănceanu, Leçons de Geometrie Differentielle, vol. II (Edition de l'Academie de la Republique Populaire de la Roumanie, 1957)
213. R.W. Wald, General Relativity (University of Chicago Press, Chicago, 1984)

214. H. Weyl, Elektron und gravitation. Z. Phys. 56, 330 (1929)

215. N.M.J. Woodhouse, The differentiable and causal structures of space-time. J. Math. Phys. 14, 495-501 (1973)

216. H.S. Yang, Emergent gravity from noncommutative spacetime. IJMPA 24, 4473-4517 (2009). arXiv:hep-th/0611174

217. Hyun Seok Yang, M. Sivakumar, Emergent gravity from quantized spacetime. Phys. Rev. D 82, 045004 (2010). arXiv:0908.2809

218. K. Yano, S.I. Ishihara, Tangent and Cotangent Bundles. Differential Geometry (Marcel Dekker, New York, 1973)

219. N.L. Youssef, S.H. Abed, A. Soleiman, A global approach to the theory of connections in Finsler geometry. Tensor N. S. 71, 187208 (2009). arXiv:0801.3220 